\title{
AC-Conductivity Measure from Heat Production of Free Fermions in Disordered Media
}

\author{
J.-B. Bru \\ W. de Siqueira Pedra \\ C. Kurig
}

October 28, 2013

\begin{abstract}
We extend [BPK2] in order to study the linear response of free fermions on the lattice within a (independently and identically distributed) random potential to a macroscopic electric field that is time- and space-dependent. We obtain the notion of a macroscopic AC-conductivity measure which only results from the second principle of thermodynamics. The latter corresponds here to the positivity of the heat production for cyclic processes on equilibrium states. Its Fourier transform is a continuous bounded function which is naturally called (macroscopic) conductivity. We additionally derive GreenKubo relations involving time-correlations of bosonic fields coming from current fluctuations in the system. This is reminiscent of non-commutative central limit theorems.
\end{abstract}

\section{Contents}

1 Introduction 2

2 Setup of the Problem 6

2.1 Anderson Tight-Binding Model . . . . . . . . . . . . . . 6

2.2 Coupling to Electromagnetic Fields . . . . . . . . . . . . . 7

2.3 Algebraic Approach . . . . . . . . . . . . . . . 10 
3 CCR Algebra of Fluctuations of Ohm's Law 12

3.1 Macroscopic Transport Coefficients . . . . . . . . . . . . 12

3.2 Classical Ohm's Law . . . . . . . . . . . . . . . . . . . . . 16

3.3 Green-Kubo Relations and CCR Algebra of Current Fluctuations 19

4 AC-Conductivity Measure From Joule's Law 22

4.1 Energy Densities . . . . . . . . . . . . . . . . . 23

4.2 Classical Joule's Law . . . . . . . . . . . . . . . . . . . 26

4.3 AC-Conductivity Measure . . . . . . . . . . . . . . 29

5 Technical Proofs 33

5.1 Complex-Time Two-Point Correlation Functions . . . . . . . . . 33

5.2 Ergodic Theorem for some Non-Regular Sequences . . . . . . . . 37

5.3 Diamagnetic Transport Coefficient and Density . . . . . . . . . 41

5.4 Paramagnetic Energy Density . . . . . . . . . . . . . . . . 47

5.5 Hilbert Space of Current Fluctuations . . . . . . . . . . . . . . 63

5.5.1 Positive Sesquilinear Forms _. . . . . . . . . . 63

5.5.2 Hilbert Space and Dynamics . . . . . . . . . . . 66

5.5.3 Averaged Initial State and Dynamics . . . . . . . . . . . 68

\section{Introduction}

Klein, Lenoble and Müller introduced in [KLM] the concept of a "conductivity measure" $\mu_{\mathrm{KLM}}$ for a system of non-interacting fermions subjected to a random potential. They considered the Anderson tight-binding model in presence of a time-dependent spatially homogeneous electric field $\mathcal{E}=\mathcal{E}_{t}$ that is adiabatically switched on. Then they showed that the in-phase linear response current density is, at any time $t \in \mathbb{R}$, given by

$$
J_{\operatorname{lin}}^{\text {in }}(t ; \mathcal{E})=\int_{\mathbb{R}} \hat{\mathcal{E}}_{\nu} \mathrm{e}^{i \nu t} \mu_{\mathrm{KLM}}(\mathrm{d} \nu),
$$

cf. [KLM, Eq. (2.14)]. Here, $\hat{\mathcal{E}}$ is the Fourier transform of $\mathcal{E}$ and is compactly supported. See also [BGKS] for further details on linear response theory of such a model. The fermionic nature of charge carriers - electrons or holes in crystals was implemented by choosing the Fermi-Dirac distribution as the initial ${ }^{1}$ density

\footnotetext{
${ }^{1}$ This corresponds to $t \rightarrow-\infty$ in their approach.
} 
matrix of particles. A conductivity measure can be defined without the localization assumption and at any positive temperature, see $[\mathrm{KM}]$. Inspired by their work, we propose here a notion of (macroscopic) conductivity measure based on the second principle of thermodynamics which corresponds here to the positivity of the heat production for cyclic processes on equilibrium states. In fact, we seek to get a rigorous microscopic description of the phenomenon of linear conductivity from basic principles of thermodynamics (the second one) and quantum mechanics, only.

The present paper belongs to a succession of works on Ohm and Joule's laws starting with [BPK1, BPK2]. Indeed, we mathematically define and analyze in [BPK1] the heat production of the fermion system which is considered here. It is a first preliminary step towards a mathematical description from thermal considerations of transport properties of fermions in disordered media. Then, in [BPK2] we derive Ohm and Joule's laws at the microscopic scale. This second technical step serves as a springboard to the results presented here. Note that in the second paper so-called microscopic conductivity distributions are defined from microscopic conductivity measures. The same construction can be done here to obtain macroscopic conductivity distributions, whose real and imaginary parts satisfy Kramers-Kronig relations. Such arguments are not performed in the present paper because they are already explained in detail in [BPK2, Section 3.5]. The same remark can be done for the derivation of Joule's law in its original formulation under macroscopic electric fields, see [BPK2, Section 4.5]. We present now the mathematical framework we use and our results by only focusing on conductivity measures and current fluctuations. For more details and additional information, see Sections 2-4.

We consider the random two-parameter group $\left\{\mathrm{U}_{t, s}^{(\omega)}\right\}_{t \geq s}$ of unitary operators on $\ell^{2}\left(\mathbb{Z}^{d}\right)$ generated by the time-dependent Hamiltonian

$$
\Delta_{\mathrm{d}}^{(\mathbf{A}(t, \cdot))}+\lambda V_{\omega} \in \mathcal{B}\left(\ell^{2}\left(\mathbb{Z}^{d}\right)\right)
$$

where the parameter $\omega$ runs in a probability space and $\lambda V_{\omega}$ is a random potential with strength $\lambda \in \mathbb{R}_{0}^{+}$(i.e., $\lambda \geq 0$ ). Without electromagnetic potential, i.e., if $\mathbf{A} \equiv 0$, this Hamiltonian corresponds to the Anderson tight-binding model, just as in $[\mathrm{KLM}, \mathrm{KM}]$. The vector potential $\mathbf{A}=\mathbf{A}(t, x) \in C_{0}^{\infty}\left(\mathbb{R} \times \mathbb{R}^{d} ; \mathbb{R}^{d}\right)$ represents a time-dependent spatially inhomogeneous electromagnetic field which is minimally coupled to (minus) the discrete Laplacian $\Delta_{\mathrm{d}}$. We will use in the following the Weyl (or temporal) gauge for the electromagnetic field. In contrast with [KLM, KM], the electromagnetic field is supported in an arbitrarily large but 
bounded region of space and is switched off for times outside some finite interval $\left[t_{0}, t_{1}\right]$.

The family $\left\{\mathrm{U}_{t, s}^{(\omega)}\right\}_{t \geq s}$ of unitary operators on $\ell^{2}\left(\mathbb{Z}^{d}\right)$ induces a random twoparameter group $\left\{\tau_{t, s}^{(\omega)}\right\}_{t \geq s}$ of Bogoliubov automorphisms of a CAR algebra $\mathcal{U}$ associated with (non-relativistic) fermions in the cubic lattice $\mathbb{Z}^{d}$. Indeed, the canonical anti-commutation relations (CAR) encode the Pauli exclusion principle. The $C^{*}$-algebra $\mathcal{U}$ corresponds to a system of (possibly) infinitely many fermions which is infinitely extended. As initial state of the system at time $t_{0} \in \mathbb{R}$, we take the unique KMS state on $\mathcal{U}$ related to the (autonomous) dynamics for $\mathbf{A} \equiv \mathbf{0}$ and inverse temperature $\beta \in \mathbb{R}^{+}$(i.e., $\beta>0$ ). We then analyze this fermion system, which is subjected to a time-dependent electric field, for all times $t \in \mathbb{R}$. However, for the sake of simplicity, in this introduction we present our main results only for times $t \geq t_{1}$ when the electromagnetic field is switched off.

The produced heat up to times $t \geq t_{1}$ is almost surely equal to

$$
\mathbf{Q}(t)=\int_{\mathbb{R}^{d}} \mathrm{~d}^{d} x \int_{t_{0}}^{t} \mathrm{~d} s_{1} \int_{t_{0}}^{s_{1}} \mathrm{~d} s_{2} \boldsymbol{\sigma}\left(s_{1}-s_{2}\right)\left\langle E_{\mathbf{A}}\left(s_{2}, x\right), E_{\mathbf{A}}\left(s_{1}, x\right)\right\rangle \geq 0
$$

at leading order, basically up to terms of order $\mathcal{O}\left(\left|E_{\mathbf{A}}\right|^{3}+\left|\nabla_{x} E_{\mathbf{A}}\right|\left|E_{\mathbf{A}}\right|\right)$. Indeed, $\mathbf{Q}(t)$ is even constant for $t \geq t_{1}$, as, per definition of $t_{1}, E_{\mathbf{A}}(s, x)$ vanishes whenever $s \geq t_{1}$. Here,

$$
E_{\mathbf{A}}(t, x):=-\partial_{t} \mathbf{A}(t, x), \quad t \in \mathbb{R}, x \in \mathbb{R}^{d},
$$

and $\sigma: \mathbb{R} \rightarrow \mathbb{R}$ is a deterministic continuous bounded function that can be made explicit. Compare Equation (2) with [BPK2, Theorem 4.1 (Q), Theorem 5.12 (p)] at times $t \geq t_{1}$. As in [KM], no localization assumption is made. Observe that (minus) the time-derivative of the vector potential $\mathbf{A}$ is the electric field $E_{\mathbf{A}}$ because we use the Weyl gauge. Thus, by interpreting

$$
\int_{t_{0}}^{s_{1}} \boldsymbol{\sigma}\left(s_{1}-s_{2}\right) E_{\mathbf{A}}\left(s_{2}, x\right) \mathrm{d} s_{2}
$$

as the current density at time $s_{1}$ and space position $x \in \mathbb{R}^{d}, \boldsymbol{\sigma}$ can be seen as the conductivity of the system. Hence, (2) is the energy delivered by the electric field to the system in the form predicted by Joule and Ohm's laws.

This interpretation is justified in Section 3 for all $s_{1} \geq t_{0}$. Indeed, by Theorem 3.4, (39) and (71), $\sigma$ is the linear response coefficient associated with the current 
density $J_{\text {lin }}$ induced by a spatially homogeneous time-dependent electric field $\mathcal{E}$ :

$$
J_{\text {lin }}(t)=\int_{t_{0}}^{t} \boldsymbol{\sigma}(t-s) \mathcal{E}_{s} \mathrm{~d} s, \quad t \geq t_{0} .
$$

This equation is nothing but Ohm's law (1) written in time space.

Moreover, in Section 3.3 we show that $\sigma$ is a time correlation function of Bose fields $\Phi_{\mathrm{fl}}\left(\mathbf{i}_{t}\right)$ of current fluctuations $\mathbf{i}_{t}$ at time $t \in \mathbb{R}$ :

$$
\boldsymbol{\sigma}(t)=-4 \operatorname{Im} \varrho_{\mathrm{fl}}\left(\Phi_{\mathrm{fl}}\left(\mathbf{i}_{0}\right) \Phi_{\mathrm{fl}}\left(\int_{0}^{|t|} \mathbf{i}_{s} \mathrm{~d} s\right)\right) .
$$

In particular, (3)-(4) yield Green-Kubo relations. Here, the self-adjoint (unbounded field) operators $\Phi_{\mathrm{fl}}\left(\mathbf{i}_{t}\right)$ generate Weyl operators $\mathrm{e}^{i \Phi_{\mathrm{fl}}\left(\mathbf{i}_{t}\right)}$ of a CCR algebra of normal current fluctuations with respect to (w.r.t.) the initial state. $\varrho_{\mathrm{fl}}$ is an appropriate regular state of this CCR algebra and the right hand side (r.h.s.) of (4) is thus well-defined. This is reminiscent of non-commutative central limit theorems.

It follows from the total heat production (2) that

$$
\int_{t_{0}}^{\infty} \mathrm{d} s_{1} \int_{t_{0}}^{s_{1}} \mathrm{~d} s_{2} \boldsymbol{\sigma}\left(s_{1}-s_{2}\right) \mathcal{E}_{s_{2}} \mathcal{E}_{s_{1}} \geq 0
$$

for any arbitrary smooth compactly supported function $\mathcal{E} \in C_{0}^{\infty}(\mathbb{R} ; \mathbb{R})$ satisfying the so-called AC-condition

$$
\int_{\mathbb{R}} \mathcal{E}_{t} \mathrm{~d} t=0
$$

This condition follows from the fact that $\mathcal{E}$ is the derivative of a smooth function with compact support. Under the form (5)-(6), the positivity of the heat production can be used together with the Bochner-Schwartz theorem [RS2, Theorem IX.10] to obtain the existence of a positive measure $\mu_{\mathrm{AC}}$ of at most polynomial growth such that

$$
\int_{t_{0}}^{\infty} \mathrm{d} s_{1} \int_{t_{0}}^{s_{1}} \mathrm{~d} s_{2} \boldsymbol{\sigma}\left(s_{1}-s_{2}\right) \mathcal{E}_{s_{2}} \mathcal{E}_{s_{1}}=\int_{\mathbb{R} \backslash\{0\}}\left|\hat{\mathcal{E}}_{\nu}\right|^{2} \mu_{\mathrm{AC}}(\mathrm{d} \nu)
$$

for all $\mathcal{E} \in C_{0}^{\infty}(\mathbb{R} ; \mathbb{R})$ obeying (6), with $\hat{\mathcal{E}}$ being the Fourier transform of $\mathcal{E}$.

The measure $\mu_{\mathrm{AC}}$ is naturally named in-phase AC-conductivity measure of the fermion system as $\left|\hat{\mathcal{E}}_{\nu}\right|^{2} \mu_{\mathrm{AC}}(\mathrm{d} \nu)$ is the heat production due to the component 
of frequency $\nu$ of the electric field, in accordance with Joule's law in the ACregime. Its properties will be studied in a subsequent paper. For instance, we will show that $\mu_{\mathrm{AC}}(\mathbb{R} \backslash\{0\})>0$, at least for large temperatures $T=\beta^{-1}$ and small randomness $\lambda>0$.

To conclude, our main assertions are Theorems 3.1 (charge transport coefficients), 3.4 (Ohm's law), 3.6 (Green-Kubo relations and current fluctuations), 4.1 (Joule's law) and 4.3 (AC-conductivity measure). This paper is organized as follows:

- In Section 2 we define our model and highlight the relations between our approach and [KLM, KM].

- We define in Section 3 a CCR algebra of fluctuations intimately related to Ohm's law.

- Section 4 states Joule's law from which we deduce the existence of the (macroscopic) AC-conductivity measure.

- Section 5 gathers technical proofs on which Sections 3-4 are based. The arguments strongly use the results of [BPK1, BPK2].

\section{Notation 1.1 (Generic constants)}

To simplify notation, we denote by $D$ any generic positive and finite constant. These constants do not need to be the same from one statement to another.

\section{Setup of the Problem}

Up to the probability space, the mathematical setting of our study, including notation, is taken from [BPK1, BPK2]. For the reader's convenience and completeness, we now briefly recall it and highlight the relations to the mathematical framework of [KLM]. For further details we refer to [BPK1, BPK2].

\subsection{Anderson Tight-Binding Model}

The $d$-dimensional cubic lattice $\mathfrak{L}:=\mathbb{Z}^{d}(d \in \mathbb{N})$ represents the crystal and we define $\mathcal{P}_{f}(\mathfrak{L}) \subset 2^{\mathfrak{L}}$ to be the set of all finite subsets of $\mathfrak{L}$.

Disorder in the crystal is modeled by a random potential coming from a probability space $\left(\Omega, \mathfrak{A}_{\Omega}, \mathfrak{a}_{\Omega}\right)$ defined as follows: Let $\Omega:=[-1,1]^{\mathfrak{L}}$ and $\Omega_{x}, x \in \mathfrak{L}$, 
be an arbitrary element of the Borel $\sigma$-algebra of the interval $[-1,1]$ w.r.t. the usual metric topology. Then, $\mathfrak{A}_{\Omega}$ is the $\sigma$-algebra generated by the cylinder sets $\prod_{x \in \mathfrak{L}} \Omega_{x}$, where $\Omega_{x}=[-1,1]$ for all but finitely many $x \in \mathfrak{L}$. The measure $\mathfrak{a}_{\Omega}$ is the product measure

$$
\mathfrak{a}_{\Omega}\left(\prod_{x \in \mathfrak{L}} \Omega_{x}\right):=\prod_{x \in \mathfrak{L}} \mathfrak{a}_{\mathbf{0}}\left(\Omega_{x}\right),
$$

where $\mathfrak{a}_{\mathbf{0}}$ is any fixed probability measure on the interval $[-1,1]$. In other words, the random variables are independently and identically distributed (i.i.d.). We denote by $\mathbb{E}[\cdot]$ the expectation value associated with $\mathfrak{a}_{\Omega}$. Note that the i.i.d. property of the random variables is not essential for our results. We could take any ergodic ensemble instead. In fact, the i.i.d. property is mainly used to simplify the arguments of Section 4.3.

For any realization $\omega \in \Omega, V_{\omega} \in \mathcal{B}\left(\ell^{2}(\mathfrak{L})\right)$ is the self-adjoint multiplication operator with the function $\omega: \mathfrak{L} \rightarrow[-1,1]$. Then, we consider the Anderson tight-binding Hamiltonian $\left(\Delta_{\mathrm{d}}+\lambda V_{\omega}\right)$ acting on the Hilbert space $\ell^{2}(\mathfrak{L})$, where $\Delta_{\mathrm{d}} \in \mathcal{B}\left(\ell^{2}(\mathfrak{L})\right)$ is (up to a minus sign) the usual $d$-dimensional discrete Laplacian defined by

$$
\left[\Delta_{\mathrm{d}}(\psi)\right](x):=2 d \psi(x)-\sum_{z \in \mathfrak{L},|z|=1} \psi(x+z), \quad x \in \mathfrak{L}, \psi \in \ell^{2}(\mathfrak{L}) .
$$

Note that we could add some constant (chemical) potential to the discrete Laplacian without changing our proofs. We will use in particular the random unitary group $\left\{\mathrm{U}_{t}^{(\omega, \lambda)}\right\}_{t \in \mathbb{R}}$ generated by the Hamiltonians $\left(\Delta_{\mathrm{d}}+\lambda V_{\omega}\right)$ for $\omega \in \Omega$ and $\lambda \in \mathbb{R}_{0}^{+}$:

$$
\mathrm{U}_{t}^{(\omega, \lambda)}:=\exp \left(-i t\left(\Delta_{\mathrm{d}}+\lambda V_{\omega}\right)\right) \in \mathcal{B}\left(\ell^{2}(\mathfrak{L})\right), \quad t \in \mathbb{R} .
$$

This group defines our free one-particle dynamics, like in [KLM].

\subsection{Coupling to Electromagnetic Fields}

The electromagnetic potential is defined by a compactly supported potential

$$
\mathbf{A} \in \mathbf{C}_{0}^{\infty}=\bigcup_{l \in \mathbb{R}^{+}} C_{0}^{\infty}\left(\mathbb{R} \times[-l, l]^{d} ;\left(\mathbb{R}^{d}\right)^{*}\right) .
$$

Here, $\left(\mathbb{R}^{d}\right)^{*}$ is the set of one-forms ${ }^{2}$ on $\mathbb{R}^{d}$ that take values in $\mathbb{R}$ and $\mathbf{A}(t, x) \equiv 0$ whenever $x \notin[-l, l]^{d}$ and $\mathbf{A} \in C_{0}^{\infty}\left(\mathbb{R} \times[-l, l]^{d} ;\left(\mathbb{R}^{d}\right)^{*}\right)$. Using any orthonormal

\footnotetext{
${ }^{2}$ In a strict sense, one should take the dual space of the tangent spaces $T\left(\mathbb{R}^{d}\right)_{x}, x \in \mathbb{R}^{d}$.
} 
basis $\left\{e_{k}\right\}_{k=1}^{d}$ of the Euclidian space $\mathbb{R}^{d}$, we define the scalar product between two fields $E^{(1,2)} \in\left(\mathbb{R}^{d}\right)^{*}$ as usual by

$$
\left\langle E^{(1)}, E^{(2)}\right\rangle:=\sum_{k=1}^{d} E^{(1)}\left(e_{k}\right) E^{(2)}\left(e_{k}\right) .
$$

Since $\mathbf{A} \in \mathbf{C}_{0}^{\infty}, \mathbf{A}(t, x)=0$ for all $t \leq t_{0}$, where $t_{0} \in \mathbb{R}$ is some initial time. We use the Weyl gauge for the electromagnetic field and as a consequence,

$$
E_{\mathbf{A}}(t, x):=-\partial_{t} \mathbf{A}(t, x), \quad t \in \mathbb{R}, x \in \mathbb{R}^{d},
$$

is the electric field associated with $\mathbf{A}$.

Remark 2.1 To simplify notation, we identify in the sequel $\left(\mathbb{R}^{d}\right)^{*}$ with $\mathbb{R}^{d}$ via the canonical scalar product of $\mathbb{R}^{d}$.

Since $\mathbf{A}$ is by assumption compactly supported, the corresponding electric field satisfies the $A C$-condition

$$
\int_{t_{0}}^{t} E_{\mathbf{A}}(s, x) \mathrm{d} s=0, \quad x \in \mathbb{R}^{d}
$$

for sufficiently large times $t \geq t_{1} \geq t_{0}$. From (13)

$$
t_{1}:=\min \left\{t \geq t_{0}: \quad \int_{t_{0}}^{t^{\prime}} E_{\mathbf{A}}(s, x) \mathrm{d} s=0 \quad \text { for all } x \in \mathbb{R}^{d} \text { and } t^{\prime} \geq t\right\}
$$

is the (arbitrary) time at which the electric field is turned off.

We consider without loss of generality (w.l.o.g.) negatively charged fermions. Thus, using the (minimal) coupling of $\mathbf{A} \in \mathbf{C}_{0}^{\infty}$ to the discrete Laplacian $-\Delta_{\mathrm{d}}$, the discrete time-dependent magnetic Laplacian is (up to a minus sign) the selfadjoint operator

$$
\Delta_{\mathrm{d}}^{(\mathbf{A})} \equiv \Delta_{\mathrm{d}}^{(\mathbf{A}(t, \cdot))} \in \mathcal{B}\left(\ell^{2}(\mathfrak{L})\right), \quad t \in \mathbb{R}
$$

defined by

$$
\left\langle\mathfrak{e}_{x}, \Delta_{\mathrm{d}}^{(\mathbf{A})} \mathfrak{e}_{y}\right\rangle=\exp \left(-i \int_{0}^{1}[\mathbf{A}(t, \alpha y+(1-\alpha) x)](y-x) \mathrm{d} \alpha\right)\left\langle\mathfrak{e}_{x}, \Delta_{\mathrm{d}} \mathfrak{e}_{y}\right\rangle
$$


for all $t \in \mathbb{R}$ and $x, y \in \mathfrak{L}$. Here, $\langle\cdot, \cdot\rangle$ is the scalar product in $\ell^{2}(\mathfrak{L})$ and $\left\{\mathfrak{e}_{x}\right\}_{x \in \mathfrak{L}}$ is the canonical orthonormal basis $\mathfrak{e}_{x}(y) \equiv \delta_{x, y}$ of $\ell^{2}(\mathfrak{L})$. In (15), $\alpha y+(1-\alpha) x$ and $y-x$ are seen as elements of $\mathbb{R}^{d}$.

Therefore, in presence of electromagnetic fields, the Anderson tight-binding Hamiltonian $\left(\Delta_{\mathrm{d}}+\lambda V_{\omega}\right)$ for $\omega \in \Omega$ and $\lambda \in \mathbb{R}_{0}^{+}$is replaced by the time-dependent one $\left(\Delta_{\mathrm{d}}^{(\mathbf{A})}+\lambda V_{\omega}\right)$. As explained in [BPK1, Section 2.3], the interaction between magnetic fields and electron spins is here neglected because such a term becomes negligible for electromagnetic potentials slowly varying in space. This yields a perturbed dynamics defined by the random two-parameter group $\left\{\mathrm{U}_{t, s}^{(\omega, \lambda, \mathbf{A})}\right\}_{t \geq s}$ of unitary operators on $\ell^{2}(\mathfrak{L})$ which is the unique solution, for any $\omega \in \Omega, \lambda \in \mathbb{R}_{0}^{+}$ and $\mathbf{A} \in \mathbf{C}_{0}^{\infty}$, of the non-autonomous evolution equation

$\forall s, t \in \mathbb{R}, t \geq s: \quad \partial_{t} \mathrm{U}_{t, s}^{(\omega, \lambda, \mathbf{A})}=-i\left(\Delta_{\mathrm{d}}^{(\mathbf{A}(t, \cdot))}+\lambda V_{\omega}\right) \mathrm{U}_{t, s}^{(\omega, \lambda, \mathbf{A})}, \quad \mathrm{U}_{s, s}^{(\omega, \lambda, \mathbf{A})}:=\mathbf{1}$.

The physical situation considered here to investigate Ohm and Joule's laws is as follows: We start with a macroscopic bulk containing conducting fermions. This is idealized by taking the one-particle Hilbert space $\ell^{2}(\mathfrak{L})$. Then, the heat production or the conductivity is measured in a local region which is very small w.r.t. the size of the bulk, but very large w.r.t. the lattice spacing of the crystal. We implement this hierarchy of space scales by rescaling the vector potentials. That means, for any $l \in \mathbb{R}^{+}$and $\mathbf{A} \in \mathbf{C}_{0}^{\infty}$, we consider the space-rescaled vector potential

$$
\mathbf{A}_{l}(t, x):=\mathbf{A}\left(t, l^{-1} x\right), \quad t \in \mathbb{R}, x \in \mathbb{R}^{d} .
$$

Then, to ensure that a macroscopic number of lattice sites is involved, we eventually perform the limit $l \rightarrow \infty$. Indeed, the scaling factor $l^{-1}$ used in (17) means, at fixed $l$, that the space scale of the electric field (12) is infinitesimal w.r.t. the macroscopic bulk (which is the whole space), whereas the lattice spacing gets infinitesimal w.r.t. the space scale of the vector potential when $l \rightarrow \infty$. Furthermore, Ohm's law is a linear response to electric fields. Therefore, we also rescale the strength of the electromagnetic potential $\mathbf{A}_{l}$ by a parameter $\eta \in \mathbb{R}$ and eventually take the limit $\eta \rightarrow 0$. All together, this yields the random two-parameter group $\left\{\mathrm{U}_{t, s}^{\left(\omega, \lambda, \eta \mathbf{A}_{l}\right)}\right\}_{t \geq s}$ to be studied in the limit $l \rightarrow \infty, \eta \rightarrow 0$. For more discussions, see [BPK2, Section 2.3].

Recall that in [KLM, KM] the authors use a time-dependent spatially homogeneous electric field that is adiabatically switched on. This situation is thus rather different from our study where the electromagnetic field is supported in an arbitrarily large but bounded region of space and is switched off for times outside the finite interval $\left[t_{0}, t_{1}\right]$. 


\subsection{Algebraic Approach}

Although there is no interaction between fermions, we do not restrict our analyses to the one-particle Hilbert space to study transport properties. Instead, our approach is based on the algebraic formulation of fermion systems on lattices because it makes the role played by many-fermion correlations due to the Pauli exclusion principle, i.e., the antisymmetry of the many-body wave function, more transparent:

- The positivity required for the existence of the in-phase AC-conductivity measure is directly related to the passivity property of (thermal equilibrium) states on the CAR algebra $\mathcal{U}$ defined below.

- The conductivity is naturally defined from current-current correlations, that is, four-point correlation functions, in this framework.

- The algebraic formulation allows a clear link between transport properties of fermion systems and the CCR algebra of current fluctuations. The latter is related to non-commutative central limit theorems (see, e.g., [GVV]).

- Moreover, this approach can be naturally used to define conductivity measures for interacting fermions as well. This paper can thus be seen as a preparation for such mathematical studies.

The CAR $C^{*}$-algebra of the infinite system is denoted by $\mathcal{U}$. We define annihilation and creation operators of (spinless) fermions with wave functions $\psi \in \ell^{2}(\mathfrak{L})$ by

$$
a(\psi):=\sum_{x \in \mathfrak{L}} \overline{\psi(x)} a_{x} \in \mathcal{U}, \quad a^{*}(\psi):=\sum_{x \in \mathfrak{L}} \psi(x) a_{x}^{*} \in \mathcal{U} .
$$

Here, $a_{x}, a_{x}^{*}, x \in \mathfrak{L}$, and the identity 1 are generators of $\mathcal{U}$ and satisfy the canonical anti-commutation relations.

For all $\omega \in \Omega$ and $\lambda \in \mathbb{R}_{0}^{+}$, the free dynamics on $\mathcal{U}$ is defined by the unique one-parameter strongly continuous group $\tau^{(\omega, \lambda)}:=\left\{\tau_{t}^{(\omega, \lambda)}\right\}_{t \in \mathbb{R}}$ of (Bogoliubov) automorphisms of $\mathcal{U}$ satisfying the condition

$$
\tau_{t}^{(\omega, \lambda)}(a(\psi))=a\left(\left(\mathrm{U}_{t}^{(\omega, \lambda)}\right)^{*}(\psi)\right), \quad t \in \mathbb{R}, \psi \in \ell^{2}(\mathfrak{L}) .
$$

See (10) and [BR2, Theorem 5.2.5]. 
Similarly, in presence of electromagnetic potentials $\mathbf{A} \in \mathbf{C}_{0}^{\infty}$ the dynamics on $\mathcal{U}$ is defined by the unique family $\left\{\tau_{t, s}^{(\omega, \lambda, \mathbf{A})}\right\}_{t \geq s}$ of random (Bogoliubov) automorphisms with

$$
\tau_{t, s}^{(\omega, \lambda, \mathbf{A})}(a(\psi))=a\left(\left(\mathrm{U}_{t, s}^{(\omega, \lambda, \mathbf{A})}\right)^{*}(\psi)\right), \quad t \geq s, \psi \in \ell^{2}(\mathfrak{L}),
$$

for all $\omega \in \Omega, \lambda \in \mathbb{R}_{0}^{+}$. See (16) and [BR2, Theorem 5.2.5]. The family $\left\{\tau_{t, s}^{(\omega, \lambda, \mathbf{A})}\right\}_{t \geq s}$ is itself the solution of a non-autonomous evolution equation, see [BPK1, Sections 5.2-5.3].

States on the $C^{*}$-algebra $\mathcal{U}$ are, by definition, continuous linear functionals $\rho \in \mathcal{U}^{*}$ which are normalized and positive, i.e., $\rho(\mathbf{1})=1$ and $\rho\left(A^{*} A\right) \geq 0$ for all $A \in \mathcal{U}$. As explained for instance in [BPK1, Section 2.5], the thermodynamic equilibrium of the system at inverse temperature $\beta \in \mathbb{R}^{+}$(i.e., $\beta>0$ ) is described by the unique $\left(\tau^{(\omega, \lambda)}, \beta\right)$-KMS state $\varrho^{(\beta, \omega, \lambda)}$. See also [BR2, Example 5.3.2.] or [AJP, Theorem 5.9]. The choice of KMS states as thermal equilibrium states is sustained by the second principle of thermodynamics [PW]. See discussions of Section 4. It is well-known that such states are stationary w.r.t. the dynamics, that is,

$$
\varrho^{(\beta, \omega, \lambda)} \circ \tau_{t}^{(\omega, \lambda)}=\varrho^{(\beta, \omega, \lambda)}, \quad \beta \in \mathbb{R}^{+}, \omega \in \Omega, \lambda \in \mathbb{R}_{0}^{+}, t \in \mathbb{R} .
$$

Since $\mathbf{A}(t, x)=0$ for all $t \leq t_{0}$, the time evolution of the state of the system is thus

$$
\rho_{t}^{(\beta, \omega, \lambda, \mathbf{A})}:=\left\{\begin{array}{lll}
\varrho^{(\beta, \omega, \lambda)} & , & t \leq t_{0}, \\
\varrho^{(\beta, \omega, \lambda)} \circ \tau_{t, t_{0}}^{(\omega, \lambda, \mathbf{A})}, & t \geq t_{0} .
\end{array}\right.
$$

This time-evolving state is quasi-free by construction for all times. Such quasifree states are uniquely characterized by bounded positive operators $\mathbf{d} \in \mathcal{B}\left(\ell^{2}(\mathfrak{L})\right)$ obeying $0 \leq \mathbf{d} \leq \mathbf{1}$. These operators are named symbols of the corresponding states. The symbol of $\varrho^{(\beta, \omega, \lambda)}$ is

$$
\mathbf{d}_{\mathrm{fermi}}^{(\beta, \omega, \lambda)}:=\frac{1}{1+\mathrm{e}^{\beta\left(\Delta_{\mathrm{d}}+\lambda V_{\omega}\right)}} \in \mathcal{B}\left(\ell^{2}(\mathfrak{L})\right) .
$$

We infer from the definitions (16), (20) and (22) together with the evolution law (16) that the symbol $\mathbf{d}_{t}^{(\beta, \omega, \lambda, \mathbf{A})}$ of the quasi-free state $\rho_{t}^{(\beta, \omega, \lambda, \mathbf{A})}$ is the solution to the Liouville equation

$$
\forall t \geq t_{0}: \quad \partial_{t} \mathbf{d}_{t}^{(\beta, \omega, \lambda, \mathbf{A})}=-i\left[\Delta_{\mathrm{d}}^{(\mathbf{A})}+\lambda V_{\omega}, \mathbf{d}_{t}^{(\beta, \omega, \lambda, \mathbf{A})}\right], \quad \mathbf{d}_{t_{0}}^{(\beta, \omega, \lambda, \mathbf{A})}:=\mathbf{d}_{\mathrm{fermi}}^{(\beta, \omega, \lambda)}
$$


for every realization $\omega \in \Omega, \lambda \in \mathbb{R}_{0}^{+}$and $\beta \in \mathbb{R}^{+}$. In [BGKS, KLM, KM] the authors consider an evolution equation similar to (24) with $t_{0}=-\infty$ and use the expectation value of the velocity observable w.r.t. the trace per unit volume of $\mathbf{d}_{t}^{(\beta, \omega, \lambda, \mathbf{A})} \in \mathcal{B}\left(\ell^{2}(\mathfrak{L})\right)$ to define a current density. See, e.g., [KLM, Eqs. (2.5)(2.6)]. Spatially local perturbations $\mathbf{A} \in \mathbf{C}_{0}^{\infty}$ of the electromagnetic field do not influence the mean velocity of an infinite system of particles. Thus, by contrast, the electromagnetic perturbation considered in [BGKS, KLM, KM] is infinitely extended as it is space-homogeneous. Indeed, w.r.t. the time-evolving density operator $\mathbf{d}_{t}^{(\beta, \omega, \lambda, \mathbf{A})}$, the main quantities we analyze are not trace densities, but rather the infinite volume limit of (finite volume) traces, see, e.g., (56) below. Note however that, by considering space-homogeneous electromagnetic perturbations $\mathrm{A}_{l}$ in finite boxes $\Lambda_{l}$ and the corresponding current densities, up to the different convention on $\mathbf{d}_{t}^{(\beta, \omega, \lambda, \mathbf{A})}$ for the initial condition, one would obtain in the limit $l \rightarrow \infty$ a notion of conductivity corresponding quite well to the one introduced in [KLM, Eqs. (2.5)-(2.6)], even if this correspondence is not totally explicit and the approaches are conceptually different. See also discussions around (38).

\section{CCR Algebra of Fluctuations of Ohm's Law}

The study of classical (macroscopic) Ohm's law for fermions within disordered media leads us to consider a CCR $C^{*}$-algebra of current fluctuations. Exactly like in [BPK2, Section 3], we only consider space-homogeneous (though timedependent) electric fields in the box

$$
\Lambda_{l}:=\left\{\left(x_{1}, \ldots, x_{d}\right) \in \mathfrak{L}:\left|x_{1}\right|, \ldots,\left|x_{d}\right| \leq l\right\} \in \mathcal{P}_{f}(\mathfrak{L})
$$

with $l \in \mathbb{R}^{+}$. More precisely, let $\vec{w}:=\left(w_{1}, \ldots, w_{d}\right) \in \mathbb{R}^{d}$ be any (normalized) vector, $\mathcal{A} \in C_{0}^{\infty}(\mathbb{R} ; \mathbb{R})$ and set $\mathcal{E}_{t}:=-\partial_{t} \mathcal{A}_{t}$ for all $t \in \mathbb{R}$. Then, $\overline{\mathbf{A}} \in \mathbf{C}_{0}^{\infty}$ is defined to be the electromagnetic potential whose electric field equals $\mathcal{E}_{t} \vec{w}$ at time $t \in \mathbb{R}$ for all $x \in[-1,1]^{d}$, and $(0,0, \ldots, 0)$ for $t \in \mathbb{R}$ and $x \notin[-1,1]^{d}$. See (72)-(74) for more details. This choice yields rescaled electromagnetic potentials $\eta \overline{\mathbf{A}}_{l}$ as defined by (17) for $l \in \mathbb{R}^{+}$and $\eta \in \mathbb{R}$.

\subsection{Macroscopic Transport Coefficients}

For any pair $\mathbf{x}:=\left(x^{(1)}, x^{(2)}\right) \in \mathfrak{L}^{2}$, we define the paramagnetic and diamagnetic current observables $I_{\mathbf{x}}=I_{\mathbf{x}}^{*}$ and $\mathrm{I}_{\mathbf{x}}^{\mathbf{A}}=\left(\mathrm{I}_{\mathbf{x}}^{\mathbf{A}}\right)^{*}$ for $\mathbf{A} \in \mathbf{C}_{0}^{\infty}$ at time $t \in \mathbb{R}$ by

$$
I_{\mathbf{x}}:=-2 \operatorname{Im}\left(a_{x^{(2)}}^{*} a_{x^{(1)}}\right)=i\left(a_{x^{(2)}}^{*} a_{x^{(1)}}-a_{x^{(1)}}^{*} a_{x^{(2)}}\right)
$$


and

$$
\mathrm{I}_{\mathbf{x}}^{\mathbf{A}}:=-2 \operatorname{Im}\left(\left(\mathrm{e}^{-i \int_{0}^{1}\left[\mathbf{A}\left(t, \alpha x^{(2)}+(1-\alpha) x^{(1)}\right)\right]\left(x^{(2)}-x^{(1)}\right) \mathrm{d} \alpha}-1\right) a_{x^{(2)}}^{*} a_{x^{(1)}}\right) .
$$

Here, $I_{(x, y)}$ is the observable related to the flow of negatively charged particles from the lattice site $x$ to the lattice site $y$ or the current from $y$ to $x$ without external electromagnetic potential. $\mathrm{I}_{\mathrm{x}}^{\mathbf{A}}$ is the current observable corresponding to the acceleration of charged particles induced by the electromagnetic field. See [BPK2, Section 3.1] for more details. We also denote by

$$
P_{\mathbf{x}}:=a_{x^{(2)}}^{*} a_{x^{(1)}}+a_{x^{(1)}}^{*} a_{x^{(2)}}, \quad \mathbf{x}:=\left(x^{(1)}, x^{(2)}\right) \in \mathfrak{L}^{2},
$$

the second-quantization of the adjacency matrix of the oriented graph containing exactly the edges $\left(x^{(2)}, x^{(1)}\right)$ and $\left(x^{(1)}, x^{(2)}\right)$.

Now, for any $\beta \in \mathbb{R}^{+}, \omega \in \Omega$ and $\lambda \in \mathbb{R}_{0}^{+}$we define two important functions associated with the observables $I_{\mathrm{x}}$ and $P_{\mathrm{x}}$ :

(p) The paramagnetic transport coefficient $\sigma_{\mathrm{p}}^{(\omega)} \equiv \sigma_{\mathrm{p}}^{(\beta, \omega, \lambda)}$ is defined by

$$
\sigma_{\mathrm{p}}^{(\omega)}(\mathbf{x}, \mathbf{y}, t):=\int_{0}^{t} \varrho^{(\beta, \omega, \lambda)}\left(i\left[I_{\mathbf{y}}, \tau_{s}^{(\omega, \lambda)}\left(I_{\mathbf{x}}\right)\right]\right) \mathrm{d} s, \quad \mathbf{x}, \mathbf{y} \in \mathfrak{L}^{2}, t \in \mathbb{R} .
$$

(d) The diamagnetic transport coefficient $\sigma_{\mathrm{d}}^{(\omega)} \equiv \sigma_{\mathrm{d}}^{(\beta, \omega, \lambda)}$ is defined by

$$
\sigma_{\mathrm{d}}^{(\omega)}(\mathbf{x}):=\varrho^{(\beta, \omega, \lambda)}\left(P_{\mathbf{x}}\right), \quad \mathbf{x} \in \mathfrak{L}^{2} .
$$

As explained in [BPK2, Section 3.3], $\sigma_{\mathrm{p}}^{(\omega)}$ is related with a quantum current viscosity whereas $\sigma_{\mathrm{d}}^{(\omega)}$ describes the ballistic movement of charged particles within the electric field.

For large regions $\Lambda_{l} \subset \mathfrak{L}$, we then define the space-averaged paramagnetic transport coefficient

$$
t \mapsto \Xi_{\mathrm{p}, l}^{(\omega)}(t) \equiv \Xi_{\mathrm{p}, l}^{(\beta, \omega, \lambda)}(t) \in \mathcal{B}\left(\mathbb{R}^{d}\right)
$$

w.r.t. the canonical orthonormal basis $\left\{e_{k}\right\}_{k=1}^{d}$ of the Euclidian space $\mathbb{R}^{d}$ by

$$
\left\{\Xi_{\mathrm{p}, l}^{(\omega)}(t)\right\}_{k, q}:=\frac{1}{\left|\Lambda_{l}\right|} \sum_{x, y \in \Lambda_{l}} \sigma_{\mathrm{p}}^{(\omega)}\left(x+e_{q}, x, y+e_{k}, y, t\right)
$$


for any $l, \beta \in \mathbb{R}^{+}, \omega \in \Omega, \lambda \in \mathbb{R}_{0}^{+}, k, q \in\{1, \ldots, d\}$ and $t \in \mathbb{R}$. Similarly, the space-averaged diamagnetic transport coefficient

$$
\Xi_{\mathrm{d}, l}^{(\omega)} \equiv \Xi_{\mathrm{d}, l}^{(\beta, \omega, \lambda)} \in \mathcal{B}\left(\mathbb{R}^{d}\right)
$$

corresponds, w.r.t. the canonical orthonormal basis $\left\{e_{k}\right\}_{k=1}^{d}$, to the diagonal matrix

$$
\left\{\Xi_{\mathrm{d}, l}^{(\omega)}\right\}_{k, q}:=\frac{\delta_{k, q}}{\left|\Lambda_{l}\right|} \sum_{x \in \Lambda_{l}} \sigma_{\mathrm{d}}^{(\omega)}\left(x+e_{k}, x\right) \in[-2,2] .
$$

See [BPK2, Eq. (37), Theorem 3.1, Corollary 3.2] for details on the mathematical properties of these random transport coefficients. They are directly linked to Ohm's law as explained in [BPK2, Theorem 3.3] and it is natural to consider their expectation values:

We define the deterministic paramagnetic transport coefficient

$$
t \mapsto \boldsymbol{\Xi}_{\mathrm{p}}(t) \equiv \boldsymbol{\Xi}_{\mathrm{p}}^{(\beta, \lambda)}(t) \in \mathcal{B}\left(\mathbb{R}^{d}\right)
$$

by

$$
\Xi_{\mathrm{p}}(t):=\lim _{l \rightarrow \infty} \mathbb{E}\left[\Xi_{\mathrm{p}, l}^{(\omega)}(t)\right]
$$

for any $\beta \in \mathbb{R}^{+}, \lambda \in \mathbb{R}_{0}^{+}, k, q \in\{1, \ldots, d\}$ and $t \in \mathbb{R}$. This transport coefficient is well-defined, see, e.g., Equation (146). Furthermore, the convergence is uniform w.r.t. times $t$ in compact sets. By [BPK2, Corollary 3.2 (i)-(ii) and (iv)], $\boldsymbol{\Xi}_{\mathrm{p}} \in$ $C\left(\mathbb{R} ; \mathcal{B}_{-}\left(\mathbb{R}^{d}\right)\right)$ and $\boldsymbol{\Xi}_{\mathrm{p}}(t)=\boldsymbol{\Xi}_{\mathrm{p}}(|t|)$ with $\boldsymbol{\Xi}_{\mathrm{p}}(0)=0$. Here, $\mathcal{B}_{-}\left(\mathbb{R}^{d}\right)$ is the set of negative linear operators on $\mathbb{R}^{d}$. Analogously, we also introduce the deterministic diamagnetic transport coefficient

$$
\boldsymbol{\Xi}_{\mathrm{d}} \equiv \boldsymbol{\Xi}_{\mathrm{d}}^{(\beta, \lambda)} \in \mathcal{B}\left(\mathbb{R}^{d}\right)
$$

defined, for any $\beta \in \mathbb{R}^{+}$and $\lambda \in \mathbb{R}_{0}^{+}$, by

$$
\Xi_{\mathrm{d}}:=\lim _{l \rightarrow \infty} \mathbb{E}\left[\Xi_{\mathrm{d}, l}^{(\omega)}\right]
$$

Indeed, by translation invariance of the probability measure $\mathfrak{a}_{\Omega}$ and the uniqueness of the KMS states $\rho^{(\beta, \omega, \lambda)}$, we even have, for all $l>0$,

$$
\Xi_{\mathrm{d}}=\mathbb{E}\left[\Xi_{\mathrm{d}, l}^{(\omega)}\right]
$$

By using the Akcoglu-Krengel ergodic theorem (cf. Theorem 5.4) we show that the limits $l \rightarrow \infty$ of $\Xi_{\mathrm{p}, l}^{(\omega)}$ and $\Xi_{\mathrm{d}, l}^{(\omega)}$ converge almost surely to $\boldsymbol{\Xi}_{\mathrm{p}}$ and $\boldsymbol{\Xi}_{\mathrm{d}}$ : 


\section{Theorem 3.1 (Macroscopic charge transport coefficients)}

Let $\beta \in \mathbb{R}^{+}$and $\lambda \in \mathbb{R}_{0}^{+}$. Then, there is a measurable subset $\tilde{\Omega} \equiv \tilde{\Omega}^{(\beta, \lambda)} \subset \Omega$ of full measure such that, for any $\omega \in \tilde{\Omega}$, one has:

(p) Paramagnetic charge transport coefficient: For all $t \in \mathbb{R}$,

$$
\boldsymbol{\Xi}_{\mathrm{p}}(t)=\lim _{l \rightarrow \infty} \Xi_{\mathrm{p}, l}^{(\omega)}(t) \in \mathcal{B}_{-}\left(\mathbb{R}^{d}\right) .
$$

The limit above is uniform for times $t$ on compact sets.

(d) Diamagnetic charge transport coefficient:

$$
\boldsymbol{\Xi}_{\mathrm{d}}=\lim _{l \rightarrow \infty} \Xi_{\mathrm{d}, l}^{(\omega)} \in \mathcal{B}\left(\mathbb{R}^{d}\right), \quad\left\{\boldsymbol{\Xi}_{\mathrm{d}}\right\}_{k, k} \in[-2,2], k \in\{1, \ldots, d\}
$$

Proof: (p) Take electric fields which equal $\vec{w}:=\left(w_{1}, \ldots, w_{d}\right) \in \mathbb{R}^{d}$ at time $t \in \mathbb{R}$ for all $x \in[-1,1]^{d}$ and $(0,0, \ldots, 0)$ for $t \in \mathbb{R}$ and $x \notin[-1,1]^{d}$. Then, the first assertion is a direct consequence of (31), (33), (121), Theorem 5.19 and Lemma 5.22 combined with [BPK2, Lemma 5.2].

(d) is Corollary 5.7 (ii). Note additionally that the intersection of two measurable sets of full measure has full measure.

In [BPK2, Eq. (47)] we introduce the (linear) conductivity $\Sigma_{l}^{(\omega)}$ of the fermion system in the box $\Lambda_{l}$ from its paramagnetic and diamagnetic charge transport coefficients. Exactly in the same way, we define the macroscopic conductivity $\Sigma$ as follows:

\section{Definition 3.2 (Macroscopic conductivity)}

For $\beta \in \mathbb{R}^{+}$and $\lambda \in \mathbb{R}_{0}^{+}$, the macroscopic conductivity is the map

$$
t \mapsto \boldsymbol{\Sigma}(t) \equiv \boldsymbol{\Sigma}^{(\beta, \lambda)}(t):= \begin{cases}0 & t \leq 0 \\ \boldsymbol{\Xi}_{\mathrm{d}}+\boldsymbol{\Xi}_{\mathrm{p}}(t), & t \geq 0\end{cases}
$$

Indeed, by Theorem 3.1, the local conductivity $\Sigma_{l}^{(\omega)}$ defined by [BPK2, Eq. (47)] converges almost surely to the macroscopic conductivity $\Sigma$, as $l \rightarrow \infty$.

\section{Remark 3.3 (Current viscosity)}

For $\beta \in \mathbb{R}^{+}, \lambda \in \mathbb{R}_{0}^{+}$and $t \in \mathbb{R}$, the quantity

$$
\mathbf{V}(t):=\left(\boldsymbol{\Xi}_{\mathrm{d}}\right)^{-1} \partial_{t} \boldsymbol{\Xi}_{\mathrm{p}}(t) \in \mathcal{B}\left(\mathbb{R}^{d}\right)
$$

defines a macroscopic current viscosity, similar to [BPK2, Eq. (40)]. 


\subsection{Classical Ohm's Law}

For any $l, \beta \in \mathbb{R}^{+}, \omega \in \Omega, \lambda \in \mathbb{R}_{0}^{+}, \eta \in \mathbb{R}, \vec{w} \in \mathbb{R}^{d}$ and $\mathcal{A} \in C_{0}^{\infty}(\mathbb{R} ; \mathbb{R})$, the current density due to the space-homogeneous electric perturbation $\mathcal{E}$ in the box $\Lambda_{l}$ is the sum of three current densities defined from (26)-(27):

(th) The thermal current density

$$
\mathbb{J}_{\mathrm{th}}^{(\omega, l)} \equiv \mathbb{J}_{\mathrm{th}}^{(\beta, \omega, \lambda, l)} \in \mathbb{R}^{d}
$$

at equilibrium inside the box $\Lambda_{l}$ is defined, for any $k \in\{1, \ldots, d\}$, by

$$
\left\{\mathbb{J}_{\mathrm{th}}^{(\omega, l)}\right\}_{k}:=\left|\Lambda_{l}\right|^{-1} \sum_{x \in \Lambda_{l}} \varrho^{(\beta, \omega, \lambda)}\left(I_{\left(x+e_{k}, x\right)}\right) .
$$

(p) The paramagnetic current density is the map

$$
t \mapsto \mathbb{J}_{\mathrm{p}}^{\left(\omega, \eta \overline{\mathbf{A}}_{l}\right)}(t) \equiv \mathbb{J}_{\mathrm{p}}^{\left(\beta, \omega, \lambda, \eta \overline{\mathbf{A}}_{l}\right)}(t) \in \mathbb{R}^{d}
$$

defined by the space average of the current increment vector inside the box $\Lambda_{l}$ at times $t \geq t_{0}$, that is for any $k \in\{1, \ldots, d\}$,

$$
\left\{\mathbb{J}_{\mathrm{p}}^{\left(\omega, \eta \overline{\mathbf{A}}_{l}\right)}(t)\right\}_{k}:=\left|\Lambda_{l}\right|^{-1} \sum_{x \in \Lambda_{l}} \rho_{t}^{\left(\beta, \omega, \lambda, \eta \overline{\mathbf{A}}_{l}\right)}\left(I_{\left(x+e_{k}, x\right)}\right)-\varrho^{(\beta, \omega, \lambda)}\left(I_{\left(x+e_{k}, x\right)}\right) .
$$

(d) The diamagnetic (or ballistic) current density

$$
t \mapsto \mathbb{J}_{\mathrm{d}}^{\left(\omega, \eta \overline{\mathbf{A}}_{l}\right)}(t) \equiv \mathbb{J}_{\mathrm{d}}^{\left(\beta, \omega, \lambda, \eta \overline{\mathbf{A}}_{l}\right)}(t) \in \mathbb{R}^{d}
$$

is defined analogously, for any $t \geq t_{0}$ and $k \in\{1, \ldots, d\}$, by

$$
\left\{\mathbb{J}_{\mathrm{d}}^{\left(\omega, \eta \overline{\mathbf{A}}_{l}\right)}(t)\right\}_{k}:=\left|\Lambda_{l}\right|^{-1} \sum_{x \in \Lambda_{l}} \rho_{t}^{\left(\beta, \omega, \lambda, \eta \overline{\mathbf{A}}_{l}\right)}\left(\mathrm{I}_{\left(x+e_{k}, x\right)}^{\eta \overline{\mathbf{A}}_{l}}\right) .
$$

Thermal currents are due to the space inhomogeneity of the fermion system for $\lambda \in \mathbb{R}^{+}$. The paramagnetic current density is only related to the change of internal state $\rho_{t}^{(\beta, \omega, \lambda, \mathbf{A})}$ produced by the electromagnetic field. We show in [BPK2, Theorem 4.1] that it carries the paramagnetic energy increment defined in Section 4.1. The diamagnetic current density corresponds to a raw ballistic flow of 
charged particles caused by the electric field. It yields the diamagnetic energy again defined in Section 4.1. Paramagnetic and diamagnetic currents correspond to different physical phenomena. See [BPK2, Sections 3.4-3.5, 4.4] for more details.

In order to compare the objects we study in the present paper with those of $[\mathrm{KLM}]$ we rewrite the current densities in terms of the one-particle Hilbert space $\ell^{2}(\mathfrak{L})$. Indeed, by using the time-evolving symbols $\mathbf{d}_{t}^{\left(\beta, \omega, \lambda, \eta \overline{\mathbf{A}}_{l}\right)} \in \mathcal{B}\left(\ell^{2}(\mathfrak{L})\right)$ of the quasi-free state $\rho_{t}^{\left(\beta, \omega, \lambda, \eta \overline{\mathbf{A}}_{l}\right)}$, the (full) current density on the direction $e_{k}, k \in$ $\{1, \ldots, d\}$, can be seen as a trace on the one-particle Hilbert space $\ell^{2}(\mathfrak{L})$ for every $l \in \mathbb{R}^{+}:$

$$
\begin{aligned}
& \left\{\mathbb{J}_{\mathrm{th}}^{(\omega, l)}+\mathbb{J}_{\mathrm{p}}^{\left(\omega, \eta \overline{\mathbf{A}}_{l}\right)}(t)+\mathbb{J}_{\mathrm{d}}^{\left(\omega, \eta \overline{\mathbf{A}}_{l}\right)}(t)\right\}_{k} \\
= & -\left|\Lambda_{l}\right|^{-1} \operatorname{Tr}_{\ell^{2}(\mathfrak{L})}\left[\mathbf{d}_{t}^{\left(\beta, \omega, \lambda, \eta \overline{\mathbf{A}}_{l}\right)} \mathrm{P}_{l} i\left[\Delta_{\mathrm{d}}^{\left(\eta \overline{\mathbf{A}}_{l}\right)}, X_{k}\right] \mathrm{P}_{l}\right]+\mathcal{O}\left(l^{-1}\right),
\end{aligned}
$$

uniformly w.r.t. all parameters. Here, for any $l \in \mathbb{R}^{+}, \mathrm{P}_{l} \in \mathcal{B}\left(\ell^{2}(\mathfrak{L})\right)$ is the orthogonal projector with range $\operatorname{lin}\left\{\mathfrak{e}_{x}: x \in \Lambda_{l}\right\}$, i.e., the multiplication operator with the characteristic function of the box $\Lambda_{l}$. For any $k \in\{1, \ldots, d\}, X_{k}$ is the (unbounded) multiplication operator on $\ell^{2}(\mathfrak{L})$ with the $k^{\text {th }}$ space component:

$$
X_{k}(\psi)\left(x_{1}, \ldots, x_{d}\right):=x_{k} \psi\left(x_{1}, \ldots, x_{d}\right)
$$

for all $\psi \in \ell^{2}(\mathfrak{L})$ in the domain of definition of $X_{k}$. The term of order $\mathcal{O}\left(l^{-1}\right)$ in (38) results from the existence of $\mathcal{O}\left(l^{d-1}\right)$ points $x \in \Lambda_{l}$ such that $x+e_{k} \notin \Lambda_{l}$. Therefore, by (38), the full current density can be seen as a kind of density of trace of a velocity operator on the one-particle space $\ell^{2}(\mathfrak{L})$ like in [KLM, Eq. (2.6)]. However, as compared with [KLM, KM], the density operator $\mathbf{d}_{t}^{\left(\beta, \omega, \lambda, \eta \overline{\mathbf{A}}_{l}\right)}$ depends on the size of the box in our formulation.

By [BPK2, Theorem 3.3], the current density behaves, at small $|\eta|$ and uniformly w.r.t. the size of the box, linearly w.r.t. the parameter $\eta$ : For any $\vec{w} \in \mathbb{R}^{d}$ and $\mathcal{A} \in C_{0}^{\infty}(\mathbb{R} ; \mathbb{R})$, there is a strictly positive number $\eta_{0} \in \mathbb{R}^{+}$such that, for $|\eta| \in\left[0, \eta_{0}\right]$,

$$
\begin{array}{lll}
\mathbb{J}_{\mathrm{p}}^{\left(\omega, \eta \overline{\mathbf{A}}_{l}\right)}(t)=\eta J_{\mathrm{p}, l}^{(\omega, \mathcal{A})}(t)+\mathcal{O}\left(\eta^{2}\right), & J_{\mathrm{p}, l}^{(\omega, \mathcal{A})}(t)=\mathcal{O}(1), \\
\mathbb{J}_{\mathrm{d}}^{\left(\omega, \eta \overline{\mathbf{A}}_{l}\right)}(t)=\eta J_{\mathrm{d}, l}^{(\omega, \mathcal{A})}(t)+\mathcal{O}\left(\eta^{2}\right), & J_{\mathrm{d}, l}^{(\omega, \mathcal{A})}(t)=\mathcal{O}(1),
\end{array}
$$

uniformly for $l, \beta \in \mathbb{R}^{+}, \omega \in \Omega, \lambda \in \mathbb{R}_{0}^{+}$and $t \geq t_{0}$. 
The $\mathbb{R}^{d}$-valued linear coefficients

$$
J_{\mathrm{p}, l}^{(\omega, \mathcal{A})} \equiv J_{\mathrm{p}, l}^{(\beta, \omega, \lambda, \vec{w}, \mathcal{A})} \quad \text { and } \quad J_{\mathrm{d}, l}^{(\omega, \mathcal{A})} \equiv J_{\mathrm{d}, l}^{(\beta, \omega, \lambda, \vec{w}, \mathcal{A})}
$$

of the paramagnetic and diamagnetic current densities, respectively, become deterministic for large boxes. They are directly related to the charge transport coefficients $\boldsymbol{\Xi}_{\mathrm{p}}$ and $\boldsymbol{\Xi}_{\mathrm{d}}$ via Ohm's law:

\section{Theorem 3.4 (Classical Ohm's law)}

Let $\beta \in \mathbb{R}^{+}$and $\lambda \in \mathbb{R}_{0}^{+}$. Then, there is a measurable subset $\tilde{\Omega} \equiv \tilde{\Omega}^{(\beta, \lambda)} \subset \Omega$ of full measure such that, for any $\omega \in \tilde{\Omega}, \vec{w} \in \mathbb{R}^{d}, \mathcal{A} \in C_{0}^{\infty}(\mathbb{R} ; \mathbb{R})$ and $t \geq t_{0}$, the following assertions hold true:

(th) Thermal current density:

$$
\lim _{l \rightarrow \infty} \mathbb{J}_{\text {th }}^{(\omega, l)}=(0, \ldots, 0) .
$$

(p) Paramagnetic current density:

$$
\lim _{l \rightarrow \infty} J_{\mathrm{p}, l}^{(\omega, \mathcal{A})}(t)=\lim _{l \rightarrow \infty}\left(\left.\partial_{\eta} \mathbb{J}_{\mathrm{p}}^{\left(\omega, \eta \overline{\mathbf{A}}_{l}\right)}(t)\right|_{\eta=0}\right)=\int_{t_{0}}^{t}\left(\boldsymbol{\Xi}_{\mathrm{p}}(t-s) \vec{w}\right) \mathcal{E}_{s} \mathrm{~d} s .
$$

(d) Diamagnetic current density:

$$
\lim _{l \rightarrow \infty} J_{\mathrm{d}, l}^{(\omega, \mathcal{A})}(t)=\lim _{l \rightarrow \infty}\left(\left.\partial_{\eta} \mathbb{J}_{\mathrm{d}}^{\left(\omega, \eta \overline{\mathbf{A}}_{l}\right)}(t)\right|_{\eta=0}\right)=\left(\boldsymbol{\Xi}_{\mathrm{d}} \vec{w}\right) \int_{t_{0}}^{t} \mathcal{E}_{s} \mathrm{~d} s .
$$

Proof: (th) is Corollary 5.7 (th). Assertions (p) and (d) are deduced from Theorem 3.1, [BPK2, Eqs (44)-(45)] and Lebesgue's dominated convergence theorem. Note that any countable intersection of measurable sets of full measure has full measure.

Exactly like [BPK2, Theorem 3.3], Theorem 3.4 can be extended to macroscopically space-inhomogeneous electromagnetic fields, that is, for all space-rescaled vector potentials $\mathbf{A}_{l}$ (17) with $\mathbf{A} \in \mathbf{C}_{0}^{\infty}$, by exactly the same methods as in the proof of Theorem 4.1. We refrain from doing it at this point, for technical simplicity. Such a result can indeed be deduced from Theorem 4.1, see Equations (61)-(62).

Therefore, for any $\beta \in \mathbb{R}^{+}, \lambda \in \mathbb{R}_{0}^{+}$and $\mathcal{A} \in C_{0}^{\infty}(\mathbb{R} ; \mathbb{R})$, the full current density linear response $J_{\text {lin }} \equiv J_{\text {lin }}^{(\beta, \lambda, \mathcal{A})}$ of the infinite volume fermion system equals

$$
J_{\text {lin }}(t)=\int_{-\infty}^{t}(\Sigma(t-s) \vec{w}) \mathcal{E}_{s} \mathrm{~d} s, \quad t \in \mathbb{R} .
$$


Moreover, $J_{\text {lin }}$ is the sum of paramagnetic and diamagnetic current densities. Such a decomposition of the current is well-known in theoretical physics, see, e.g., [GV, Eq. (A2.14)]. For a discussion on the physical meaning of the paramagnetic and diamagnetic components of the current, in particular in which concerns heat production, we refere to [BPK2, Section 3.5].

\subsection{Green-Kubo Relations and CCR Algebra of Current Fluc- tuations}

For every (lattice translation) $x \in \mathfrak{L}$, the condition

$$
\chi_{x}\left(a_{y}\right)=a_{y+x}, \quad y \in \mathfrak{L},
$$

uniquely defines a $*$-automorphism $\chi_{x}$ of the CAR $C^{*}$-algebra $\mathcal{U}$. For any $l \in \mathbb{R}^{+}$ and $B \in \mathcal{U}$, set

$$
\mathbb{F}^{(l)}(B):=\frac{1}{\left|\Lambda_{l}\right|^{1 / 2}} \sum_{x \in \Lambda_{l}}\left\{\chi_{x}(B)-\varrho^{(\beta, \omega, \lambda)}\left(\chi_{x}(B)\right) \mathbf{1}\right\} .
$$

We name it the fluctuation observable of the element $B \in \mathcal{U}$.

By Theorem 3.1 and Lebesgue's dominated convergence theorem together with Equations (29), (31) and (33), one obtains Green-Kubo relations: For any $\beta \in \mathbb{R}^{+}, \lambda \in \mathbb{R}_{0}^{+}, t \in \mathbb{R}$ and $k, q \in\{1, \ldots, d\}$,

$$
\left\{\boldsymbol{\Xi}_{\mathrm{p}}(t)\right\}_{k, q}=\int_{0}^{t} \lim _{l \rightarrow \infty} \mathbb{E}\left[\varrho^{(\beta, \omega, \lambda)}\left(i\left[\mathbb{F}^{(l)}\left(I_{\left(e_{k}, 0\right)}\right), \mathbb{F}^{(l)}\left(\tau_{s}^{(\omega, \lambda)}\left(I_{\left(e_{q}, 0\right)}\right)\right)\right]\right)\right] \mathrm{d} s
$$

with the current observable $I_{(x, y)}$ defined by (26). See also [BPK2, Eq. (46)] and Theorem 3.5 which ensures the existence of the limit integrand. It is therefore natural to introduce the so-called CCR $C^{*}$-algebra of current fluctuations, which is reminiscent of non-commutative central limit theorems (see, e.g., [GVV]).

To this end, we define the linear subspace

$$
\mathcal{I}:=\operatorname{lin}\left\{\operatorname{Im}\left(a^{*}\left(\psi_{1}\right) a\left(\psi_{2}\right)\right): \psi_{1}, \psi_{2} \in \ell^{1}(\mathfrak{L}) \subset \ell^{2}(\mathfrak{L})\right\} \subset \mathcal{U},
$$

which is the linear hull (lin) of short range bond currents. As explained in [BPK2, Section 5.1.2], it is an invariant subspace of the one-parameter group $\tau^{(\omega, \lambda)}$ for any $\omega \in \Omega$ and $\lambda \in \mathbb{R}_{0}^{+}$. We define from $\mathcal{I}$ a pre-Hilbert space $\check{\mathcal{H}}_{\mathrm{fl}}$ of current fluctuations by using the following positive sesquilinear form $\langle\cdot, \cdot\rangle_{\mathcal{I}}$ : 


\section{Theorem 3.5 (Positive sesquilinear form from current fluctuations)}

Let $\beta \in \mathbb{R}^{+}$and $\lambda \in \mathbb{R}_{0}^{+}$. Then, there is a measurable set $\tilde{\Omega} \equiv \tilde{\Omega}^{(\beta, \lambda)} \subset \Omega$ of full measure such that, for any $\omega \in \tilde{\Omega}$, the limit

$$
\left\langle I, I^{\prime}\right\rangle_{\mathcal{I}}=\lim _{l \rightarrow \infty} \varrho^{(\beta, \omega, \lambda)}\left(\mathbb{F}^{(l)}(I)^{*} \mathbb{F}^{(l)}\left(I^{\prime}\right)\right), \quad I, I^{\prime} \in \mathcal{I}
$$

exists and does not depend on $\omega \in \tilde{\Omega}$.

Proof: $\quad$ See Theorem 5.26.

Because of the Cauchy-Schwarz inequality for $\langle\cdot, \cdot\rangle_{\mathcal{I}}$, the set

$$
\mathcal{I}_{0}:=\left\{I \in \mathcal{I}:\langle I, I\rangle_{\mathcal{I}}=0\right\}
$$

is a subspace of $\mathcal{I}$ and the quotient $\check{\mathcal{H}}_{\mathrm{fl}}:=\mathcal{I} / \mathcal{I}_{0}$ is a pre-Hilbert space w.r.t. to the (well-defined) scalar product

$$
\left\langle[I],\left[I^{\prime}\right]\right\rangle_{\check{\mathcal{H}}_{\mathrm{fl}}}:=\left\langle I, I^{\prime}\right\rangle_{\mathcal{I}}, \quad[I],\left[I^{\prime}\right] \in \check{\mathcal{H}}_{\mathrm{fl}} .
$$

Note that $\mathcal{I}_{0} \neq \mathcal{I}$, in general. Observe also that $\mathcal{I}_{0}$ is an invariant subspace of $\tau^{(\omega, \lambda)}$ because of (21). In particular, for any $t \in \mathbb{R}$,

$$
\left[\tau_{t}^{(\omega, \lambda)}(I)\right]=\left[\tau_{t}^{(\omega, \lambda)}\left(I^{\prime}\right)\right], \quad I, I^{\prime} \in[I] \in \check{\mathcal{H}}_{\mathrm{fl}} .
$$

Therefore, by Theorem 5.27, the dynamics defined by $\tau^{(\omega, \lambda)}$ on $\mathcal{U}$ induces a unitary time evolution on the Hilbert space $\mathcal{H}_{\mathrm{f}}$, the completion of $\check{\mathcal{H}}_{\mathrm{fl}}$ w.r.t. the scalar product $\langle\cdot, \cdot\rangle_{\check{\mathcal{H}}_{\mathrm{f}}}$ : Let $\beta \in \mathbb{R}^{+}$and $\lambda \in \mathbb{R}_{0}^{+}$. Then, there is a measurable set $\tilde{\Omega} \equiv \tilde{\Omega}^{(\beta, \lambda)} \subset \Omega$ of full measure such that, for any $\omega \in \tilde{\Omega}$, there is a unique strongly continuous one-parameter unitary group $\left\{\mathrm{V}_{t}^{(\omega, \lambda)}\right\}_{t \in \mathbb{R}}$ on the Hilbert space $\mathcal{H}_{\mathrm{fl}}$ obeying, for any $t \in \mathbb{R}$,

$$
\mathrm{V}_{t}^{(\omega, \lambda)}([I])=\left[\tau_{t}^{(\omega, \lambda)}(I)\right], \quad[I] \in \check{\mathcal{H}}_{\mathrm{fl}} .
$$

In Section 5.5.3 another construction is considered for an one-parameter unitary group $\left\{\overline{\mathrm{V}}_{t}^{(\lambda)}\right\}_{t \in \mathbb{R}}$ that has similar properties but does not depend on $\omega$. It is not given here for the sake of technical simplicity.

We define next a non-degenerate symplectic bilinear form $\mathfrak{s} \equiv \mathfrak{s}^{(\beta, \lambda)}$ on $\check{\mathcal{H}}_{\mathrm{fl}}$ (seen as a real vector space) by

$$
\mathfrak{s}\left([I],\left[I^{\prime}\right]\right):=\operatorname{Im}\left\langle[I],\left[I^{\prime}\right]\right\rangle_{\check{\mathcal{H}}_{\mathrm{fl}}}, \quad[I],\left[I^{\prime}\right] \in \check{\mathcal{H}}_{\mathrm{fl}} .
$$


Let

$$
\mathcal{W} \equiv \mathcal{W}^{(\beta, \lambda)} \equiv \mathcal{W}\left(\check{\mathcal{H}}_{\mathrm{fl}}, \mathfrak{s}\right)
$$

be the CCR algebra over the symplectic space $\left(\check{\mathcal{H}}_{\mathrm{f}}, \mathfrak{s}\right)$, i.e., the $C^{*}$-algebra generated by the Weyl operators $\mathbf{W}([I]),[I] \in \check{\mathcal{H}}_{\mathrm{f}}$, fulfilling (the Weyl form of) the canonical commutation relations

$$
\mathbf{W}([I]) \mathbf{W}\left(\left[I^{\prime}\right]\right)=\mathrm{e}^{-\frac{i}{2} \mathfrak{s}\left([I],\left[I^{\prime}\right]\right)} \mathbf{W}\left([I]+\left[I^{\prime}\right]\right), \quad[I],\left[I^{\prime}\right] \in \check{\mathcal{H}}_{\mathrm{fl}} .
$$

See, e.g., [BR2, Section 5.2.2.2.] for more details. Because of Remark 3.7 we name the space $\mathcal{W}$ the algebra of current fluctuations of the system at inverse temperature $\beta \in \mathbb{R}^{+}$and strength $\lambda \in \mathbb{R}_{0}^{+}$of disorder.

Take any regular state $\rho$ on the $C^{*}$-algebra $\mathcal{W}$. An example of such a state is the so-called Fock state, uniquely defined by

$$
\varrho_{\text {Fock }}^{(\beta, \lambda)}(\mathbf{W}([I]))=G_{\text {Fock }}^{(\beta, \lambda)}([I]):=\mathrm{e}^{-\frac{1}{4}\|[I]\|_{\check{\mathcal{H}}_{\mathrm{fl}}}^{2}}, \quad[I] \in \check{\mathcal{H}}_{\mathrm{fl}},
$$

see [BR2, Section 5.2.3.]. Let $\left(\mathfrak{H}_{\rho}, \pi_{\rho}, \Psi_{\rho}\right)$ be the GNS representation of $\mathcal{W}$ w.r.t. the state $\rho$. Then, for any $[I] \in \breve{\mathcal{H}}_{\mathrm{fl}}$, there exists a Bose field $\Phi([I])$ - a self-adjoint operator affiliated with the von Neumann algebra $\pi_{\rho}(\mathcal{W})^{\prime \prime}$ - such that

$$
\pi_{\rho}(\mathbf{W}([I]))=\exp (i \Phi([I])), \quad[I] \in \check{\mathcal{H}}_{\mathrm{fl}} .
$$

Assume additionally that

$$
\Psi_{\rho} \in \operatorname{Dom}\left(\Phi([I])^{\infty}\right), \quad[I] \in \check{\mathcal{H}}_{\mathrm{fl}} .
$$

This assumption is for instance satisfied by the Fock state $\varrho_{\text {Fock }}^{(\beta, \lambda)}$.

Now, by Equations (41) and (43)-(44) together with Theorem 3.5, observe that

$$
\left\{\boldsymbol{\Xi}_{\mathrm{p}}(t)\right\}_{k, q}=-2 \mathfrak{s}\left(\left[I_{e_{k}, 0}\right], \int_{0}^{t} \mathrm{~V}_{s}^{(\omega, \lambda)}\left(\left[I_{e_{q}, 0}\right]\right) \mathrm{d} s\right)
$$

for any $\omega \in \tilde{\Omega}, t \in \mathbb{R}$ and $k, q \in\{1, \ldots, d\}$. Using this last assertion together with (45)-(47) we then arrive at the equality

$$
\left\{\boldsymbol{\Xi}_{\mathrm{p}}(t)\right\}_{k, q}=-4 \operatorname{Im}\left\langle\Psi_{\rho}, \Phi\left(\left[I_{e_{k}, 0}\right]\right) \Phi\left(\int_{0}^{t} \mathrm{~V}_{s}^{(\omega, \lambda)}\left(\left[I_{e_{q}, 0}\right]\right) \mathrm{d} s\right) \Psi_{\rho}\right\rangle_{\mathfrak{H}_{\rho}}
$$

for $\omega \in \tilde{\Omega}, t \in \mathbb{R}$ and all $k, q \in\{1, \ldots, d\}$. In particular, by Theorem 3.4 (p), we can rewrite the Green-Kubo relations for the paramagnetic current density at any time in terms of time-correlations of the Bose fields $\Phi$ defined above: 


\section{Theorem 3.6 (Green-Kubo relations)}

Let $\beta \in \mathbb{R}^{+}$and $\lambda \in \mathbb{R}_{0}^{+}$. Then, there is a measurable set $\tilde{\Omega} \equiv \tilde{\Omega}^{(\beta, \lambda)} \subset \Omega$ of full measure such that, for any $\vec{w}:=\left(w_{1}, \ldots, w_{d}\right) \in \mathbb{R}^{d}, \mathcal{A} \in C_{0}^{\infty}(\mathbb{R} ; \mathbb{R}), t \geq t_{0}$, $\omega \in \tilde{\Omega}$ and $k \in\{1, \ldots, d\}$,

$$
\begin{aligned}
& \lim _{l \rightarrow \infty}\left\{J_{\mathrm{p}, l}^{(\omega, \mathcal{A})}(t)\right\}_{k} \\
= & -4 \operatorname{Im} \int_{t_{0}}^{t} \sum_{q=1}^{d}\left\langle\Psi_{\rho}, \Phi\left(\left[I_{e_{k}, 0}\right]\right) \Phi\left(\int_{0}^{t-s_{1}} \mathrm{~V}_{s_{2}}^{(\omega, \lambda)}\left(\left[I_{e_{q}, 0}\right]\right) \mathrm{d} s_{2}\right) \Psi_{\rho}\right\rangle_{\mathfrak{H}_{\rho}} w_{q} \mathcal{E}_{s_{1}} \mathrm{~d} s_{1} .
\end{aligned}
$$

By using additionally the i.i.d. property of the random variables, one can simplify this last equation to obtain, almost surely, that

$$
\begin{aligned}
& \lim _{l \rightarrow \infty} J_{\mathrm{p}, l}^{(\omega, \mathcal{A})}(t) \\
= & -4 \vec{w} \operatorname{Im} \int_{t_{0}}^{t}\left\langle\Psi_{\rho}, \Phi\left(\left[I_{e_{1}, 0}\right]\right) \Phi\left(\int_{0}^{t-s_{1}} \mathrm{~V}_{s_{2}}^{(\omega, \lambda)}\left(\left[I_{e_{1}, 0}\right]\right) \mathrm{d} s_{2}\right) \Psi_{\rho}\right\rangle_{\mathfrak{H}_{\rho}} \mathcal{E}_{s_{1}} \mathrm{~d} s_{1} .
\end{aligned}
$$

See Equation (67) below.

\section{Remark 3.7 (Non-commutative central limit theorems)}

By analogy with previous results on non-commutative central limit theorems (see, e.g., [GVV]), we conjecture that the generating functional

$$
G_{\mathrm{fl}}^{(\beta, \lambda)}([I]):=\lim _{l \rightarrow \infty} \varrho^{(\beta, \omega, \lambda)}\left(\exp \left(i \mathbb{F}^{(l)}(I)\right)\right), \quad[I] \in \check{\mathcal{H}}_{\mathrm{fl}},
$$

exists for all $\omega$ in a set $\tilde{\Omega} \subset \Omega$ of full measure, is independent of $\omega$ in this set, and uniquely defines a quasi-free state $\varrho_{\mathrm{fl}}^{(\beta, \lambda)}$ on $\mathcal{W}$ with

$$
\varrho_{\mathrm{fl}}^{(\beta, \lambda)}(\mathbf{W}([I]))=G_{\mathrm{fl}}^{(\beta, \lambda)}([I]), \quad[I] \in \check{\mathcal{H}}_{\mathrm{fl}} .
$$

From the physical point of view, the natural choice of the regular state in Theorem 3.6 should be $\varrho_{\mathrm{fl}}^{(\beta, \lambda)}$.

\section{AC-Conductivity Measure From Joule's Law}

There is however one important property of the equilibrium states which is related to the concept of the work. Namely for such states $L^{h}(\omega) \geqq 0$ [i.e., the energy 
transmitted to the system is positive, ] provided the final external conditions coincide with the original ones: $h_{T}=0$. This fact is strongly related to the second principle of thermodynamics saying that systems in the equilibrium are unable to perform mechanical work in cyclic processes. We describe this property saying that the equilibrium states are passive.

[Pusz-Woronowicz, 1978]

As discussed in the Introduction, our derivation of an AC-conductivity measure is based on the second principle of thermodynamics in the above form (cf. [PW, p. 276]). It dovetails with the positivity of the heat production for cyclic processes on equilibrium states. Indeed, we analyze in [BPK1] the heat production of the fermion system considered here by using Araki's relative entropy for states of infinitely extended systems. We verify in particular the first law of thermodynamics by identifying the heat production with an energy increment defined below, see [BPK1, Theorem 3.2]. As originally observed by J. P. Joule, in conducting media, electric energy is always lost in form of heat. This is (part of) the celebrated Joule's law of (classical) electricity theory. It corresponds to the passivity of equilibrium states, which is proven for KMS states in [PW, Theorem 1.2]. It follows from Joule's law that the in-phase paramagnetic conductivity is the kernel of a positive quadratic form on the space of smooth, compactly supported functions satisfying an AC-condition. Recall that the latter is related to cyclic electromagnetic processes. Together with the Bochner-Schwartz theorem [RS2, Theorem IX.10], it in turn yields the existence of the AC-conductivity measure.

We thus need to prove Joule's law, as it is done for microscopic electric fields in [BPK2, Theorem 4.1]. It is an important result of this paper because we seek to get a rigorous microscopic description of the phenomenon of linear conductivity from basic principles of thermodynamics and quantum mechanics, only. To this end we start by introducing energy densities, in particular the heat production density.

\subsection{Energy Densities}

For any $L \in \mathbb{R}^{+}$, the internal energy observable in the box $\Lambda_{L}(25)$ is defined by

$$
H_{L}^{(\omega, \lambda)}:=\sum_{x, y \in \Lambda_{L}}\left\langle\mathfrak{e}_{x},\left(\Delta_{\mathrm{d}}+\lambda V_{\omega}\right) \mathfrak{e}_{y}\right\rangle a_{x}^{*} a_{y} \in \mathcal{U}
$$


When the electromagnetic field is switched on, i.e., for $t \geq t_{0}$, the (time-dependent) total energy observable in the box $\Lambda_{L}$ is then equal to $H_{L}^{(\omega, \lambda)}+W_{t}^{\mathbf{A}}$, where, for any $\mathbf{A} \in \mathbf{C}_{0}^{\infty}$ and $t \in \mathbb{R}$,

$$
W_{t}^{\mathbf{A}}:=\sum_{x, y \in \Lambda_{L}}\left\langle\mathfrak{e}_{x},\left(\Delta_{\mathrm{d}}^{(\mathbf{A})}-\Delta_{\mathrm{d}}\right) \mathfrak{e}_{y}\right\rangle a_{x}^{*} a_{y} \in \mathcal{U}
$$

is the electromagnetic potential energy observable.

Like in [BPK2, Section 4], we now define below four sorts of energy associated with the fermion system for any $\beta \in \mathbb{R}^{+}, \omega \in \Omega, \lambda \in \mathbb{R}_{0}^{+}$and $\mathbf{A} \in \mathbf{C}_{0}^{\infty}$ :

(Q) The internal energy increment $\mathbf{S}^{(\omega, \mathbf{A})} \equiv \mathbf{S}^{(\beta, \omega, \lambda, \mathbf{A})}$ is a map from $\mathbb{R}$ to $\mathbb{R}_{0}^{+}$ defined by

$$
\mathbf{S}^{(\omega, \mathbf{A})}(t):=\lim _{L \rightarrow \infty}\left\{\rho_{t}^{(\beta, \omega, \lambda, \mathbf{A})}\left(H_{L}^{(\omega, \lambda)}\right)-\varrho^{(\beta, \omega, \lambda)}\left(H_{L}^{(\omega, \lambda)}\right)\right\} .
$$

It takes positive finite values because of [BPK1, Theorem 3.2].

(P) The electromagnetic potential energy (increment) $\mathbf{P}^{(\omega, \mathbf{A})} \equiv \mathbf{P}^{(\beta, \omega, \lambda, \mathbf{A})}$ is a map from $\mathbb{R}$ to $\mathbb{R}$ defined by

$$
\mathbf{P}^{(\omega, \mathbf{A})}(t):=\rho_{t}^{(\beta, \omega, \lambda, \mathbf{A})}\left(W_{t}^{\mathbf{A}}\right)=\rho_{t}^{(\beta, \omega, \lambda, \mathbf{A})}\left(W_{t}^{\mathbf{A}}\right)-\varrho^{(\beta, \omega, \lambda)}\left(W_{t_{0}}^{\mathbf{A}}\right) .
$$

(p) The paramagnetic energy increment $\mathfrak{J}_{\mathrm{p}}^{(\omega, \mathbf{A})} \equiv \mathfrak{I}_{\mathrm{p}}^{(\beta, \omega, \lambda, \mathbf{A})}$ is the map from $\mathbb{R}$ to $\mathbb{R}$ defined by

$$
\mathfrak{I}_{\mathrm{p}}^{(\omega, \mathbf{A})}(t):=\lim _{L \rightarrow \infty}\left\{\rho_{t}^{(\beta, \omega, \lambda, \mathbf{A})}\left(H_{L}^{(\omega, \lambda)}+W_{t}^{\mathbf{A}}\right)-\varrho^{(\beta, \omega, \lambda)}\left(H_{L}^{(\omega, \lambda)}+W_{t}^{\mathbf{A}}\right)\right\} .
$$

(d) The diamagnetic energy (increment) $\mathfrak{I}_{\mathrm{d}}^{(\omega, \mathbf{A})} \equiv \mathfrak{I}_{\mathrm{d}}^{(\beta, \omega, \lambda, \mathbf{A})}$ is the map from $\mathbb{R}$ to $\mathbb{R}$ defined by

$$
\Im_{\mathrm{d}}^{(\omega, \mathbf{A})}(t):=\varrho^{(\beta, \omega, \lambda)}\left(W_{t}^{\mathbf{A}}\right)=\varrho^{(\beta, \omega, \lambda)}\left(W_{t}^{\mathbf{A}}\right)-\varrho^{(\beta, \omega, \lambda)}\left(W_{t_{0}}^{\mathbf{A}}\right) .
$$

In other words, $\mathbf{P}^{(\omega, \mathbf{A})}$ and $\mathfrak{I}_{\mathrm{d}}^{(\omega, \mathbf{A})}$ are the electromagnetic potential energy of the fermion system in the internal state $\rho_{t}^{(\beta, \omega, \lambda, \mathbf{A})}$ and thermal equilibrium state $\varrho^{(\beta, \omega, \lambda)}$, respectively. $\mathbf{S}^{(\omega, \mathbf{A})}$ represents the increase of internal energy, while $\mathfrak{J}_{\mathrm{p}}^{(\omega, \mathbf{A})}$ is the part of electromagnetic work implying a change of the internal state of the system. 
Note that the limits (51) and (53) exist at all times because the increase of total energy of the infinite system equals

$$
\mathbf{S}^{(\omega, \mathbf{A})}(t)+\mathbf{P}^{(\omega, \mathbf{A})}(t)=\mathfrak{I}_{\mathrm{p}}^{(\omega, \mathbf{A})}(t)+\mathfrak{I}_{\mathrm{d}}^{(\omega, \mathbf{A})}(t) .
$$

The increase of total energy is shown in [BPK1, Theorem 5.8] to be the work performed by the electric field. See also [BPK2, Sections 4.2-4.3] for more details.

By using the time-evolving symbols $\mathbf{d}_{t}^{(\beta, \omega, \lambda, \mathbf{A})} \in \mathcal{B}\left(\ell^{2}(\mathfrak{L})\right)$, all energy increments can be seen as limits of traces on the one-particle Hilbert space $\ell^{2}(\mathfrak{L})$. For instance,

$$
\mathfrak{I}_{\mathrm{p}}^{(\omega, \mathbf{A})}(t)=\lim _{L \rightarrow \infty} \operatorname{Tr}_{\ell^{2}(\mathfrak{L})}\left[\left(\mathbf{d}_{t}^{(\beta, \omega, \lambda, \mathbf{A})}-\mathbf{d}_{t_{0}}^{(\beta, \omega, \lambda, \mathbf{A})}\right) \mathrm{P}_{L}\left(\Delta_{\mathrm{d}}^{(\mathbf{A})}+\lambda V_{\omega}\right) \mathrm{P}_{L}\right],
$$

where, for any $L \in \mathbb{R}^{+}, \mathrm{P}_{L} \in \mathcal{B}\left(\ell^{2}(\mathfrak{L})\right)$ is the orthogonal projector with range $\operatorname{lin}\left\{\mathfrak{e}_{x}: x \in \Lambda_{L}\right\}$, i.e., the multiplication operator with the characteristic function of the box $\Lambda_{L}$.

Observe that the energies

$$
\mathbf{P}^{\left(\omega, \eta \mathbf{A}_{l}\right)}, \quad \mathfrak{I}_{\mathrm{p}}^{\left(\omega, \eta \mathbf{A}_{l}\right)}(t), \quad \mathfrak{I}_{\mathrm{d}}^{\left(\omega, \eta \mathbf{A}_{l}\right)}(t),
$$

are all of order $\mathcal{O}\left(\eta^{2} l^{d}\right)$, by [BPK2, Theorem 4.1]. This can physically be understood by the fact that, by classical electrodynamics, the energy carried by electromagnetic fields are proportional to their $L^{2}$-norms. These are also of order $\mathcal{O}\left(\eta^{2} l^{d}\right)$ in the case of a potential of the form $\eta \mathbf{A}_{l}$. As a consequence, for any $\beta \in \mathbb{R}^{+}, \omega \in \Omega, \lambda \in \mathbb{R}_{0}^{+}$and $\mathbf{A} \in \mathbf{C}_{0}^{\infty}$, we define four energy densities:

(Q) The heat production (or internal energy) density $\mathbf{S} \equiv \mathbf{s}^{(\beta, \omega, \lambda, \mathbf{A})}$ is a map from $\mathbb{R}$ to $\mathbb{R}_{0}^{+}$defined by

$$
\mathbf{s}(t):=\lim _{\left(\eta, l^{-1}\right) \rightarrow(0,0)}\left\{\left(\eta^{2}\left|\Lambda_{l}\right|\right)^{-1} \mathbf{S}^{\left(\omega, \eta \mathbf{A}_{l}\right)}(t)\right\} .
$$

This map has positive finite value because of [BPK1, Theorem 3.2].

(P) The (electromagnetic) potential energy density $\mathbf{p} \equiv \mathbf{p}^{(\beta, \omega, \lambda, \mathbf{A})}$ is a map from $\mathbb{R}$ to $\mathbb{R}$ defined by

$$
\mathbf{p}(t):=\lim _{\eta \rightarrow 0} \lim _{l \rightarrow \infty}\left\{\left(\eta^{2}\left|\Lambda_{l}\right|\right)^{-1} \mathbf{P}^{\left(\omega, \eta \mathbf{A}_{l}\right)}(t)\right\} .
$$


(p) The paramagnetic energy density $\mathfrak{i}_{\mathrm{p}} \equiv \mathfrak{i}_{\mathrm{p}}^{(\beta, \omega, \lambda, \mathbf{A})}$ is a map from $\mathbb{R}$ to $\mathbb{R}$ defined by

$$
\mathfrak{i}_{\mathrm{p}}(t):=\lim _{\left(\eta, l^{-1}\right) \rightarrow(0,0)}\left\{\left(\eta^{2}\left|\Lambda_{l}\right|\right)^{-1} \mathfrak{I}_{\mathrm{p}}^{\left(\omega, \eta \mathbf{A}_{l}\right)}(t)\right\} .
$$

(d) The diamagnetic energy density $\mathfrak{i}_{\mathrm{d}} \equiv \mathfrak{i}_{\mathrm{d}}^{(\beta, \omega, \lambda, \mathbf{A})}$ a map from $\mathbb{R}$ to $\mathbb{R}$ defined by

$$
\mathfrak{i}_{\mathrm{d}}(t):=\lim _{\eta \rightarrow 0} \lim _{l \rightarrow \infty}\left\{\left(\eta^{2}\left|\Lambda_{l}\right|\right)^{-1} \mathfrak{I}_{\mathrm{d}}^{\left(\omega, \eta \mathbf{A}_{l}\right)}(t)\right\} .
$$

For $\omega$ in a measurable subset of full measure, all energy densities above exist and become deterministic:

\subsection{Classical Joule's Law}

Note that the probability measure $\mathfrak{a}_{\Omega}$ defined by (8) is translation invariant. As a consequence, charge transport properties on macroscopic scales are invariant under space translation. By following the heuristics presented in [BPK2, Section 4.4], we deduce from Theorem 3.4 that, for $\beta \in \mathbb{R}^{+}, \lambda \in \mathbb{R}_{0}^{+}$and any electromagnetic potential $\mathbf{A} \in \mathbf{C}_{0}^{\infty}$, the electric field $E_{\mathbf{A}}$ yields paramagnetic and diamagnetic currents linear response coefficients respectively equal to

$$
\begin{aligned}
J_{\mathrm{p}}(t, x) & \equiv J_{\mathrm{p}}^{(\beta, \lambda, \mathbf{A})}(t, x):=\int_{t_{0}}^{t} \boldsymbol{\Xi}_{\mathrm{p}}(t-s) E_{\mathbf{A}}(s, x) \mathrm{d} s, \\
J_{\mathrm{d}}(t, x) & \equiv J_{\mathrm{d}}^{(\beta, \lambda, \mathbf{A})}(t, x):=\boldsymbol{\Xi}_{\mathrm{d}} \int_{t_{0}}^{t} E_{\mathbf{A}}(s, x) \mathrm{d} s
\end{aligned}
$$

at any position $x \in \mathbb{R}^{d}$ and time $t \in \mathbb{R}$.

Therefore, we expect the (density of) work delivered at time $t \geq t_{0}$ by paramagnetic and diamagnetic currents to be equal to

$$
\begin{aligned}
& \int_{\mathbb{R}^{d}} \mathrm{~d}^{d} x \int_{t_{0}}^{t} \mathrm{~d} s\left\langle E_{\mathbf{A}}(s, x), J_{\mathrm{p}}(s, x)\right\rangle \\
= & \int_{\mathbb{R}^{d}} \mathrm{~d}^{d} x \int_{t_{0}}^{t} \mathrm{~d} s_{1} \int_{t_{0}}^{s_{1}} \mathrm{~d} s_{2}\left\langle E_{\mathbf{A}}\left(s_{1}, x\right), \boldsymbol{\Xi}_{\mathrm{p}}\left(s_{1}-s_{2}\right) E_{\mathbf{A}}\left(s_{2}, x\right)\right\rangle
\end{aligned}
$$


and

$$
\begin{aligned}
& \int_{\mathbb{R}^{d}} \mathrm{~d}^{d} x \int_{t_{0}}^{t} \mathrm{~d} s\left\langle E_{\mathbf{A}}(s, x), J_{\mathrm{d}}(s, x)\right\rangle \\
= & \int_{\mathbb{R}^{d}} \mathrm{~d}^{d} x \int_{t_{0}}^{t} \mathrm{~d} s_{1} \int_{t_{0}}^{s_{1}} \mathrm{~d} s_{2}\left\langle E_{\mathbf{A}}\left(s_{1}, x\right), \boldsymbol{\Xi}_{\mathrm{d}} E_{\mathbf{A}}\left(s_{2}, x\right)\right\rangle,
\end{aligned}
$$

respectively.

In contrast to [BPK2, Section 4.4], there is no current density at equilibrium, by Theorem 3.4 (th). Hence, (63) and (64) should be the work density performed by the electromagnetic field. Moreover, since the diamagnetic energy (64) vanishes for $t \geq t_{1}$ when there is not anymore any electric field, (63) should be the heat density $\mathbf{s}(t)$ (57), by the second principle of thermodynamics. We prove this heuristics in Section 5 and obtain the following theorem:

\section{Theorem 4.1 (Classical Joule's law)}

Let $\beta \in \mathbb{R}^{+}$and $\lambda \in \mathbb{R}_{0}^{+}$. Then, there is a measurable subset $\tilde{\Omega} \equiv \tilde{\Omega}^{(\beta, \lambda)} \subset \Omega$ of full measure such that, for any $\omega \in \tilde{\Omega}, \mathbf{A} \in \mathbf{C}_{0}^{\infty}$ and $t \geq t_{0}$ :

(p) Paramagnetic energy density:

$$
\mathfrak{i}_{\mathrm{p}}(t)=\int_{\mathbb{R}^{d}} \mathrm{~d}^{d} x \int_{t_{0}}^{t} \mathrm{~d} s\left\langle E_{\mathbf{A}}(s, x), J_{\mathrm{p}}(s, x)\right\rangle .
$$

(d) Diamagnetic energy density:

$$
\mathfrak{i}_{\mathrm{d}}(t)=\int_{\mathbb{R}^{d}} \mathrm{~d}^{d} x \int_{t_{0}}^{t} \mathrm{~d} s\left\langle E_{\mathbf{A}}(s, x), J_{\mathrm{d}}(s, x)\right\rangle .
$$

(Q) Heat density:

$$
\mathbf{s}(t)=\mathfrak{i}_{\mathrm{p}}(t)-\int_{\mathbb{R}^{d}} \mathrm{~d}^{d} x \int_{t_{0}}^{t} \mathrm{~d} s\left\langle E_{\mathbf{A}}(s, x), J_{\mathrm{p}}(t, x)\right\rangle .
$$

(P) Electromagnetic potential energy density:

$$
\mathbf{p}(t)=\mathfrak{i}_{\mathrm{d}}(t)+\int_{\mathbb{R}^{d}} \mathrm{~d}^{d} x \int_{t_{0}}^{t} \mathrm{~d} s\left\langle E_{\mathbf{A}}(s, x), J_{\mathrm{p}}(t, x)\right\rangle .
$$

Proof: Assertion (p) corresponds to Theorem 5.21 and Lemma 5.22 together with Fubini's theorem, while (d) is Theorem 5.9. Then, $(\mathbf{Q})$ and $(\mathbf{P})$ follow from (55) combined with Theorem 5.24. 
By using Definition 3.2 and Theorem 4.1, for any $\beta \in \mathbb{R}^{+}, \lambda \in \mathbb{R}_{0}^{+}$and $\mathbf{A} \in \mathbf{C}_{0}^{\infty}$, the coefficient

$$
\mathfrak{E}_{\operatorname{lin}}(t) \equiv \mathfrak{E}_{\operatorname{lin}}^{(\beta, \lambda, \mathbf{A})}(t):=\mathfrak{i}_{\mathrm{p}}(t)+\mathfrak{i}_{\mathrm{s}}(t)=\mathbf{s}(t)+\mathbf{p}(t)
$$

corresponding to the total energy density delivered to the system by small electromagnetic fields at time $t \in \mathbb{R}$ is equal to

$$
\mathfrak{E}_{\operatorname{lin}}(t)=\int_{\mathbb{R}^{d}} \mathrm{~d}^{d} x \int_{t_{0}}^{t} \mathrm{~d} s_{1} \int_{t_{0}}^{s_{1}} \mathrm{~d} s_{2}\left\langle E_{\mathbf{A}}\left(s_{1}, x\right), \Sigma\left(s_{1}-s_{2}\right) E_{\mathbf{A}}\left(s_{2}, x\right)\right\rangle .
$$

For any $t \geq t_{1}$, i.e., for cyclic electromagnetic processes, we deduce from [BPK1, Theorem 3.2] that

$$
\mathfrak{i}_{\mathrm{d}}(t)=\mathbf{p}(t)=0 \quad \text { and } \quad \mathfrak{E}_{\text {lin }}(t)=\mathbf{s}(t)=\mathfrak{i}_{\mathrm{p}}(t) \geq 0 .
$$

In other words, when the electromagnetic field is switched off, all the electromagnetic work has been converted into heat, as expected from the second principle of thermodynamics. This phenomenology is related to Joule's law in the AC-regime.

Indeed, the J. P. Joule originally observed that the heat (per second) produced within a circuit is proportional to the electric resistance and the square of the current. There are two clear similarities to the results presented here:

- Like Joule's law, Theorem 4.1 (Q) describes the rate at which resistance in the fermion system converts electric energy into heat energy. This thermal effect is directly related with current fluctuations via Green-Kubo relations, as explained in Section 3.3.

- Quantitatively, Theorem 4.1 is the version of Joule's law, in the DC- and AC-regimes, with currents and resistance replaced by electric fields and (in phase) conductivity.

In fact, the derivation of Joule's law in its original formulation, that is, with currents and resistance rather than electric fields and conductivity, can be performed by using the arguments of [BPK2, Section 4.5]. We omit the details.

In presence of electromagnetic fields, i.e., at times $t \in\left[t_{0}, t_{1}\right]$ for which the AC-condition (13) does not hold, the situation is exactly the one described in [BPK2]: The raw ballistic movement of charged particles, that is responsible for the diamagnetic currents, creates a kind of "propagating wave front" that destabilizes the whole system by changing its internal state. These induce, at their turn, 
paramagnetic currents, by an effect of (quantum) viscosity. The latter (a) modify the potential energy and (b) produce a quantity of entropy (heat) that survives even after turning off the electromagnetic potential. For more detailed discussions on these effects see [BPK2, Section 4.4].

\section{Remark 4.2 (Role of field and space scales)}

Note that the limits $\eta \rightarrow 0$ and $l \rightarrow \infty$ in (58) and (60) do not generally commute for $\lambda \in \mathbb{R}^{+}$. This feature comes from the existence of thermal currents for spaceinhomogeneous media which are order $\mathcal{O}(\eta)$ (an not $\mathcal{O}\left(\eta^{2}\right)$ ), at fixed $l \in \mathbb{R}^{+}$. See [BPK2, Theorem 4.1], (105)-(106) and Theorem 5.8. In this case, the heat production and the paramagnetic energy increment, which are always of order $\mathcal{O}\left(\eta^{2} l^{d}\right)$, are negligible at times $t \in\left(t_{0}, t_{1}\right)$ as compared to the potential and diamagnetic energies, as $\eta \rightarrow 0$. However, for $t \geq t_{1}$, i.e., for cyclic electromagnetic processes, the results of Theorem 4.1 hold true even if one interchanges the limits $\eta \rightarrow 0$ and $l \rightarrow \infty$ in (58) and (60). This is of course coherent with the second principle of thermodynamics and Joule's law in the AC-regime.

\subsection{AC-Conductivity Measure}

Recall that the random variables are independently and identically distributed (i.i.d.), see Section 2.1. As to be expected, this yields scalar paramagnetic charge transport coefficients (Lemma 5.23):

$$
\boldsymbol{\Xi}_{\mathrm{p}}(t)=\boldsymbol{\sigma}_{\mathrm{p}}(t) \operatorname{Id}_{\mathbb{R}^{d}}, \quad t \in \mathbb{R},
$$

where, for any $\beta \in \mathbb{R}^{+}$and $\lambda \in \mathbb{R}_{0}^{+}, \boldsymbol{\sigma}_{\mathrm{p}} \equiv \boldsymbol{\sigma}_{\mathrm{p}}^{(\beta, \lambda)}$ is a well-defined real function. By [BPK2, Corollary 3.2 (i)-(ii) and (iv)] and (67), $\boldsymbol{\sigma}_{\mathrm{p}} \in C\left(\mathbb{R} ; \mathbb{R}_{0}^{-}\right)$and $\boldsymbol{\sigma}_{\mathrm{p}}(t)=$ $\sigma_{\mathrm{p}}(|t|)$ with $\boldsymbol{\sigma}_{\mathrm{p}}(0)=0$. A detailed study of its properties will be performed in the subsequent paper. In the same way, by (32),

$$
\boldsymbol{\Xi}_{\mathrm{d}}=\boldsymbol{\sigma}_{\mathrm{d}} \operatorname{Id}_{\mathbb{R}^{d}}
$$

where, for any $\beta \in \mathbb{R}^{+}$and $\lambda \in \mathbb{R}_{0}^{+}, \boldsymbol{\sigma}_{\mathrm{d}} \equiv \boldsymbol{\sigma}_{\mathrm{d}}^{(\beta, \lambda)}$ is the constant defined by

$$
\boldsymbol{\sigma}_{\mathrm{d}}:=\mathbb{E}\left[\sigma_{\mathrm{d}}^{(\omega)}\left(e_{1}, 0\right)\right] \in[-2,2] .
$$

In fact, one can use the scalar product $\langle\cdot, \cdot\rangle$ in $\ell^{2}(\mathfrak{L})$, the canonical orthonormal basis $\left\{\mathfrak{e}_{x}\right\}_{x \in \mathfrak{L}}$ of $\ell^{2}(\mathfrak{L})$ defined by $\mathfrak{e}_{x}(y) \equiv \delta_{x, y}$, and the symbol $\mathbf{d}_{\text {fermi }}^{(\beta, \omega, \lambda)}$ defined by (23), to get that

$$
\boldsymbol{\sigma}_{\mathrm{d}}=2 \operatorname{Re}\left\{\mathbb{E}\left[\left\langle\mathfrak{e}_{e_{1}}, \mathbf{d}_{\text {fermi }}^{(\beta, \omega, \lambda)} \mathfrak{e}_{0}\right\rangle\right]\right\}
$$


for any $\beta \in \mathbb{R}^{+}$and $\lambda \in \mathbb{R}_{0}^{+}$. By (67)-(68), the (full) conductivity $\Sigma$, which is by Definition 3.2 the sum of the paramagnetic and diamagnetic conductivities, equals

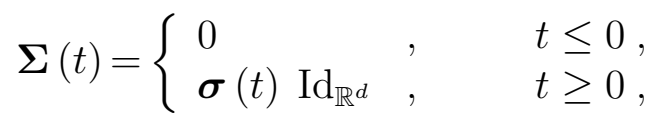

for any $\beta \in \mathbb{R}^{+}, \lambda \in \mathbb{R}_{0}^{+}$and $t \in \mathbb{R}$. Here, the so-called in-phase conductivity $\boldsymbol{\sigma} \equiv \boldsymbol{\sigma}^{(\beta, \lambda)} \in C(\mathbb{R} ; \mathbb{R})$ at $\beta \in \mathbb{R}^{+}$and $\lambda \in \mathbb{R}_{0}^{+}$is the continuous function defined, for any $t \in \mathbb{R}$, by

$$
\boldsymbol{\sigma}(t):=\boldsymbol{\sigma}_{\mathrm{d}}+\boldsymbol{\sigma}_{\mathrm{p}}(t) .
$$

This is a special situation which results from the i.i.d. property of the random variables. For external potentials having non-trivial space correlations the conductivity $\boldsymbol{\Sigma}(t) \in \mathcal{B}\left(\mathbb{R}^{d}\right)$ is, in general, not of the form $\boldsymbol{\sigma}(t) \operatorname{Id}_{\mathbb{R}^{d}}, \boldsymbol{\sigma}(t) \in \mathbb{R}$.

Only the in-phase paramagnetic conductivity $\sigma_{\mathrm{p}}$ is responsible for heat production. In fact, Theorem 4.1 (p) together with the AC-condition (13) uniquely determines the quantity $\boldsymbol{\sigma}_{\mathrm{p}}(t)$ for all $t \in \mathbb{R}$ because $\boldsymbol{\sigma}_{\mathrm{p}}(0)=0$ and $\boldsymbol{\sigma}_{\mathrm{p}}(t)=$ $\sigma_{\mathrm{p}}(|t|)$. To see this more explicitly we consider the following choice of electromagnetic potential $\mathbf{A} \in \mathbf{C}_{0}^{\infty}$ : Take any smooth, compactly supported functions $\mathcal{E} \in C_{0}^{\infty}(\mathbb{R})$ and $\psi \in C_{0}^{\infty}\left(\mathbb{R}^{d} ; \mathbb{R}\right)$ such that

$$
\int_{\mathbb{R}} \mathcal{E}_{t} \mathrm{~d} t=0 \quad \text { and } \quad \int_{\mathbb{R}^{d}} \psi^{2}(x) \mathrm{d}^{d} x=1 .
$$

Next, pick any normalized vector $\vec{w}:=\left(w_{1}, \ldots, w_{d}\right) \in \mathbb{R}^{d}(|\vec{w}|=1)$ and define

$$
\mathbf{A}^{(\mathcal{E}, \psi)}(t, x):=\mathbf{A}_{1}^{(\mathcal{E}, \psi)}(t, x) e_{1}+\cdots+\mathbf{A}_{d}^{(\mathcal{E}, \psi)}(t, x) e_{d}
$$

for all $t \in \mathbb{R}$ and $x \in \mathbb{R}^{d}$, where, for any $k \in\{1, \ldots, d\}$,

$$
\mathbf{A}_{k}^{(\mathcal{E}, \psi)}(t, x):=w_{k} \psi(x) \int_{-\infty}^{t} \mathcal{E}_{s} \mathrm{~d} s
$$

Here, $\left\{e_{k}\right\}_{k=1}^{d}$ is the canonical orthonormal basis of $\mathbb{R}^{d}$. By (72), the vector potential $\mathbf{A}^{(\mathcal{E}, \psi)} \in \mathbf{C}_{0}^{\infty}$ satisfies the AC-condition (13) for sufficiently large times $t$. Then, we infer from Theorem 4.1 (p), (65) and (66) applied to the vector potential $\mathbf{A}^{(\mathcal{E}, \psi)}$ that

$$
\frac{1}{2} \int_{\mathbb{R}} \mathrm{d} s_{1} \int_{\mathbb{R}} \mathrm{d} s_{2} \boldsymbol{\sigma}_{\mathrm{p}}\left(s_{1}-s_{2}\right) \mathcal{E}_{s_{2}} \mathcal{E}_{s_{1}} \geq 0
$$

for all $\mathcal{E} \in C_{0}^{\infty}(\mathbb{R})$ satisfying (72). The latter is nothing but the density of heat finally produced within the fermion system when the electromagnetic field $\mathbf{A}^{(\mathcal{E}, \psi)}$ is turned off. 
Since $\boldsymbol{\sigma}_{\mathrm{p}} \equiv \boldsymbol{\sigma}_{\mathrm{p}}^{(\beta, \lambda)}$ is a continuous function obeying $\boldsymbol{\sigma}_{\mathrm{p}}(t)=\boldsymbol{\sigma}_{\mathrm{p}}(-t)$ and $\boldsymbol{\sigma}_{\mathrm{p}}(0)=0$, it is then straightforward to verify that the real numbers $\boldsymbol{\sigma}_{\mathrm{p}}(t), t \in \mathbb{R}$, are unique and only depend on the inverse temperature $\beta \in \mathbb{R}^{+}$and the strength $\lambda \in \mathbb{R}_{0}^{+}$of disorder. Moreover, by (75), the in-phase paramagnetic conductivity $\sigma_{\mathrm{p}}$ is the kernel of a positive quadratic form on the space of smooth, compactly supported functions $\mathcal{E} \in C_{0}^{\infty}(\mathbb{R})$ satisfying (72). We show below that this positivity property of $\sigma_{\mathrm{p}}$ together with the Bochner-Schwartz theorem leads to the existence of the $\mathrm{AC}$-conductivity measure for the system of lattice fermions considered here:

\section{Theorem 4.3 (AC-conductivity measure)}

For any $\beta \in \mathbb{R}^{+}$and $\lambda \in \mathbb{R}_{0}^{+}$, there is a positive measure $\mu_{\mathrm{AC}} \equiv \mu_{\mathrm{AC}}^{(\beta, \lambda)}$ of at most polynomial growth on $\mathbb{R} \backslash\{0\}$ and a constant $D \in \mathbb{R}_{0}^{+}$such that, for any $\mathbf{A} \in \mathbf{C}_{0}^{\infty}$ and $t \geq t_{1}$,

$$
\begin{aligned}
\mathbf{s}(t)=\mathfrak{i}_{\mathrm{p}}(t)= & \frac{1}{2} \int_{\mathbb{R}^{d}} \mathrm{~d}^{d} x \int_{\mathbb{R} \backslash\{0\}} \mu_{\mathrm{AC}}(\mathrm{d} \nu)\left\langle\hat{E}_{\mathbf{A}}(\nu, x), \hat{E}_{\mathbf{A}}(\nu, x)\right\rangle \\
& +\left.D \int_{\mathbb{R}^{d}} \mathrm{~d}^{d} x\left|\partial_{\nu} \hat{E}_{\mathbf{A}}(\nu, x)\right|_{\nu=0}\right|^{2}
\end{aligned}
$$

with $\hat{E}_{\mathbf{A}}$ being the Fourier transform of the electromagnetic field $E_{\mathbf{A}}$ (12).

Proof: $\quad$ Fix $\beta \in \mathbb{R}^{+}$and $\lambda \in \mathbb{R}_{0}^{+}$. It follows from (75) that

$$
\int_{\mathbb{R}} \boldsymbol{\sigma}_{\mathrm{p}}(s)[\tilde{\varphi} * \varphi](s) \mathrm{d} s:=\int_{\mathbb{R}} \mathrm{d} s_{1} \boldsymbol{\sigma}_{\mathrm{p}}\left(s_{1}\right) \int_{\mathbb{R}} \mathrm{d} s_{2} \varphi\left(s_{2}-s_{1}\right) \varphi\left(s_{2}\right) \geq 0
$$

for all $\varphi \in C_{0}^{\infty}(\mathbb{R})$ satisfying the first condition of (72). Here, $\tilde{\varphi}$ is the function defined by $\tilde{\varphi}(s):=\varphi(-s)$ for any $s \in \mathbb{R}$. For all $\varphi \in C_{0}^{\infty}(\mathbb{R})$, observe that $\varphi^{\prime} \in C_{0}^{\infty}(\mathbb{R})$ and

$$
\int_{\mathbb{R}} \varphi^{\prime}(s) \mathrm{d} s=0 .
$$

Using this in order to get rid of the first assumption in (72), i.e., the AC-condition, we define the real-valued distribution $\varsigma \equiv \varsigma^{(\beta, \lambda)}$ by

$$
\varsigma(\varphi):=-\int_{\mathbb{R}} \boldsymbol{\sigma}_{\mathrm{p}}(s) \varphi^{\prime \prime}(s) \mathrm{d} s, \quad \varphi \in C_{0}^{\infty}(\mathbb{R}) .
$$

Indeed, by (76)-(77), note that

$$
\varsigma(\tilde{\varphi} * \varphi)=\int_{\mathbb{R}} \boldsymbol{\sigma}_{\mathrm{p}}(s)\left[\widetilde{\left(\varphi^{\prime}\right)} * \varphi^{\prime}\right](s) \mathrm{d} s \geq 0 .
$$


Since $\sigma_{\mathrm{p}}(t)=\sigma_{\mathrm{p}}(-t)$, this equality can easily be extended to complex-valued functions $\varphi \in C_{0}^{\infty}(\mathbb{R} ; \mathbb{C})$ by replacing $\tilde{\varphi}$ with its conjugate $\overline{\tilde{\varphi}}$. So, the distribution $\varsigma$ is of positive type. In particular, applying the Bochner-Schwartz theorem [RS2, Theorem IX.10] we deduce that $\varsigma$ is a tempered distribution which is the Fourier transform of a positive measure $\mu_{\varsigma}$ of at most polynomial growth. Then define the measure $\mu_{\mathrm{AC}} \equiv \mu_{\mathrm{AC}}^{(\beta, \lambda)}$ as the restriction of $\nu^{-2} \mu_{\varsigma}(\mathrm{d} \nu)$ on $\mathbb{R} \backslash\{0\}$ and observe that

$$
\begin{aligned}
\mathbf{s}(t)= & \int_{\mathbb{R}^{d}} \mathrm{~d}^{d} x \int_{t_{0}}^{t} \mathrm{~d} s_{1} \int_{t_{0}}^{s_{1}} \mathrm{~d} s_{2} \boldsymbol{\sigma}_{\mathrm{p}}\left(s_{1}-s_{2}\right)\left\langle E_{\mathbf{A}}\left(s_{1}, x\right), E_{\mathbf{A}}\left(s_{2}, x\right)\right\rangle \\
= & \frac{1}{2} \int_{\mathbb{R}^{d}} \mathrm{~d}^{d} x \int_{\mathbb{R}_{\{0\}}} \mu_{\mathrm{AC}}(\mathrm{d} \nu)\left\langle\hat{E}_{\mathbf{A}}(\nu, x), \hat{E}_{\mathbf{A}}(\nu, x)\right\rangle \\
& +\mu_{\varsigma}(\{0\}) \int_{\mathbb{R}^{d}} \mathrm{~d}^{d} x\left|\int_{\mathbb{R}} \mathrm{d} s \mathbf{A}(s, x)\right|^{2}
\end{aligned}
$$

for any $\mathbf{A} \in \mathbf{C}_{0}^{\infty}$ and $t \geq t_{1}$, using Theorem 4.1, Fubini's theorem, as well as Equations (14) and (67).

This theorem uniquely defines the measure $\mu_{\mathrm{AC}}$, named the (in-phase) $A C$-conductivity measure. It characterizes the heat production per unit volume due to the component of frequency $\nu \in \mathbb{R} \backslash\{0\}$ of the electric field, in accordance with Joule's law in the AC-regime.

In fact, because of the restriction on functions satisfying the AC-condition (72), (76) is weaker than the condition defining functions of positive type. Above we overcome this problem by introducing the distribution $\varsigma$ which is clearly of positive type, but only as a general tempered distribution. In the subsequent paper we will show that the function $\sigma_{\mathrm{p}}$, the in-phase paramagnetic conductivity, is indeed of positive type up to a constant. This allows us to use the original theorem of Bochner [RS2, Theorem IX.9] on functions of positive type (or the spectral theorem) to show the existence of a finite positive measure $\mu_{\mathrm{p}}$ such that

$$
\boldsymbol{\sigma}_{\mathrm{p}}(t)=\int_{\mathbb{R}}(\cos (t \nu)-1) \mu_{\mathrm{p}}(\mathrm{d} \nu), \quad t \in \mathbb{R},
$$

similar to [BPK2, Theorem 3.1]. Observe that this fact implies, in particular, that Theorem 4.3 actually holds with $D=0$. Such analysis is technically a bit more involved than the proof above and requires the use of the analyticity of timecorrelation functions of KMS states, but has the advantage of automatically implying the finiteness of $\mu_{\mathrm{p}}$ with $\mu_{\mathrm{AC}}=\left.\mu_{\mathrm{p}}\right|_{\mathbb{R} \backslash\{0\}}$, similar to the microscopic case [BPK2, Theorem 3.1]. 
Note that the case $\lambda=0$ can be interpreted as the perfect conductor. We show in the subsequent paper that

$$
\mu_{\mathrm{AC}}^{(\beta, 0)}(\mathbb{R} \backslash\{0\})=0
$$

and hence, the heat production vanishes in this special case. Analogously, the limit $\lambda \rightarrow \infty$ corresponds to the perfect insulator and also leads to a vanishing heat production. Positivity of the AC-conductivity measure means that the fermion system cannot transfer any energy to the electromagnetic field. This property is a consequence of the second principle of thermodynamics. In fact, the fermion system even absorbs, in general, some non-vanishing amount of electromagnetic energy. These points will be all addressed in the subsequent paper.

\section{Remark 4.4}

For electric fields slowly varying in time, charge carriers have time to move and significantly change the charge density, producing an additional, self-generated, internal electric field. This contribution is not taken into account in our model. Thus, the physical meaning of the behavior of the AC-conductivity measure $\mu_{\mathrm{AC}}$ at low frequencies is not clear, in general. However, if one imposes regularity of $\mu_{\mathrm{AC}}$ near $\nu=0$ then this behavior becomes physically relevant. One natural way to obtain some regularity of $\mu_{\mathrm{AC}}$ at low frequencies is to avoid the presence of free charge carriers by imposing some localization condition. This is done, for instance, in [KLM] where the validity of Mott's formula for the conductivity of quantum mechanical charged carriers is studied.

\section{Technical Proofs}

We start our study by two technical results that are used in various proofs of Sections 5.3-5.5: a decomposition of complex-time two-point correlation functions (Section 5.1) and a relatively simple extension of the Akcoglu-Krengel ergodic theorem to non-regular sequences (Section 5.2). Then, we tackle the proof of Theorem 4.1 in Sections 5.3-5.4. Finally, Section 5.5 justifies the construction done in Section 3.3. In particular, we prove Theorem 3.5 in that subsection.

\subsection{Complex-Time Two-Point Correlation Functions}

By [BPK2, Lemma 5.2], the microscopic paramagnetic transport coefficient (29) can be expressed in terms of complex-time two-point correlation functions. The 
latter are explicitly given in terms of quantities involving the Anderson tightbinding Hamiltonian (Section 2.1). Indeed, by [BPK2, Eq. (101)], for all $\beta \in \mathbb{R}^{+}$, $\omega \in \Omega, \lambda \in \mathbb{R}_{0}^{+}, t \in \mathbb{R}$, and $\alpha \in[0, \beta]$,

$$
C_{t+i \alpha}^{(\omega)}(\mathbf{x})=\left\langle\mathfrak{e}_{x^{(2)}}, \mathrm{e}^{-i t\left(\Delta_{\mathrm{d}}+\lambda V_{\omega}\right)} F_{\alpha}^{\beta}\left(\Delta_{\mathrm{d}}+\lambda V_{\omega}\right) \mathfrak{e}_{x^{(1)}}\right\rangle, \quad \mathbf{x}:=\left(x^{(1)}, x^{(2)}\right) \in \mathfrak{L}^{2},
$$

where $F_{\alpha}^{\beta}$ is the real function defined, for every $\beta \in \mathbb{R}^{+}$and $\alpha \in \mathbb{R}$, by

$$
F_{\alpha}^{\beta}(\varkappa):=\frac{\mathrm{e}^{\alpha \varkappa}}{1+\mathrm{e}^{\beta \varkappa}}, \quad \varkappa \in \mathbb{R} .
$$

Equation (78) provides useful estimates like space-decay properties of $C_{t+i \alpha}^{(\omega)}$. Note that the notation $\|\cdot\|_{\text {op }}$ stands for the operator norm.

\section{Theorem 5.1 (Decomposition of two-point correlation functions)}

For any $\varepsilon, \beta \in \mathbb{R}^{+}, \omega \in \Omega, \lambda \in \mathbb{R}_{0}^{+}, t \in \mathbb{R}, v \in(0, \beta / 2)$ and $\alpha \in[v, \beta-v]$, the complex-time two-point correlation function $C_{t+i \alpha}^{(\omega)}$ can be decomposed as

$$
C_{t+i \alpha}^{(\omega)}(\mathbf{x})=A_{t+i \alpha, v, \varepsilon}^{(\omega)}(\mathbf{x})+B_{t+i \alpha, v, \varepsilon}^{(\omega)}(\mathbf{x}), \quad \mathbf{x}:=\left(x^{(1)}, x^{(2)}\right) \in \mathfrak{L}^{2},
$$

where $A_{t+i \alpha, v, \varepsilon}^{(\omega)}(\cdot)$ and $B_{t+i \alpha, v, \varepsilon}^{(\omega)}(\cdot)$ are kernels (w.r.t. $\left.\left\{\mathfrak{e}_{x}\right\}_{x \in \mathfrak{L}}\right)$ of bounded operators $A_{t+i \alpha, v, \varepsilon}^{(\omega)} \equiv A_{t+i \alpha, v, \varepsilon}^{(\beta, \omega, \lambda)}$ and $B_{t+i \alpha, v, \varepsilon}^{(\omega)} \equiv B_{t+i \alpha, v, \varepsilon}^{(\beta, \omega, \lambda)}$ acting on $\ell^{2}(\mathfrak{L})$ and satisfying the following properties:

(i) Boundedness: There is a finite constant $D \in \mathbb{R}^{+}$only depending on $\beta, v$ such that

$$
\left\|A_{t+i \alpha, v, \varepsilon}^{(\omega)}\right\|_{\mathrm{op}} \leq \varepsilon \quad \text { and } \quad\left\|B_{t+i \alpha, v, \varepsilon}^{(\omega)}\right\|_{\mathrm{op}} \leq D .
$$

(ii) Decay: If $T \in \mathbb{R}^{+}$and $t \in[-T, T]$, then there is a finite constant $D \in \mathbb{R}^{+}$ only depending on $\varepsilon, \beta, v, d, T$ such that

$$
\left|B_{t+i \alpha, v, \varepsilon}^{(\omega)}(\mathbf{x})\right| \leq \frac{D}{1+\left|x^{(1)}-x^{(2)}\right| d^{2}+1}, \quad \mathbf{x} \in \mathfrak{L}^{2} .
$$

(iii) Continuity w.r.t. times: If $T \in \mathbb{R}^{+}$and $s_{1}, s_{2} \in[-T, T]$, then there is a finite constant $\eta \in \mathbb{R}^{+}$only depending on $\varepsilon, \beta, v, d, T$ such that

$$
\left|B_{s_{1}+i \alpha, v, \varepsilon}^{(\omega)}(\mathbf{x})-B_{s_{2}+i \alpha, v, \varepsilon}^{(\omega)}(\mathbf{x})\right| \leq \frac{\varepsilon(1+\lambda)}{1+\left|x^{(1)}-x^{(2)}\right| d^{2}+1}, \quad \mathbf{x} \in \mathfrak{L}^{2},
$$


whenever $\left|s_{2}-s_{1}\right| \leq \eta$.

(iv) Continuity w.r.t. random variables: For any $\mathrm{x} \in \mathfrak{L}^{2}$, the maps

$$
\omega \mapsto C_{t+i \alpha}^{(\omega)}(\mathbf{x}), \omega \mapsto A_{t+i \alpha, v, \varepsilon}^{(\omega)}(\mathbf{x}), \omega \mapsto B_{t+i \alpha, v, \varepsilon}^{(\omega)}(\mathbf{x})
$$

from $\Omega$ to $\mathbb{R}$ are continuous w.r.t. the topology on $\Omega$ of which $\mathfrak{A}_{\Omega}$ is the Borel $\sigma$-algebra .

Proof: (i) The spectral theorem applied to the bounded self-adjoint operator $\left(\Delta_{\mathrm{d}}+\lambda V_{\omega}\right) \in \mathcal{B}\left(\ell^{2}(\mathfrak{L})\right)$ implies from (78) that

$$
C_{t+i \alpha}^{(\omega)}(\mathbf{x})=\int F_{\alpha}^{\beta}(\varkappa) \mathrm{e}^{-i t \varkappa} \mathrm{d} \kappa_{\mathbf{x}}^{(\omega)}(\varkappa)
$$

with $\kappa_{\mathbf{x}}^{(\omega)} \equiv \kappa_{\mathbf{x}}^{(\omega, \lambda)}$ being the spectral measure of $\left(\Delta_{\mathrm{d}}+\lambda V_{\omega}\right)$ w.r.t. $\mathfrak{e}_{x^{(1)}}, \mathfrak{e}_{x^{(2)}} \in$ $\ell^{2}(\mathfrak{L})$. Note that $F_{\alpha}^{\beta}(79)$ is a Schwartz function for all $\beta \in \mathbb{R}^{+}$and $\alpha \in(0, \beta)$. Therefore, its Fourier transform $\hat{F}_{\alpha}^{\beta}$ is again a Schwartz function. Moreover, for all $\beta>0$ and $v \in(0, \beta / 2)$, there is a finite constant $D_{\beta, v} \in \mathbb{R}^{+}$such that, for any $\alpha \in[v, \beta-v]$ and all $\nu \in \mathbb{R}$,

$$
\left|\hat{F}_{\alpha}^{\beta}(\nu)\right| \leq \frac{D_{\beta, v}}{1+\nu^{2}} .
$$

In particular, for any $\varepsilon \in \mathbb{R}^{+}$, there is $M_{\beta, v, \varepsilon} \in \mathbb{R}^{+}$such that

$$
\int_{|\nu| \geq M_{\beta, v, \varepsilon}}\left|\hat{F}_{\alpha}^{\beta}(\nu)\right| \mathrm{d} \nu \leq \int_{|\nu| \geq M_{\beta, v, \varepsilon}} \frac{D_{\beta, v}}{1+\nu^{2}} \mathrm{~d} \nu<\varepsilon .
$$

For any $\varepsilon, \beta \in \mathbb{R}^{+}, v \in(0, \beta / 2)$ and $\alpha \in[v, \beta-v]$, we then decompose the function $F_{\alpha}^{\beta}$ into two orthogonal functions of $\varkappa \in \mathbb{R}$ :

$$
\begin{aligned}
f_{v, \varepsilon, \alpha}^{\beta}(\varkappa) & :=\int_{|\nu| \geq M_{\beta, v, \varepsilon}} \hat{F}_{\alpha}^{\beta}(\nu) \mathrm{e}^{i \nu \varkappa} \mathrm{d} \nu, \\
g_{v, \varepsilon, \alpha}^{\beta}(\varkappa) & :=\int_{|\nu|<M_{\beta, v, \varepsilon}} \hat{F}_{\alpha}^{\beta}(\nu) \mathrm{e}^{i \nu \varkappa} \mathrm{d} \nu .
\end{aligned}
$$

Now, for any $\varepsilon, \beta \in \mathbb{R}^{+}, \omega \in \Omega, \lambda \in \mathbb{R}_{0}^{+}, t \in \mathbb{R}, v \in(0, \beta / 2)$ and $\alpha \in[v, \beta-v]$, define the bounded operators $A_{t+i \alpha, v, \varepsilon}^{(\omega)} \equiv A_{t+i \alpha, v, \varepsilon}^{(\beta, \omega, \lambda)}$ and $B_{t+i \alpha, v, \varepsilon}^{(\omega)} \equiv B_{t+i \alpha, v, \varepsilon}^{(\beta, \omega, \lambda)}$ acting on $\ell^{2}(\mathfrak{L})$ by their kernels

$$
\begin{aligned}
\left\langle\mathfrak{e}_{x^{(2)}}, A_{t+i \alpha, v, \varepsilon}^{(\omega)} \mathfrak{e}_{x^{(1)}}\right\rangle & \equiv A_{t+i \alpha, v, \varepsilon}^{(\omega)}(\mathbf{x}):=\int f_{v, \varepsilon, \alpha}^{\beta}(\varkappa) \mathrm{e}^{-i t \varkappa} \mathrm{d} \kappa_{\mathbf{x}}^{(\omega)}(\varkappa) \\
\left\langle\mathfrak{e}_{x^{(2)}}, B_{t+i \alpha, v, \varepsilon}^{(\omega)} \mathfrak{e}_{x^{(1)}}\right\rangle & \equiv B_{t+i \alpha, v, \varepsilon}^{(\omega)}(\mathbf{x}):=\int g_{v, \varepsilon, \alpha}^{\beta}(\varkappa) \mathrm{e}^{-i t \varkappa} \mathrm{d} \kappa_{\mathbf{x}}^{(\omega)}(\varkappa)
\end{aligned}
$$


for any $\mathbf{x} \in \mathfrak{L}^{2}$. Indeed, by construction (cf. (81)-(82)),

$$
\left\|A_{t+i \alpha, v, \varepsilon}^{(\omega)}\right\|_{\mathrm{op}} \leq \varepsilon \quad \text { and } \quad\left\|B_{t+i \alpha, v, \varepsilon}^{(\omega)}\right\|_{\mathrm{op}} \leq \pi D_{\beta, v}
$$

for all $\varepsilon, \beta \in \mathbb{R}^{+}, \omega \in \Omega, \lambda \in \mathbb{R}_{0}^{+}, t \in \mathbb{R}, v \in(0, \beta / 2)$ and $\alpha \in[v, \beta-v]$. By (80), recall that $D_{\beta, v}$ only depends on $\beta$ and $v \in(0, \beta / 2)$.

(ii) We first invoke Fubini's theorem to observe from (83)-(85) that

$$
\begin{aligned}
B_{t+i \alpha, v, \varepsilon}^{(\omega)}(\mathbf{x}) & =\int_{|\nu|<M_{\beta, v, \varepsilon}} \mathrm{d} \nu \hat{F}_{\alpha}^{\beta}(\nu) \int \mathrm{d} \kappa_{\mathbf{x}}^{(\omega)}(\varkappa) \mathrm{e}^{-i \varkappa(t-\nu)} \\
& =\int_{|\nu|<M_{\beta, v, \varepsilon}} \mathrm{d} \nu \hat{F}_{\alpha}^{\beta}(\nu)\left\langle\mathfrak{e}_{x^{(2)}}, \mathrm{e}^{-i(t-\nu)\left(\Delta_{\mathrm{d}}+\lambda V_{\omega}\right)} \mathfrak{e}_{x^{(1)}}\right\rangle
\end{aligned}
$$

for all $\mathbf{x} \in \mathfrak{L}^{2}$. If $T \in \mathbb{R}^{+}, t \in[-T, T]$ and $|\nu|<M_{\beta, v, \varepsilon}$, then

$$
(t-\nu) \in\left[-M_{\beta, v, \varepsilon}-T, M_{\beta, v, \varepsilon}+T\right] .
$$

Thus, by [BPK1, Lemma 4.2] with $\epsilon=d^{2}-d+1(d \in \mathbb{N})$, for any $\varepsilon, \beta, T \in \mathbb{R}^{+}$ and $v \in(0, \beta / 2)$, there is a finite constant $\tilde{D}_{\beta, v, \varepsilon, T, d} \in \mathbb{R}^{+}$such that

$$
\left|\left\langle\mathfrak{e}_{x^{(2)}}, \mathrm{e}^{-i(t-\nu)\left(\Delta_{\mathrm{d}}+\lambda V_{\omega}\right)} \mathfrak{e}_{x^{(1)}}\right\rangle\right| \leq \frac{\tilde{D}_{\beta, v, \varepsilon, T, d}}{1+\left|x^{(1)}-x^{(2)}\right|^{d^{2}+1}}
$$

for all $\omega \in \Omega, \lambda \in \mathbb{R}_{0}^{+}, t \in[-T, T], \nu \in\left[-M_{\beta, v, \varepsilon}, M_{\beta, v, \varepsilon}\right]$ and $\mathbf{x} \in \mathfrak{L}^{2}$. We now combine this last inequality with (80) and (86) to derive the bound

$$
\left|B_{t+i \alpha, v, \varepsilon}^{(\omega)}(\mathbf{x})\right| \leq \frac{\pi D_{\beta, v} \tilde{D}_{\beta, v, \varepsilon, T, d}}{1+\left|x^{(1)}-x^{(2)}\right| d^{2}+1}, \quad \mathbf{x} \in \mathfrak{L}^{2} .
$$

(iii) By Equation (86), note that

$$
\partial_{t} B_{t+i \alpha, v, \varepsilon}^{(\omega)}(\mathbf{x})=-i \int_{|\nu|<M_{\beta, v, \varepsilon}} \mathrm{d} \nu \hat{F}_{\alpha}^{\beta}(\nu)\left\langle\left(\Delta_{\mathrm{d}}+\lambda V_{\omega}\right) \mathfrak{e}_{x^{(2)}}, \mathrm{e}^{-i(t-\nu)\left(\Delta_{\mathrm{d}}+\lambda V_{\omega}\right)} \mathfrak{e}_{x^{(1)}}\right\rangle
$$

for all $\varepsilon, \beta \in \mathbb{R}^{+}, \omega \in \Omega, \lambda \in \mathbb{R}_{0}^{+}, t \in \mathbb{R}, v \in(0, \beta / 2), \alpha \in[v, \beta-v]$ and $\mathbf{x} \in \mathfrak{L}^{2}$. Since, for any $\mathbf{x} \in \mathfrak{L}^{2}$,

$$
\begin{aligned}
& \left\langle\left(\Delta_{\mathrm{d}}+\lambda V_{\omega}\right) \mathfrak{e}_{x^{(2)}}, \mathrm{e}^{-i(t-\nu)\left(\Delta_{\mathrm{d}}+\lambda V_{\omega}\right)} \mathfrak{e}_{x^{(1)}}\right\rangle \\
= & -\sum_{z \in \mathfrak{L},|z|=1}\left\langle\mathfrak{e}_{x^{(2)}+z}, \mathrm{e}^{-i(t-\nu)\left(\Delta_{\mathrm{d}}+\lambda V_{\omega}\right)} \mathfrak{e}_{x^{(1)}}\right\rangle \\
& +\left(\lambda V_{\omega}\left(x^{(2)}\right)+2 d\right)\left\langle\mathfrak{e}_{x^{(2)}}, \mathrm{e}^{-i(t-\nu)\left(\Delta_{\mathrm{d}}+\lambda V_{\omega}\right)} \mathfrak{e}_{x^{(1)}}\right\rangle,
\end{aligned}
$$


we use again (80) and (87) together with (88) and $\left|V_{\omega}(x)\right| \leq 1$ to arrive at the third assertion.

(iv) Take any sequence $\left\{\omega_{n}\right\}_{n=1}^{\infty} \subset \Omega$ converging to $\omega_{\infty} \in \Omega$ w.r.t. the topology of which $\mathfrak{A}_{\Omega}$ is the Borel $\sigma$-algebra. This means that the functions $\omega_{n}: \mathfrak{L} \rightarrow[-1,1]$, $n \in \mathbb{N}$, converges pointwise to $\omega_{\infty}$, as $n \rightarrow \infty$. By Lebesgue's dominated convergence theorem, it follows that the sequence $\left\{\Delta_{\mathrm{d}}+\lambda V_{\omega_{n}}\right\}_{n=1}^{\infty}$ of uniformly bounded operators at fixed $\lambda \in \mathbb{R}_{0}^{+}$converges strongly to $\Delta_{\mathrm{d}}+\lambda V_{\omega_{\infty}}$. By [RS1, Chap. VIII, Problem 28 and Theorem VIII.20 (b)], for any bounded and continuous function $\varphi$ on $\mathbb{R}$, the sequence $\left\{\varphi\left(\Delta_{\mathrm{d}}+\lambda V_{\omega_{n}}\right)\right\}_{n=1}^{\infty}$ converges also strongly to $\varphi\left(\Delta_{\mathrm{d}}+\lambda V_{\omega_{\infty}}\right)$.

Now, similar to Equation (78), Definitions (84)-(85) can be rewritten as

$$
\begin{aligned}
& A_{t+i \alpha, v, \varepsilon}^{(\omega)}(\mathbf{x})=\left\langle\mathfrak{e}_{x^{(2)}}, \mathrm{e}^{-i t\left(\Delta_{\mathrm{d}}+\lambda V_{\omega}\right)} f_{v, \varepsilon, \alpha}^{\beta}\left(\Delta_{\mathrm{d}}+\lambda V_{\omega}\right) \mathfrak{e}_{x^{(1)}}\right\rangle \\
& B_{t+i \alpha, v, \varepsilon}^{(\omega)}(\mathbf{x})=\left\langle\mathfrak{e}_{x^{(2)}}, \mathrm{e}^{-i t\left(\Delta_{\mathrm{d}}+\lambda V_{\omega}\right)} g_{v, \varepsilon, \alpha}^{\beta}\left(\Delta_{\mathrm{d}}+\lambda V_{\omega}\right) \mathfrak{e}_{x^{(1)}}\right\rangle
\end{aligned}
$$

for every $\mathbf{x} \in \mathfrak{L}^{2}$. By (79) and (82)-(83), for any $\varepsilon, \beta \in \mathbb{R}^{+}, \omega \in \Omega, \lambda \in \mathbb{R}_{0}^{+}$, $t \in \mathbb{R}, v \in(0, \beta / 2)$ and $\alpha \in[v, \beta-v], F_{\alpha}^{\beta}, f_{v, \varepsilon, \alpha}^{\beta}$ and $g_{v, \varepsilon, \alpha}^{\beta}$ are bounded and continuous function on $\mathbb{R}$. Therefore, for every $\mathbf{x} \in \mathfrak{L}^{2}$ and as $n \rightarrow \infty$, the correlation functions $A_{t+i \alpha, v, \varepsilon}^{\left(\omega_{n}\right)}(\mathbf{x}), B_{t+i \alpha, v, \varepsilon}^{\left(\omega_{n}\right)}(\mathbf{x})$ and $C_{t+i \alpha}^{\left(\omega_{n}\right)}(\mathbf{x})$ converges to $A_{t+i \alpha, v, \varepsilon}^{\left(\omega_{\infty}\right)}(\mathbf{x}), B_{t+i \alpha, v, \varepsilon}^{\left(\omega_{\infty}\right)}(\mathbf{x})$ and $C_{t+i \alpha}^{\left(\omega_{\infty}\right)}(\mathbf{x})$, respectively.

Better estimates on complex-time two-point correlation functions $C_{t+i \alpha}^{(\omega)}$ can certainly be obtained by using that the spectrum of the self-adjoint operator $\left(\Delta_{\mathrm{d}}+\right.$ $\left.\lambda V_{\omega}\right)$ belongs to some ( $\lambda$-dependant) compact set. This property is however not used in Theorem 5.1 to get bounds (i)-(ii) that do not depend on $\lambda \in \mathbb{R}_{0}^{+}$. Note that we only need here the measurability w.r.t. the $\sigma$-algebra $\mathfrak{A}_{\Omega}$ of all operators of Theorem 5.1, which is a direct consequence of their continuity, see Theorem 5.1 (iv).

\subsection{Ergodic Theorem for some Non-Regular Sequences}

The second important ingredient we use in our proofs is the Akcoglu-Krengel ergodic theorem. We present it for completeness. This result is rather standard and can be found in textbooks. Therefore, we keep the exposition as short as possible and only concentrate on results used in this paper. For more details, we recommend [CL]. It is important to note, however, that Theorem 5.5 is a relatively simple extension of [CL, Theorem VI.1.7, Remark VI.1.8] to non-regular sequences. 
We restrict ourselves to additive processes associated with the probability space $\left(\Omega, \mathfrak{A}_{\Omega}, \mathfrak{a}_{\Omega}\right)$ defined in Section 2.1, even if the Akcoglu-Krengel ergodic theorem holds for superadditive or subadditive ones (cf. [CL, Definition VI.1.6]).

\section{Definition 5.2 (Additive process associated with random variables)}

$\left\{\mathfrak{F}^{(\omega)}(\Lambda)\right\}_{\Lambda \in \mathcal{P}_{f}(\mathfrak{L})}$ is an additive process if:

(i) the map $\omega \mapsto \mathfrak{F}^{(\omega)}(\Lambda)$ is bounded and measurable w.r.t. the $\sigma$-algebra $\mathfrak{A}_{\Omega}$ for any $\Lambda \in \mathcal{P}_{f}(\mathfrak{L})$.

(ii) For all disjoint $\Lambda_{1}, \Lambda_{2} \in \mathcal{P}_{f}(\mathfrak{L})$,

$$
\mathfrak{F}^{(\omega)}\left(\Lambda_{1} \cup \Lambda_{2}\right)=\mathfrak{F}^{(\omega)}\left(\Lambda_{1}\right)+\mathfrak{F}^{(\omega)}\left(\Lambda_{2}\right), \quad \omega \in \Omega .
$$

(iii) For all $\Lambda \in \mathcal{P}_{f}(\mathfrak{L})$ and any space shift $x \in \mathfrak{L}$,

$$
\mathbb{E}\left[\mathfrak{F}^{(\omega)}(\Lambda)\right]=\mathbb{E}\left[\mathfrak{F}^{(\omega)}(x+\Lambda)\right] .
$$

The random potentials used here are independently and identically distributed (i.i.d.), see Equation (8), and (91) will trivially hold for the processes we consider below. Recall that $\mathbb{E}[\cdot]$ is the expectation value associated with the probability measure $\mathfrak{a}_{\Omega}$. Note further that additive processes $\left\{\mathfrak{F}^{(\omega)}(\Lambda)\right\}_{\Lambda \in \mathcal{P}_{f}(\mathfrak{L})}$ as defined in Definition 5.2 are superadditive and subadditive in the sense of [CL, Definition VI.1.6].

We now define regular sequences (cf. [CL, Remark VI.1.8]) as follows:

\section{Definition 5.3 (Regular sequences)}

The family $\left\{\Lambda^{(l)}\right\}_{l \in \mathbb{R}^{+}} \subset \mathcal{P}_{f}(\mathfrak{L})$ of non-decreasing (possibly non-cubic) boxes of $\mathfrak{L}$ is a regular sequence if there is a finite constant $D \in \mathbb{R}^{+}$and another non-decreasing sequence of boxes $\left\{\Lambda_{l}\right\}_{l \geq 1}$, given by (25), such that $\mathfrak{L}=\cup_{l \geq 1} \Lambda_{l}$, $\Lambda^{(l)} \subset \Lambda_{l}$ and $0<\left|\Lambda_{l}\right| \leq D\left|\Lambda^{(l)}\right|$ for all $l \geq 1$.

Then, the form of Akcoglu-Krengel ergodic theorem we use in the sequel is the lattice version of [CL, Theorem VI.1.7, Remark VI.1.8] for additive processes associated with the probability space $\left(\Omega, \mathfrak{A}_{\Omega}, \mathfrak{a}_{\Omega}\right)$ :

\section{Theorem 5.4 (Akcoglu-Krengel ergodic theorem)}

Let $\left\{\mathfrak{F}^{(\omega)}(\Lambda)\right\}_{\Lambda \in \mathcal{P}_{f}(\mathfrak{L})}$ be an additive process. Then, for any regular sequence $\left\{\Lambda^{(l)}\right\}_{l \in \mathcal{J}} \subset \mathcal{P}_{f}(\mathfrak{L})$, there is a measurable subset $\tilde{\Omega} \subset \Omega$ of full measure such that, for all $\omega \in \tilde{\Omega}$,

$$
\lim _{\mathcal{J}}\left\{\left|\Lambda^{(l)}\right|^{-1} \mathfrak{F}^{(\omega)}\left(\Lambda^{(l)}\right)\right\}=\mathbb{E}\left[\mathfrak{F}^{(\omega)}(\{0\})\right] .
$$


The Ackoglu-Krengel (superadditive) ergodic theorem, cornerstone of ergodic theory, generalizes the celebrated Birkhoff additive ergodic theorem. Unfortunately, this theorem, in the above form, is not sufficiently general to be applied in our proofs. Indeed, Theorem 5.4 requires regular sequences. This is too restrictive w.r.t. our applications because we have to evaluate space-inhomogeneous limit of the form

$$
\lim _{l \rightarrow \infty} \frac{1}{\left|\Lambda_{l}\right|} \sum_{x \in \Lambda_{l}} \mathfrak{F}^{(\omega)}(\{x\}) f\left(l^{-1} x\right)
$$

with $f \in C_{0}\left(\mathbb{R}^{d}, \mathbb{R}\right)$ and $\left\{\Lambda_{l}\right\}_{l \geq 1}$ defined by (25). See for instance Section 5.3.

To this end, we divide the compact support of $f$, say for simplicity $[-1 / 2,1 / 2]^{d}$, in $n^{d}$ boxes $\left\{b_{j}\right\}_{j \in \mathcal{D}_{n}}$ of side-length $1 / n$, where

$$
\mathcal{D}_{n}:=\{-(n-1) / 2,-(n-3) / 2, \ldots,(n-3) / 2,(n-1) / 2\}^{d} .
$$

Explicitly, for any $j \in \mathcal{D}_{n}$,

$$
b_{j}:=j n^{-1}+n^{-1}[-1 / 2,1 / 2]^{d} \text { and }[-1 / 2,1 / 2]^{d}=\bigcup_{j \in \mathcal{D}_{n}} b_{j} .
$$

We then need to analyze the limit

$$
\lim _{l \rightarrow \infty}\left|\mathfrak{L} \cap\left(l b_{j}\right)\right|^{-1} \mathfrak{F}^{(\omega)}\left(\mathfrak{L} \cap\left(l b_{j}\right)\right)
$$

for $n \in \mathbb{N}$ and $j \in \mathcal{D}_{n}$. However, $\left\{\mathfrak{L} \cap\left(l b_{j}\right)\right\}_{l \in \mathbb{N}}$ is non-regular, in general. For instance, if $n$ is an odd integer then this situation appears for all $j \in \mathcal{D}_{n} \backslash\{(0, \ldots, 0)\}$ because $\left\{\mathfrak{L} \cap\left(l b_{j}\right)\right\}_{l \in \mathbb{N}}$ is not a non-decreasing sequence in this case. To overcome this difficulty, we proof the following extension of Theorem 5.4:

\section{Theorem 5.5 (Ergodic theorem for some non-regular sequences)}

Let $\left\{\mathfrak{F}^{(\omega)}(\Lambda)\right\}_{\Lambda \in \mathcal{P}_{f}(\mathfrak{L})}$ be an additive process. Then, there is a measurable subset $\tilde{\Omega} \subset \Omega$ of full measure such that, for all $\omega \in \tilde{\Omega}, n \in \mathbb{N}$, and $j \in \mathcal{D}_{n}$,

$$
\lim _{l \rightarrow \infty}\left\{\left|\mathfrak{L} \cap\left(l b_{j}\right)\right|^{-1} \mathfrak{F}^{(\omega)}\left(\mathfrak{L} \cap\left(l b_{j}\right)\right)\right\}=\mathbb{E}\left[\mathfrak{F}^{(\omega)}(\{0\})\right] .
$$

Proof: $\quad$ Let $n \in \mathbb{N}$. By Theorem 5.4, we can fix w.l.o.g. the parameter $j \equiv$ $\left(j_{1}, \ldots, j_{d}\right) \in \mathcal{D}_{n}$ such that the family $\left\{\mathfrak{L} \cap\left(l b_{j}\right)\right\}_{l \in \mathbb{N}}$ is non-regular. Then, we take the sequences $\left\{\Lambda^{(l, j)}\right\}_{l \in \mathbb{N}}$ and $\left\{\tilde{\Lambda}^{(l, j)}\right\}_{l \in \mathbb{N}}$ defined, for any $l \in \mathbb{R}^{+}$, by

$$
\Lambda^{(l, j)}:=\left\{\left(x_{1}, \ldots, x_{d}\right) \in \mathfrak{L}: \forall k \in\{1, \ldots, d\},\left|x_{k}\right| \leq l\left(\left|j_{k}\right|+1 / 2\right) n^{-1}+1\right\}
$$


and

$$
\tilde{\Lambda}^{(l, j)}:=\Lambda^{(l, j)} \backslash\left\{\mathfrak{L} \cap\left(l b_{j}\right)\right\} .
$$

In particular,

$$
\mathfrak{F}^{(\omega)}\left(l b_{j}\right)=\mathfrak{F}^{(\omega)}\left(\Lambda^{(l, j)}\right)-\mathfrak{F}^{(\omega)}\left(\tilde{\Lambda}^{(l, j)}\right)
$$

because $\left\{\mathfrak{F}^{(\omega)}(\Lambda)\right\}_{\Lambda \in \mathcal{P}_{f}(\mathfrak{L})}$ is by assumption an additive process. Note that $\left\{\Lambda^{(l, j)}\right\}_{l \in \mathbb{N}}$ is a regular sequence and thus

$$
\lim _{l \rightarrow \infty}\left\{\left|\Lambda^{(l, j)}\right|^{-1} \mathfrak{F}^{(\omega)}\left(\Lambda^{(l, j)}\right)\right\}=\mathbb{E}\left[\mathfrak{F}^{(\omega)}(\{0\})\right]
$$

almost surely, by Theorem 5.4. $\left\{\tilde{\Lambda}^{(l, j)}\right\}_{l \in \mathbb{N}}$ satisfies Definition 5.3, up to the fact that it is not a sequence of boxes. Indeed, we can obtain $\tilde{\Lambda}^{(l, j)}$ by subtracting from $\Lambda^{(l, j)} d$ boxes of the form

$$
\Lambda^{(l, j)} \cap\left\{x \in \mathfrak{L} \mid x_{k} \lessgtr l\left(j_{k} \pm 1 / 2\right) n^{-1} \pm 1\right\}, \quad k=1, \ldots, d,
$$

containing the origin of $\mathfrak{L}$. By applying Theorem 5.4 to the corresponding regular sequences of boxes we arrive at:

$$
\lim _{l \rightarrow \infty}\left\{\left|\tilde{\Lambda}^{(l, j)}\right|^{-1} \mathfrak{F}^{(\omega)}\left(\tilde{\Lambda}^{(l, j)}\right)\right\}=\mathbb{E}\left[\mathfrak{F}^{(\omega)}(\{0\})\right]
$$

We omit the details. Therefore, by Theorem 5.4 and (97), there is a measurable subset $\hat{\Omega}_{j, n} \equiv \hat{\Omega}_{j, n}^{(\beta, \lambda)} \subset \Omega$ of full measure such that, for any $\omega \in \hat{\Omega}_{j, n}$,

$$
\lim _{l \rightarrow \infty}\left\{\left|\mathfrak{L} \cap\left(l b_{j}\right)\right|^{-1} \mathfrak{F}^{(\omega)}\left(\mathfrak{L} \cap\left(l b_{j}\right)\right)\right\}=\mathbb{E}\left[\mathfrak{F}^{(\omega)}(\{0\})\right] .
$$

Note that we have used here that the intersection of any countable intersection of measurable sets of full measure has full measure. This fact is used many times in our proofs.

It follows that (98) holds true for any $n \in \mathbb{N}, j \in \mathcal{D}_{n}$, and $\omega \in \hat{\Omega}_{j, n}$, while the measurable subset defined by

$$
\tilde{\Omega}:=\bigcap_{n \in \mathbb{N}} \bigcap_{j \in \mathcal{D}_{n}} \hat{\Omega}_{j, n} \subset \Omega
$$

has full measure.

Note that the notion of a regular sequence is not completely consistent in the literature. Use used here the definition given in [CL, Remark VI.1.8] and then generalized Theorem 5.4 to some, w.r.t. this definition, non-regular sequences in the above theorem.

Theorem 5.5 directly yields the limit (92): 


\section{Theorem 5.6 (Space-inhomogeneous ergodic theorem)}

Let $\left\{\mathfrak{F}^{(\omega)}(\Lambda)\right\}_{\Lambda \in \mathcal{P}_{f}(\mathfrak{L})}$ be an additive process. Then, for any $f \in C_{0}\left(\mathbb{R}^{d}, \mathbb{R}\right)$, there is a measurable subset $\tilde{\Omega} \subset \Omega$ of full measure such that, for all $\omega \in \tilde{\Omega}$,

$$
\lim _{l \rightarrow \infty} \frac{1}{\left|\Lambda_{l}\right|} \sum_{x \in \Lambda_{l}} \mathfrak{F}^{(\omega)}(\{x\}) f\left(l^{-1} x\right)=\mathbb{E}\left[\mathfrak{F}^{(\omega)}(\{0\})\right] \int_{\mathbb{R}^{d}} f(x) \mathrm{d}^{d} x .
$$

Proof: $\quad$ Since $f \in C_{0}\left(\mathbb{R}^{d}, \mathbb{R}\right)$ has compact support, $f$ is uniformly continuous. Assume w.l.o.g. that

$$
\operatorname{supp}(f) \subset[-1 / 2,1 / 2]^{d} .
$$

Then, there is a finite constant $D$ not depending on $j \in \mathcal{D}_{n}, t \in \mathbb{R}, k \in\{1, \ldots, d\}$ and $x, y \in b_{j}$ such that

$$
|f(x)-f(y)| \leq D n^{-1} .
$$

Using this and Theorem 5.5 we obtain the assertion.

This last theorem could be extended to continuous functions $f \in C\left(\mathbb{R}^{d}, \mathbb{R}\right)$ vanishing sufficiently fast when $|l| \rightarrow \infty$ as well as for ergodic probability measures $\mathfrak{a}_{\Omega}$. This generalization is however not necessary here and we refrain from proving it in detail.

\subsection{Diamagnetic Transport Coefficient and Density}

The aim of this section is to obtain the deterministic diamagnetic transport coefficient $\Xi_{\mathrm{d}}$ as well as the diamagnetic energy density $\mathfrak{i}_{\mathrm{d}}$. See (34) and (60) for their definitions. It is an simple application of the ergodic theorems of Section 5.2 and serves as a sort of "warm up" for the technically more involved case of paramagnetic quantities.

We consider here the limit $l \rightarrow \infty$ of the current density (35) at equilibrium and the space-averaged diamagnetic energy production coefficient $\Xi_{\mathrm{d}, l}^{(\omega)}$ that is defined by (32). Indeed, as explained in Section 3.2, there exist, in general, currents coming from the inhomogeneity of the fermion system for $\lambda \in \mathbb{R}^{+}$, even in absence of electromagnetic fields. We want to prove that, for large samples, there are almost surely no currents within the fermion system at thermal equilibrium. This result yields Assertion (th) of Theorem 3.4. We also would like to show that, as $l \rightarrow \infty, \Xi_{\mathrm{d}, l}^{(\omega)}$ converges almost surely to the diamagnetic transport coefficient $\Xi_{\mathrm{d}}$, see Theorem $3.1(\mathrm{~d})$. 


\section{Corollary 5.7 (Currents and diamagnetic conductivity)}

Let $\beta \in \mathbb{R}^{+}$and $\lambda \in \mathbb{R}_{0}^{+}$. Then one has:

(th) Current densities at thermal equilibrium: For any $z \in \mathbb{Z}^{d}$, there is a measurable subset $\tilde{\Omega}(z) \equiv \tilde{\Omega}^{(\beta, \lambda)}(z) \subset \Omega$ of full measure such that, for all $\omega \in \tilde{\Omega}(z)$,

$$
\lim _{l \rightarrow \infty} \frac{1}{\left|\Lambda_{l}\right|} \sum_{x \in \Lambda_{l}} \varrho^{(\beta, \omega, \lambda)}\left(I_{(x+z, x)}\right)=0 .
$$

(d) Diamagnetic charge transport coefficient: There is a measurable subset $\tilde{\Omega} \equiv$ $\tilde{\Omega}^{(\beta, \lambda)} \subset \Omega$ of full measure such that, for any $\omega \in \tilde{\Omega}$,

$$
\Xi_{\mathrm{d}}:=\lim _{l \rightarrow \infty} \mathbb{E}\left[\Xi_{\mathrm{d}, l}^{(\omega)}\right]=\lim _{l \rightarrow \infty} \Xi_{\mathrm{d}, l}^{(\omega)} \in[-2,2] .
$$

Proof: $\quad$ Let $\beta \in \mathbb{R}^{+}, \lambda \in \mathbb{R}_{0}^{+}$and $z \in \mathbb{Z}^{d}$. We define an additive process $\left\{\mathfrak{F}_{z}^{(\omega)}(\Lambda)\right\}_{\Lambda \in \mathcal{P}_{f}(\mathfrak{L})}$ by

$$
\mathfrak{F}_{z}^{(\omega)}(\Lambda):=\sum_{x \in \Lambda} \varrho^{(\beta, \omega, \lambda)}\left(a_{x+z}^{*} a_{x}\right)=\sum_{x \in \Lambda}\left\langle\mathfrak{e}_{x}, F_{0}^{\beta}\left(\Delta_{\mathrm{d}}+\lambda V_{\omega}\right) \mathfrak{e}_{x+z}\right\rangle
$$

for any finite subset $\Lambda \in \mathcal{P}_{f}(\mathfrak{L})$, see (79) and Definition 5.2. Similar to Theorem 5.1 (iv), the map $\omega \mapsto \mathfrak{F}_{z}^{(\omega)}(\Lambda)$ is bounded and measurable w.r.t. the $\sigma$-algebra $\mathfrak{A}_{\Omega}$ for all $\Lambda \in \mathcal{P}_{f}(\mathfrak{L})$. By the uniqueness of the KMS states $\varrho^{(\beta, \omega, \lambda)}$, we moreover have

$$
\mathfrak{F}_{z}^{(\omega)}\left(z^{\prime}+\Lambda\right)=\mathfrak{F}_{z}^{(\omega)}(\Lambda)
$$

for all $z^{\prime} \in \mathbb{Z}^{d}, \Lambda \in \mathcal{P}_{f}(\mathfrak{L})$ and $\omega \in \Omega$. Clearly, $\left\{\Lambda_{l}\right\}_{l \in \mathbb{R}^{+}} \subset \mathcal{P}_{f}(\mathfrak{L})$ is a regular sequence, see Definition 5.3. Therefore, Theorem 5.4 implies the existence of a measurable subset $\hat{\Omega}(z) \equiv \hat{\Omega}^{(\beta, \lambda)}(z) \subset \Omega$ of full measure such that, for all $\omega \in \tilde{\Omega}(z)$,

$$
\lim _{l \rightarrow \infty}\left\{\left|\Lambda_{l}\right|^{-1} \mathfrak{F}_{z}^{(\omega)}\left(\Lambda_{l}\right)\right\}=\mathbb{E}\left[\varrho^{(\beta, \omega, \lambda)}\left(a_{z}^{*} a_{0}\right)\right]=\mathbb{E}\left[\left\langle\mathfrak{e}_{0}, \mathbf{d}_{\text {fermi }}^{(\beta, \omega, \lambda)} \mathfrak{e}_{z}\right\rangle\right] .
$$

Recall that $\mathbb{E}[\cdot]$ is the expectation value associated with the probability measure $\mathfrak{a}_{\Omega}(8), \mathbf{d}_{\text {fermi }}^{(\beta, \omega, \lambda)}$ is the symbol (23), and $\left\{\mathfrak{e}_{x}\right\}_{x \in \mathfrak{L}}$ is the canonical orthonormal basis of $\ell^{2}(\mathfrak{L})$ with scalar product $\langle\cdot, \cdot\rangle$.

By (26), observe that

$$
\frac{1}{\left|\Lambda_{l}\right|} \sum_{x \in \Lambda_{l}} \varrho^{(\beta, \omega, \lambda)}\left(I_{(x+z, x)}\right)=2 \operatorname{Im}\left\{\left|\Lambda_{l}\right|^{-1} \mathfrak{F}_{z}^{(\omega)}\left(\Lambda_{l}\right)\right\},
$$


while, from the definition (32),

$$
\left\{\Xi_{\mathrm{d}, l}^{(\omega)}\right\}_{k, q}=2 \delta_{k, q} \operatorname{Re}\left\{\left|\Lambda_{l}\right|^{-1} \mathfrak{F}_{e_{k}}^{(\omega)}\left(\Lambda_{l}\right)\right\}
$$

for any $k, q \in\{1, \ldots, d\}$. Combined with (68), (70) and (101), these two equalities yield Assertions (th) and (d), respectively. Indeed, $V_{\omega}$ is an i.i.d. potential and $\mathbb{E}\left[I_{(z, 0)}\right]=0$ for any $z \in \mathfrak{L}$.

We study now the limit $\left(\eta, l^{-1}\right) \rightarrow(0,0)$ of the diamagnetic energy $\mathfrak{I}_{\mathrm{d}}^{\left(\omega, \eta \mathbf{A}_{l}\right)}$ defined by (54). An asymptotic expansion of the diamagnetic energy is given by [BPK2, Theorem 5.12] for small parameters $|\eta| \ll 1$ : For any $\mathbf{A} \in \mathbf{C}_{0}^{\infty}$, there is $\eta_{0} \in \mathbb{R}^{+}$such that, for all $|\eta| \in\left(0, \eta_{0}\right], l, \beta \in \mathbb{R}^{+}, \omega \in \Omega, \lambda \in \mathbb{R}_{0}^{+}$and $t \geq t_{0}$,

$$
\begin{aligned}
\mathfrak{I}_{\mathrm{d}}^{\left(\omega, \eta \mathbf{A}_{l}\right)}(t)= & \frac{\eta}{2} \sum_{\mathbf{x} \in \mathfrak{K}} \varrho^{(\beta, \omega, \lambda)}\left(I_{\mathbf{x}}\right) \int_{t_{0}}^{t} \mathbf{E}_{s}^{\mathbf{A}_{l}}(\mathbf{x}) \mathrm{d} s \\
& +\frac{\eta^{2}}{2} \int_{t_{0}}^{t} \mathrm{~d} s_{1} \int_{t_{0}}^{s_{1}} \mathrm{~d} s_{2} \sum_{\mathbf{x} \in \mathfrak{K}} \sigma_{\mathrm{d}}^{(\omega)}(\mathbf{x}) \mathbf{E}_{s_{2}}^{\mathbf{A}_{l}}(\mathbf{x}) \mathbf{E}_{s_{1}}^{\mathbf{A}_{l}}(\mathbf{x})+\mathcal{O}\left(\eta^{3} l^{d}\right)
\end{aligned}
$$

The correction terms of order $\mathcal{O}\left(\eta^{3} l^{d}\right)$ is uniformly bounded in $\beta \in \mathbb{R}^{+}, \omega \in \Omega$, $\lambda \in \mathbb{R}_{0}^{+}$and $t \geq t_{0}$. Here,

$$
\mathbf{E}_{t}^{\mathbf{A}}(\mathbf{x}) \equiv \mathbf{E}_{t}^{\mathbf{A}}\left(x^{(1)}, x^{(2)}\right):=\int_{0}^{1}\left[E_{\mathbf{A}}\left(t, \alpha x^{(2)}+(1-\alpha) x^{(1)}\right)\right]\left(x^{(2)}-x^{(1)}\right) \mathrm{d} \alpha,
$$

is the integrated electric field between $x^{(2)} \in \mathfrak{L}$ and $x^{(1)} \in \mathfrak{L}$ at time $t \in \mathbb{R}$ and

$$
\mathfrak{K}:=\left\{\mathbf{x}:=\left(x^{(1)}, x^{(2)}\right) \in \mathfrak{L}^{2}:\left|x^{(1)}-x^{(2)}\right|=1\right\}
$$

is the set of bonds of nearest neighbors.

The asymptotic expansion (102) ensures the existence of the limit

$$
\lim _{\eta \rightarrow 0}\left\{\left(\eta\left|\Lambda_{l}\right|\right)^{-1} \mathfrak{I}_{\mathrm{d}}^{\left(\omega, \eta \mathbf{A}_{l}\right)}(t)\right\}=\mathfrak{G}_{l}^{(\omega)}(t)
$$

Here, for any $l, \beta \in \mathbb{R}^{+}, \omega \in \Omega, \lambda \in \mathbb{R}_{0}^{+}, \mathbf{A} \in \mathbf{C}_{0}^{\infty}$ and $t \in \mathbb{R}$, the function

$$
\mathfrak{G}_{l}^{(\omega)}(t) \equiv \mathfrak{G}_{l}^{\left(\beta, \omega, \lambda, \eta \mathbf{A}_{l}\right)}(t)=\frac{1}{2} \int_{t_{0}}^{t} \frac{1}{\left|\Lambda_{l}\right|} \sum_{\mathbf{x} \in \mathfrak{K}} \varrho^{(\beta, \omega, \lambda)}\left(I_{\mathbf{x}}\right) \mathbf{E}_{s}^{\mathbf{A}_{l}}(\mathbf{x}) \mathrm{d} s
$$

is the electric work density produced by thermal currents within the box $\Lambda_{l}$. 
The limit $l \rightarrow \infty$ of the function $\mathfrak{G}_{l}^{(\omega)}$ is a little bit more complicated than in the first two examples because the electric field $\mathbf{E}_{t}^{\mathbf{A}_{l}}$ is space-inhomogeneous. In fact, we can divide the (compact) $\operatorname{support} \operatorname{supp}(\mathbf{A}(t,).) \subset \mathbb{R}^{d}$ of the vector potential $\mathbf{A}(t,$.$) at t \in \mathbb{R}$ in small regions to combine the piecewise-constant approximation of the smooth electric field $E_{\mathbf{A}}$ (12) in (106) with Theorem 5.5. A similar problem is already treated in Theorem 5.6. In fact, one gets the following assertion:

Theorem 5.8 (Electric work density produced by thermal currents)

Let $\beta \in \mathbb{R}^{+}, \lambda \in \mathbb{R}_{0}^{+}$. Then, there is a measurable subset $\tilde{\Omega} \equiv \tilde{\Omega}^{(\beta, \lambda)} \subset \Omega$ of full measure such that, for all $\mathbf{A} \in \mathbf{C}_{0}^{\infty}$,

$$
\lim _{l \rightarrow \infty} \mathfrak{G}_{l}^{(\omega)}(t)=0, \quad \omega \in \tilde{\Omega},
$$

uniformly for all $t \geq t_{0}$.

Proof: We study the limit $l \rightarrow \infty$ of the function

$$
\mathbf{M}_{l}^{(\omega)}(t):=\frac{1}{\left|\Lambda_{l}\right|} \sum_{\mathbf{x} \in \mathfrak{K}} \varrho^{(\beta, \omega, \lambda)}\left(I_{\mathbf{x}}\right) \mathbf{E}_{t}^{\mathbf{A}_{l}}(\mathbf{x})
$$

for any $l, \beta \in \mathbb{R}^{+}, \omega \in \Omega, \lambda \in \mathbb{R}_{0}^{+}, \mathbf{A} \in \mathbf{C}_{0}^{\infty}$ and $t \in \mathbb{R}$. Indeed, because $\mathbf{A} \in \mathbf{C}_{0}^{\infty}$, note that

$$
\left\|\mathbf{E}^{\mathbf{A}}\right\|_{\infty}:=\max \left\{\left|E_{\mathbf{A}}(t, x)\right|:(t, x) \in \operatorname{supp}(A)\right\} \in \mathbb{R}^{+},
$$

which implies that

$$
\sup _{t \in \mathbb{R}}\left|\mathbf{M}_{l}^{(\omega)}(t)\right| \leq\left\|\mathbf{E}^{\mathbf{A}}\right\|_{\infty} \sum_{z \in \mathfrak{L},|z|=1}\left\|I_{(0, z)}\right\|<\infty .
$$

Therefore, by (106) and Lebesgue's dominated convergence theorem, in order to get the assertion it suffices to show that, for any fixed $t \in\left[t_{0}, t_{1}\right]$ and $\omega$ in a subset $\tilde{\Omega} \equiv \tilde{\Omega}^{(\beta, \lambda)} \subset \Omega$ of full measure, the 1.h.s. of (109) vanishes when $l \rightarrow \infty$. This is done like in Theorem 5.6.

Indeed, assume w.l.o.g. that, for all $t \in \mathbb{R}$,

$$
\operatorname{supp}(\mathbf{A}(t, .)) \subset[-1 / 2,1 / 2]^{d} .
$$

For any integer $n \in \mathbb{N}$, we divide the elementary box $[-1 / 2,1 / 2]^{d}$ in $n^{d}$ boxes $\left\{b_{j}\right\}_{j \in \mathcal{D}_{n}}$ of side-length $1 / n$, see (93)-(94). For any $j \in \mathcal{D}_{n}$, let $z^{(j)} \in b_{j}$ be any 
fixed point of the box $b_{j}$. Then, we consider piecewise-constant approximations of the (smooth) electric field (12), that is,

$$
E_{\mathbf{A}}(t, x):=-\partial_{t} \mathbf{A}(t, x), \quad t \in \mathbb{R}, x \in \mathbb{R}^{d},
$$

and define the approximated energy density

$$
\tilde{\mathbf{M}}_{l}^{(\omega)}(t):=\frac{1}{\left|\Lambda_{l}\right|} \sum_{j \in \mathcal{D}_{n}} \sum_{\mathbf{x} \in \mathfrak{K} \cap\left(l b_{j}\right)^{2}} \varrho^{(\beta, \omega, \lambda)}\left(I_{\mathbf{x}}\right)\left[E_{\mathbf{A}}\left(t, z^{(j)}\right)\right]\left(x^{(1)}-x^{(2)}\right)
$$

for any $l, \beta \in \mathbb{R}^{+}, \omega \in \Omega, \lambda \in \mathbb{R}_{0}^{+}, \mathbf{A} \in \mathbf{C}_{0}^{\infty}, t \in \mathbb{R}$ and $n \in \mathbb{N}$.

We infer from (17), (103) and (111) that, for any $l \in \mathbb{R}^{+}, \mathbf{A} \in \mathbf{C}_{0}^{\infty}, j \in \mathcal{D}_{n}$, $t \in \mathbb{R}, k \in\{1, \ldots, d\}$ and $x \in l b_{j}$,

$$
\begin{aligned}
& \left|\mathbf{E}_{t}^{\mathbf{A}_{l}}\left(x, x \pm e_{k}\right)-\left[E_{\mathbf{A}}\left(t, z^{(j)}\right)\right]\left( \pm e_{k}\right)\right| \\
\leq & \int_{0}^{1}\left|\left[\partial_{t} \mathbf{A}\left(t, z^{(j)}\right)\right]\left(e_{k}\right)-\left[\partial_{t} \mathbf{A}_{l}\left(t, x \pm(1-\alpha) e_{k}\right)\right]\left(e_{k}\right)\right| \mathrm{d} \alpha \\
\leq & \sup _{y \in \tilde{b}_{j, l}}\left|\left[\partial_{t} \mathbf{A}\left(t, z^{(j)}\right)\right]\left(e_{k}\right)-\left[\partial_{t} \mathbf{A}(t, y)\right]\left(e_{k}\right)\right|<\infty,
\end{aligned}
$$

where

$$
\tilde{b}_{j, l}:=\left\{x \in \mathbb{R}^{d}: \min _{y \in b_{j}}|x-y| \leq l^{-1}\right\} .
$$

In particular, since $\mathbf{A} \in \mathbf{C}_{0}^{\infty}$, there is a finite constant $D \in \mathbb{R}^{+}$not depending on $j \in \mathcal{D}_{n}, t \in \mathbb{R}, k \in\{1, \ldots, d\}$ and $x \in b_{j}$ such that

$$
\left|\mathbf{E}_{t}^{\mathbf{A}_{l}}\left(x, x \pm e_{k}\right)-\left[E_{\mathbf{A}}\left(t, z^{(j)}\right)\right]\left( \pm e_{k}\right)\right| \leq D\left(n^{-1}+l^{-1}\right) .
$$

This upper bound is the analogue of (99) in the proof of Theorem 5.6. Using also (110), it follows that

$$
\left|\mathbf{M}_{l}^{(\omega)}(t)-\tilde{\mathbf{M}}_{l}^{(\omega)}(t)\right| \leq D\left(n^{-1}+l^{-1}\right) \sum_{z \in \mathfrak{L},|z|=1}\left\|I_{(z, 0)}\right\| .
$$

Therefore, by (104) and (112), for any $z \in \mathbb{Z}^{d}$ such that $|z|=1$, it suffices to compute the limit

$$
\lim _{l \rightarrow \infty} \frac{1}{\left|\mathfrak{L} \cap\left(l b_{j}\right)\right|} \sum_{x \in \mathfrak{L} \cap\left(l b_{j}\right)} \varrho^{(\beta, \omega, \lambda)}\left(I_{(x+z, x)}\right)
$$


like in Theorem 5.6. For any $\beta \in \mathbb{R}^{+}, \lambda \in \mathbb{R}_{0}^{+}$and $z \in \mathbb{Z}^{d}$, we invoke Theorem 5.5 to get the existence of a measurable subset $\hat{\Omega}_{z} \equiv \hat{\Omega}_{z}^{(\beta, \lambda)} \subset \Omega$ of full measure such that, for any $\omega \in \hat{\Omega}_{z}$,

$$
\lim _{l \rightarrow \infty}\left\{\frac{1}{\left|\mathfrak{L} \cap\left(l b_{j}\right)\right|} \sum_{x \in \mathfrak{L} \cap\left(l b_{j}\right)} \varrho^{(\beta, \omega, \lambda)}\left(I_{(x+z, x)}\right)\right\}=\mathbb{E}\left[I_{(z, 0)}\right]=0 .
$$

Note that the last equality is a consequence of the identity $I_{(z, 0)}=-I_{(0, z)}$ and the translation and reflection invariance of the probability measure $\mathfrak{a}_{\Omega}$. Meanwhile, the measurable subset defined by

$$
\tilde{\Omega} \equiv \tilde{\Omega}^{(\beta, \lambda)}:=\bigcap_{z \in \mathfrak{L},|z|=1} \hat{\Omega}_{z} \subset \Omega
$$

has full measure and we obtain from (114)-(115) that, for any $\omega \in \tilde{\Omega}$,

$$
\lim _{l \rightarrow \infty} \sup _{t \in \mathbb{R}}\left|\mathbf{M}_{l}^{(\omega)}(t)\right|=0 \text {. }
$$

It remains to study the diamagnetic energy density $\mathfrak{i}_{\mathrm{d}}$ defined by $(60)$, that is,

$$
\mathfrak{i}_{\mathrm{d}}(t):=\lim _{\eta \rightarrow 0} \lim _{l \rightarrow \infty}\left\{\left(\eta^{2}\left|\Lambda_{l}\right|\right)^{-1} \mathfrak{I}_{\mathrm{d}}^{\left(\omega, \eta \mathbf{A}_{l}\right)}(t)\right\}
$$

for $\beta \in \mathbb{R}^{+}, \omega \in \Omega, \lambda \in \mathbb{R}_{0}^{+}, \mathbf{A} \in \mathbf{C}_{0}^{\infty}$ and $t \geq t_{0}$. Thanks to the asymptotic expansion (102), its derivation is done like in the proof of Theorem 5.8 by replacing current observables $I_{\mathbf{x}}(26)$ and the integrated electric field $\mathbf{E}_{t}^{\mathbf{A}_{l}}(\mathbf{x})$ in Equation (107) with fermion fields $P_{\mathbf{x}}(28)$ and products $\mathbf{E}_{s_{2}}^{\mathbf{A}_{l}}(\mathbf{x}) \mathbf{E}_{s_{1}}^{\mathbf{A}_{l}}(\mathbf{x})$. Then, one gets the diamagnetic energy density $\mathfrak{i}_{\mathrm{d}}$ as stated in Theorem $4.1(\mathrm{~d})$.

\section{Theorem 5.9 (Diamagnetic energy density)}

Let $\beta \in \mathbb{R}^{+}$and $\lambda \in \mathbb{R}_{0}^{+}$. Then, there is a measurable subset $\tilde{\Omega} \equiv \tilde{\Omega}^{(\beta, \lambda)} \subset \Omega$ of full measure such that, for any $\omega \in \tilde{\Omega}$ and $\mathbf{A} \in \mathbf{C}_{0}^{\infty}$,

$$
\mathfrak{i}_{\mathrm{d}}(t)=\int_{\mathbb{R}^{d}} \mathrm{~d}^{d} x \int_{t_{0}}^{t} \mathrm{~d} s_{1} \int_{t_{0}}^{s_{1}} \mathrm{~d} s_{2}\left\langle E_{\mathbf{A}}\left(s_{1}, x\right), \boldsymbol{\Xi}_{\mathrm{d}} E_{\mathbf{A}}\left(s_{2}, x\right)\right\rangle
$$

uniformly for all $t \geq t_{0}$ in compact sets. Here, $\boldsymbol{\Xi}_{\mathrm{d}}$ is defined by (34). 
Proof: $\quad$ Let $\beta \in \mathbb{R}^{+}$and $\lambda \in \mathbb{R}_{0}^{+}$. By (30) and (102), for any $\mathbf{A} \in \mathbf{C}_{0}^{\infty}$, there is $\eta_{0} \in \mathbb{R}^{+}$such that, for all $|\eta| \in\left(0, \eta_{0}\right], l, \beta \in \mathbb{R}^{+}, \omega \in \Omega, \lambda \in \mathbb{R}_{0}^{+}$and $t \geq t_{0}$,

$$
\mathfrak{I}_{\mathrm{d}}^{(\omega, \mathbf{A})}(t)-\eta\left|\Lambda_{l}\right| \mathfrak{G}_{l}^{(\omega)}(t)=\eta^{2}\left|\Lambda_{l}\right| \int_{t_{0}}^{t} \mathrm{~d} s_{1} \int_{t_{0}}^{s_{1}} \mathrm{~d} s_{2} \tilde{\mathbf{X}}_{l}^{(\omega)}\left(s_{1}, s_{2}\right)+\mathcal{O}\left(\eta^{3} l^{d}\right)
$$

with the energy density $\mathfrak{G}_{l}^{(\omega)}$ defined by (106) while

$$
\tilde{\mathbf{X}}_{l}^{(\omega)}\left(s_{1}, s_{2}\right):=\frac{1}{2\left|\Lambda_{l}\right|} \sum_{\mathbf{x} \in \mathfrak{K}} \varrho^{(\beta, \omega, \lambda)}\left(P_{\mathbf{x}}\right) \mathbf{E}_{s_{1}}^{\mathbf{A}_{l}}(\mathbf{x}) \mathbf{E}_{s_{2}}^{\mathbf{A}_{l}}(\mathbf{x})
$$

for any $s_{1}, s_{2} \in \mathbb{R}$. The correction term of order $\mathcal{O}\left(\eta^{3} l^{d}\right)$ is uniformly bounded in $\beta \in \mathbb{R}^{+}, \omega \in \Omega, \lambda \in \mathbb{R}_{0}^{+}$and $t \geq t_{0}$.

Similar to the proof of Theorem 5.8 we use piecewise-constant approximations of the (smooth) electric field and Theorem 5.5 together with (108), (113) and Lebesgue's dominated convergence theorem to compute the limit $l \rightarrow \infty$ of the r.h.s. of (116) (without the factor $\eta^{2} l^{d}$ ). More precisely, one finds the existence a measurable subset $\tilde{\Omega} \equiv \tilde{\Omega}^{(\beta, \lambda)} \subset \Omega$ of full measure such that, for all $\omega \in \tilde{\Omega}$,

$$
\begin{aligned}
& \lim _{l \rightarrow \infty}\left\{\int_{t_{0}}^{t} \mathrm{~d} s_{1} \int_{t_{0}}^{s_{1}} \mathrm{~d} s_{2} \tilde{\mathbf{X}}_{l}^{(\omega)}\left(s_{1}, s_{2}\right)\right\} \\
= & \int_{t_{0}}^{t} \mathrm{~d} s_{1} \int_{t_{0}}^{s_{1}} \mathrm{~d} s_{2} \int_{\mathbb{R}^{d}} \mathrm{~d}^{d} x\left\langle E_{\mathbf{A}}\left(s_{1}, x\right), \boldsymbol{\Xi}_{\mathrm{d}} E_{\mathbf{A}}\left(s_{2}, x\right)\right\rangle
\end{aligned}
$$

uniformly for all $t \geq t_{0}$. The assertion follows from (116) and (118) together with Theorem 5.8 and Fubini's theorem.

\subsection{Paramagnetic Energy Density}

The aim of this section is to prove the existence of the paramagnetic energy density $\mathfrak{i}_{\mathrm{p}}$ defined by (59), that is,

$$
\mathfrak{i}_{\mathrm{p}}(t)=\lim _{\left(\eta, l^{-1}\right) \rightarrow(0,0)}\left\{\left(\eta^{2}\left|\Lambda_{l}\right|\right)^{-1} \mathfrak{I}_{\mathrm{p}}^{\left(\omega, \eta \mathbf{A}_{l}\right)}(t)\right\}
$$

for $\beta \in \mathbb{R}^{+}, \omega \in \Omega, \lambda \in \mathbb{R}_{0}^{+}, \mathbf{A} \in \mathbf{C}_{0}^{\infty}$ and $t \geq t_{0}$. Our proof requires similar arguments to those proving Theorems 5.8-5.9:

- The asymptotic expansion given by [BPK2, Theorem 5.12] for the paramagnetic energy increment. 
- We divide the (compact) support $\operatorname{supp}(\mathbf{A}(t,).) \subset \mathbb{R}^{d}$ of the vector potential $\mathbf{A}(t,$.$) at t \in \mathbb{R}$ in small regions to use the piecewise-constant approximation of the smooth electric field $E_{\mathbf{A}}$.

- Theorem 5.5 and the fact that any countable intersection of measurable sets of full measure has full measure.

- Lebesgue's dominated convergence theorem.

The proof for the paramagnetic case is, however, technically more involved than those of Section 5.3. Indeed, to use [BPK2, Lemma 5.2, Theorem 5.12], we additionally need some (space) decay of complex-time two-point correlation functions. To this end, we invoke Theorem 5.1. The application of the latter requires some technical preparation and we present the corresponding additional arguments in various lemmata which then yield a proposition and a few corollaries and theorems. This rather technical study ends with Theorem 5.21, which serves as a springboard to obtain Theorem 4.1.

First, by [BPK2, Lemma 5.2, Theorem 5.12], for any $\mathbf{A} \in \mathbf{C}_{0}^{\infty}$, there is $\eta_{0} \in$ $\mathbb{R}^{+}$such that, for all $|\eta| \in\left(0, \eta_{0}\right], l, \beta \in \mathbb{R}^{+}, \omega \in \Omega, \lambda \in \mathbb{R}_{0}^{+}$and $t \geq t_{0}$,

$$
\mathfrak{I}_{\mathrm{p}}^{\left(\omega, \eta \mathbf{A}_{l}\right)}(t)=\eta^{2}\left|\Lambda_{l}\right| \int_{t_{0}}^{t} \mathrm{~d} s_{1} \int_{t_{0}}^{s_{1}} \mathrm{~d} s_{2} \mathbf{X}_{l, 0}^{(\omega)}\left(s_{1}, s_{2}\right)+\mathcal{O}\left(\eta^{3} l^{d}\right) .
$$

The correction term of order $\mathcal{O}\left(\eta^{3} l^{d}\right)$ is uniformly bounded in $\beta \in \mathbb{R}^{+}, \omega \in \Omega$, $\lambda \in \mathbb{R}_{0}^{+}$and $t \geq t_{0}$. Here, $\mathbf{X}_{l, v}^{(\omega)}$ is defined, for any $v \in[0, \beta / 2)$ and $s_{1}, s_{2} \in \mathbb{R}$, by

$$
\begin{aligned}
\mathbf{X}_{l, v}^{(\omega)}\left(s_{1}, s_{2}\right):=\frac{1}{4\left|\Lambda_{l}\right|} \sum_{\mathbf{x}, \mathbf{y} \in \mathfrak{K}} \int_{v}^{\beta-v} \mathrm{~d} \alpha\left(\mathfrak{C}_{s_{1}-s_{2}+i \alpha}^{(\omega)}(\mathbf{x}, \mathbf{y})-\mathfrak{C}_{i \alpha}^{(\omega)}(\mathbf{x}, \mathbf{y})\right) \\
\times \mathbf{E}_{s_{1}}^{\mathbf{A}_{l}}(\mathbf{x}) \mathbf{E}_{s_{2}}^{\mathbf{A}_{l}}(\mathbf{y}) .
\end{aligned}
$$

$\mathfrak{C}_{t+i \alpha}^{(\omega)}$ is the map from $\mathfrak{L}^{4}$ to $\mathbb{C}$ defined by

$$
\mathfrak{C}_{t+i \alpha}^{(\omega)}(\mathbf{x}, \mathbf{y}):=\sum_{\pi, \pi^{\prime} \in S_{2}} \varepsilon_{\pi} \varepsilon_{\pi^{\prime}} C_{t+i \alpha}^{(\omega)}\left(y^{\pi^{\prime}(1)}, x^{\pi(1)}\right) C_{-t+i(\beta-\alpha)}^{(\omega)}\left(x^{\pi(2)}, y^{\pi^{\prime}(2)}\right)
$$

for any $\mathbf{x}:=\left(x^{(1)}, x^{(2)}\right) \in \mathfrak{L}^{2}$ and $\mathbf{y}:=\left(y^{(1)}, y^{(2)}\right) \in \mathfrak{L}^{2}$, where $\pi, \pi^{\prime} \in S_{2}$ are by definition permutations of $\{1,2\}$ with signatures $\varepsilon_{\pi}, \varepsilon_{\pi^{\prime}} \in\{-1,1\}$. The definition of the set $\mathfrak{K} \subset \mathfrak{L}^{2}$ of bonds of nearest neighbors is given by (104). Note also that the integral in (121) can be exchanged with the (finite) sum because $\mathbf{A} \in \mathbf{C}_{0}^{\infty}$. 
The first important result of the present subsection will be a proof that $\mathbf{X}_{l, 0}^{(\omega)}$ almost surely converges to a deterministic function, as $l \rightarrow \infty$. See Corollary 5.19. Then, we will use Lebesgue's dominated convergence theorem to get the paramagnetic energy increment $\mathfrak{I}_{\mathrm{p}}^{\left(\omega, \eta \mathbf{A}_{l}\right)}$ in the limit $\left(\eta, l^{-1}\right) \rightarrow(0,0)$, see Theorem 5.21 .

By Theorem 5.1, note that, for all $\varepsilon, \beta \in \mathbb{R}^{+}, \lambda \in \mathbb{R}_{0}^{+}, t \in \mathbb{R}, v \in(0, \beta / 2)$ and $\alpha \in[v, \beta-v]$, the complex-time two-point correlation function $C_{t+i \alpha}^{(\omega)}$ can be written as the sum

$$
C_{t+i \alpha}^{(\omega)}(\mathbf{x})=A_{t+i \alpha, v, \varepsilon}^{(\omega)}(\mathbf{x})+B_{t+i \alpha, v, \varepsilon}^{(\omega)}(\mathbf{x}), \quad \mathbf{x}:=\left(x^{(1)}, x^{(2)}\right) \in \mathfrak{L}^{2},
$$

of two maps $A_{t+i \alpha, v, \varepsilon}^{(\omega)}, B_{t+i \alpha, v, \varepsilon}^{(\omega)}$ from $\mathfrak{L}^{2}$ to $\mathbb{C}$. This decomposition has the following useful property: $A_{t+i \alpha, v, \varepsilon}^{(\omega)}$ can be seen as the kernel (w.r.t. the canonical basis $\left.\left\{\mathfrak{e}_{x}\right\}_{x \in \mathfrak{L}}\right)$ of an operator, again denoted by $A_{t+i \alpha, v, \varepsilon}^{(\omega)} \in \mathcal{B}\left(\ell^{2}(\mathfrak{L})\right)$, with arbitrarily small operator norm $\left\|A_{t+i \alpha, v, \varepsilon}^{(\omega)}\right\|_{\text {op }} \leq \varepsilon$, whereas $B_{t+i \alpha, v, \varepsilon}^{(\omega)}(\mathbf{x})$ rapidly decays, as $\left|x^{(1)}-x^{(2)}\right| \rightarrow \infty$. This is however only satisfied if $\alpha \in[v, \beta-v]$ with fixed $v \in(0, \beta / 2)$, see Theorem 5.1 .

As a consequence, the first step is to approximate $\mathbf{X}_{l, 0}^{(\omega)}$ with $\mathbf{X}_{l, v}^{(\omega)}$ for arbitrarily small parameters $v>0$ :

\section{Lemma 5.10 (Approximation I)}

Let $\mathbf{A} \in \mathbf{C}_{0}^{\infty}$. Then,

$$
\mathbf{X}_{l, 0}^{(\omega)}\left(s_{1}, s_{2}\right)=\mathbf{X}_{l, v}^{(\omega)}\left(s_{1}, s_{2}\right)+\mathcal{O}(v),
$$

uniformly for $l, \beta \in \mathbb{R}^{+}, \omega \in \Omega, \lambda \in \mathbb{R}_{0}^{+}$and $s_{1}, s_{2} \in \mathbb{R}$.

Proof: The canonical orthonormal basis of $\ell^{2}(\mathfrak{L}) \otimes \ell^{2}(\mathfrak{L})$ is defined by $\left\{\mathbf{e}_{\mathbf{x}}\right\}_{\mathbf{x} \in \mathfrak{L}^{2}}$ with

$$
\mathbf{e}_{\mathbf{x}}:=\mathfrak{e}_{x^{(1)}} \otimes \mathfrak{e}_{x^{(2)}}, \quad \mathbf{x}:=\left(x^{(1)}, x^{(2)}\right) \in \mathfrak{L}^{2} .
$$

Recall that $\mathfrak{e}_{x}(y) \equiv \delta_{x, y} \in \ell^{2}(\mathfrak{L})$. The coefficient $\mathfrak{C}_{t+i \alpha}^{(\omega)}$ defined by (122) can be seen as the kernel (w.r.t. $\left\{\mathbf{e}_{\mathbf{x}}\right\}_{\mathbf{x} \in \mathfrak{L}^{2}}$ ) of a bounded operator on $\ell^{2}(\mathfrak{L}) \otimes \ell^{2}(\mathfrak{L})$ that is again denoted by $\mathfrak{C}_{t+i \alpha}^{(\omega)}$. In particular, similar to (85),

$$
\sum_{\mathbf{x}, \mathbf{y} \in \mathfrak{K}} \mathfrak{C}_{s_{1}-s_{2}+i \alpha}^{(\omega)}(\mathbf{x}, \mathbf{y}) \mathbf{E}_{s_{1}}^{\mathbf{A}_{l}}(\mathbf{x}) \mathbf{E}_{s_{2}}^{\mathbf{A}_{l}}(\mathbf{y})=\sum_{\mathbf{x}, \mathbf{y} \in \mathfrak{K}}\left\langle\mathbf{e}_{\mathbf{y}}, \mathfrak{C}_{s_{1}-s_{2}+i \alpha}^{(\omega)} \mathbf{e}_{\mathbf{x}}\right\rangle \mathbf{E}_{s_{1}}^{\mathbf{A}_{l}}(\mathbf{x}) \mathbf{E}_{s_{2}}^{\mathbf{A}_{l}}(\mathbf{y}) .
$$


In particular, via [BPK2, Lemma 5.3], i.e., $\left\|\mathfrak{C}_{t+i \alpha}^{(\omega)}\right\|_{\text {op }} \leq 4$, and Equation (108) we arrive at the upper bound

$$
\left|\frac{1}{4\left|\Lambda_{l}\right|} \sum_{\mathbf{x}, \mathbf{y} \in \mathfrak{K}} \mathfrak{C}_{s_{1}-s_{2}+i \alpha}^{(\omega)}(\mathbf{x}, \mathbf{y}) \mathbf{E}_{s_{1}}^{\mathbf{A}_{l}}(\mathbf{x}) \mathbf{E}_{s_{2}}^{\mathbf{A}_{l}}(\mathbf{y})\right| \leq 2 d\left\|\mathbf{E}^{\mathbf{A}}\right\|_{\infty}^{2} \max _{t \in \mathbb{R}}|\operatorname{supp}(\mathbf{A}(t, .))|
$$

for any $\mathbf{A} \in \mathbf{C}_{0}^{\infty}, l, \beta \in \mathbb{R}^{+}, \omega \in \Omega, \lambda \in \mathbb{R}_{0}^{+}, \alpha \in[0, \beta]$ and $s_{1}, s_{2} \in \mathbb{R}$. Therefore, the assertion follows from (121) combined with (126).

Because of (123) and Theorem 5.1, it is natural to define, at any $\varepsilon, \beta \in \mathbb{R}^{+}$, $t \in \mathbb{R}, v \in(0, \beta / 2)$ and $\alpha \in[v, \beta-v]$, the map $\mathfrak{B}_{t+i \alpha, v, \varepsilon}^{(\omega)}$ from $\mathfrak{L}^{4}$ to $\mathbb{C}$ by

$$
\mathfrak{B}_{t+i \alpha, v, \varepsilon}^{(\omega)}(\mathbf{x}, \mathbf{y}):=\sum_{\pi, \pi^{\prime} \in S_{2}} \varepsilon_{\pi} \varepsilon_{\pi^{\prime}} B_{t+i \alpha, v, \varepsilon}^{(\omega)}\left(y^{\pi^{\prime}(1)}, x^{\pi(1)}\right) B_{-t+i(\beta-\alpha), v, \varepsilon}^{(\omega)}\left(x^{\pi(2)}, y^{\pi^{\prime}(2)}\right)
$$

for any $\mathbf{x}:=\left(x^{(1)}, x^{(2)}\right) \in \mathfrak{L}^{2}$ and $\mathbf{y}:=\left(y^{(1)}, y^{(2)}\right) \in \mathfrak{L}^{2}$. In other words, this map is defined by replacing in (122) the complex-time two-point correlation function $C_{t+i \alpha}^{(\omega)}$ by its approximation $B_{t+i \alpha, v, \varepsilon}^{(\omega)}$, which comes from the decomposition (123). Similarly, for $s_{1}, s_{2} \in \mathbb{R}$, let

$$
\begin{aligned}
\mathbf{Y}_{l, v, \varepsilon, 0}^{(\omega)}\left(s_{1}, s_{2}\right):=\frac{1}{4\left|\Lambda_{l}\right|} \sum_{\mathbf{x}, \mathbf{y} \in \mathfrak{K}} \int_{v}^{\beta-v} \mathrm{~d} \alpha\left(\mathfrak{B}_{s_{1}-s_{2}+i \alpha, v, \varepsilon}^{(\omega)}\right. & \left.(\mathbf{x}, \mathbf{y})-\mathfrak{B}_{i \alpha, v, \varepsilon}^{(\omega)}(\mathbf{x}, \mathbf{y})\right) \\
& \times \mathbf{E}_{s_{1}}^{\mathbf{A}_{l}}(\mathbf{x}) \mathbf{E}_{s_{2}}^{\mathbf{A}_{l}}(\mathbf{y}) .
\end{aligned}
$$

We show in the next lemma that it is a good approximation of (121), provided $v>0$.

\section{Lemma 5.11 (Approximation II)}

Let $\varepsilon, \beta \in \mathbb{R}^{+}, \mathbf{A} \in \mathbf{C}_{0}^{\infty}$ and $v \in(0, \beta / 2)$. Then,

$$
\mathbf{X}_{l, v}^{(\omega)}\left(s_{1}, s_{2}\right)=\mathbf{Y}_{l, v, \varepsilon, 0}^{(\omega)}\left(s_{1}, s_{2}\right)+\mathcal{O}(\varepsilon)
$$

uniformly for $l \in \mathbb{R}^{+}, \omega \in \Omega, \lambda \in \mathbb{R}_{0}^{+}$and $s_{1}, s_{2} \in \mathbb{R}$.

Proof: $\quad$ Let $\varepsilon, \beta \in \mathbb{R}^{+}, \omega \in \Omega, \lambda \in \mathbb{R}_{0}^{+}, \mathbf{A} \in \mathbf{C}_{0}^{\infty}$ and $v \in(0, \beta / 2)$. By Theorem 5.1 (i) and (85), $A_{t+i \alpha, v, \varepsilon}^{(\omega)}, B_{t+i \alpha, v, \varepsilon}^{(\omega)}$ can be seen as the kernels (w.r.t. $\left\{\mathfrak{e}_{x}\right\}_{x \in \mathfrak{L}}$ ) of two bounded operators on $\ell^{2}(\mathfrak{L})$. Therefore, Theorem 5.1 (i) and the CauchySchwarz inequality yield the existence of a finite constant $D \in \mathbb{R}^{+}$depending on 
$\beta, v$ but not on $\varepsilon \in \mathbb{R}^{+}, \omega \in \Omega, \lambda \in \mathbb{R}_{0}^{+}, \alpha \in[v, \beta-v]$ and $t \in \mathbb{R}$ such that, for all $c_{x}, c_{y}^{\prime} \in \mathbb{C}, x, y \in \mathfrak{L}$,

$$
\begin{aligned}
\left|\sum_{x, y \in \mathfrak{L}} \overline{c_{x}} c_{y}^{\prime} A_{t+i \alpha, v, \varepsilon}^{(\omega)}(x, y)\right| & \leq\left\|A_{t+i \alpha, v, \varepsilon}^{(\omega)}\right\| \|_{\mathrm{op}} \sqrt{\sum_{x, y \in \mathfrak{L}}\left|c_{x}\right|^{2}\left|c_{y}^{\prime}\right|^{2}} \\
& \leq \varepsilon \sqrt{\sum_{x, y \in \mathfrak{L}}\left|c_{x}\right|^{2}\left|c_{y}^{\prime}\right|^{2}}, \\
\left|\sum_{x, y \in \mathfrak{L}} \overline{c_{x}} c_{y}^{\prime} B_{t+i \alpha, v, \varepsilon}^{(\omega)}(x, y)\right| & \leq\left\|B_{t+i \alpha, v, \varepsilon}^{(\omega)}\right\|_{\mathrm{op}} \sqrt{\sum_{x, y \in \mathfrak{L}}\left|c_{x}\right|^{2}\left|c_{y}^{\prime}\right|^{2}} \\
& \leq D \sqrt{\sum_{x, y \in \mathfrak{L}}\left|c_{x}\right|^{2}\left|c_{y}^{\prime}\right|^{2}} .
\end{aligned}
$$

It obviously follows that, for all $c_{\mathbf{x}} \in \mathbb{C}, \mathbf{x} \in \mathfrak{K}$, and some similar constant $D \in \mathbb{R}^{+}$,

$$
\begin{aligned}
& \left|\sum_{\mathbf{x}, \mathbf{y} \in \mathfrak{K}} \overline{c_{\mathbf{x}}} c_{\mathbf{y}} B_{t+i \alpha, v, \varepsilon}^{(\omega)}\left(y^{(1)}, x^{(1)}\right) A_{-t+i(\beta-\alpha), v, \varepsilon}^{(\omega)}\left(x^{(2)}, y^{(2)}\right)\right| \leq \varepsilon D \sum_{\mathbf{x} \in \mathfrak{K}}\left|c_{\mathbf{x}}\right|^{2}, \\
& \left|\sum_{\mathbf{x}, \mathbf{y} \in \mathfrak{K}} \overline{c_{\mathbf{x}}} c_{\mathbf{y}} A_{t+i \alpha, v, \varepsilon}^{(\omega)}\left(y^{(1)}, x^{(1)}\right) B_{-t+i(\beta-\alpha), v, \varepsilon}^{(\omega)}\left(x^{(2)}, y^{(2)}\right)\right| \leq \varepsilon D \sum_{\mathbf{x} \in \mathfrak{K}}\left|c_{\mathbf{x}}\right|^{2}, \\
& \left|\sum_{\mathbf{x}, \mathbf{y} \in \mathfrak{K}} \overline{c_{\mathbf{x}}} c_{\mathbf{y}} A_{t+i \alpha, v, \varepsilon}^{(\omega)}\left(y^{(1)}, x^{(1)}\right) A_{-t+i(\beta-\alpha), v, \varepsilon}^{(\omega)}\left(x^{(2)}, y^{(2)}\right)\right| \leq \varepsilon^{2} D \sum_{\mathbf{x} \in \mathfrak{K}}\left|c_{\mathbf{x}}\right|^{2},
\end{aligned}
$$

provided $\alpha \in[v, \beta-v]$ with $v \in(0, \beta / 2)$. Here, $\mathbf{x}=\left(x^{(1)}, x^{(2)}\right), \mathbf{y}=\left(y^{(1)}, y^{(2)}\right)$. Similar to (126), we then use these three above bounds to get the existence of a finite constant $D \in \mathbb{R}^{+}$depending on $\beta, v, \mathbf{A}$ but not on $l \in \mathbb{R}^{+}, \varepsilon \in(0,1)$, $\omega \in \Omega, \lambda \in \mathbb{R}_{0}^{+}$and $s_{1}, s_{2} \in \mathbb{R}$ such that

$$
\left|\mathbf{X}_{l, v}^{(\omega)}\left(s_{1}, s_{2}\right)-\mathbf{Y}_{l, v, \varepsilon, 0}^{(\omega)}\left(s_{1}, s_{2}\right)\right| \leq \varepsilon D
$$

The approximating correlation functions $B_{t+i \alpha, v, \varepsilon}^{(\omega)}$ in (127) rapidly vanish, as $\left|y^{\pi^{\prime}(1)}-x^{\pi(1)}\right| \rightarrow \infty$ or $\left|x^{\pi(2)}-y^{\pi^{\prime}(2)}\right| \rightarrow \infty$, see Theorem 5.1 (ii). The decay is uniform for times $t$ on compact sets. This property will allows us further on to use piecewise-constant approximations of the smooth electric field $E_{\mathbf{A}}$ (12) in (128), similar to what is done in the preceding subsection. 
To do this, as in the proof of Theorem 5.8, let us assume w.l.o.g. that, for all $t \in \mathbb{R}$,

$$
\operatorname{supp}(\mathbf{A}(t, .)) \subset[-1 / 2,1 / 2]^{d} .
$$

For all $n \in \mathbb{N}$, we divide the elementary box $[-1 / 2,1 / 2]^{d}$ in $n^{d}$ boxes $\left\{b_{j}\right\}_{j \in \mathcal{D}_{n}}$ of side-length $1 / n$. The sets $\mathcal{D}_{n}$ and $\left\{b_{j}\right\}_{j \in \mathcal{D}_{n}}$ are defined by (93)-(94), respectively. Then, for all $\varepsilon, l, \beta \in \mathbb{R}^{+}, \omega \in \Omega, \lambda \in \mathbb{R}_{0}^{+}$and $v \in(0, \beta / 2)$, we extend the definition of $\mathbf{Y}_{l, v, \varepsilon, 0}^{(\omega)}$ to all $n \in \mathbb{N}$ as

$$
\begin{aligned}
\mathbf{Y}_{l, v, \varepsilon, n}^{(\omega)}\left(s_{1}, s_{2}\right):=\frac{1}{4\left|\Lambda_{l}\right|} & \sum_{j \in \mathcal{D}_{n}} \sum_{\mathbf{x}, \mathbf{y} \in \mathfrak{K} \cap\left(l b_{j}\right)^{2}} \int_{v}^{\beta-v} \mathrm{~d} \alpha \\
& \times\left(\mathfrak{B}_{s_{1}-s_{2}+i \alpha, v, \varepsilon}^{(\omega)}(\mathbf{x}, \mathbf{y})-\mathfrak{B}_{i \alpha, v, \varepsilon}^{(\omega)}(\mathbf{x}, \mathbf{y})\right) \mathbf{E}_{s_{1}}^{\mathbf{A}_{l}}(\mathbf{x}) \mathbf{E}_{s_{2}}^{\mathbf{A}_{l}}(\mathbf{y})
\end{aligned}
$$

for all $s_{1}, s_{2} \in \mathbb{R}$. In fact, the accumulation points of $\mathbf{Y}_{l, v, \varepsilon, n}^{(\omega)}$, as $l \rightarrow \infty$, do not depend on $n$ :

\section{Lemma 5.12 (Approximation III)}

Let $n \in \mathbb{N}, \varepsilon, \beta \in \mathbb{R}^{+}, \omega \in \Omega, \lambda \in \mathbb{R}_{0}^{+}$and $v \in(0, \beta / 2)$. Then,

$$
\lim _{l \rightarrow \infty}\left|\mathbf{Y}_{l, v, \varepsilon, 0}^{(\omega)}\left(s_{1}, s_{2}\right)-\mathbf{Y}_{l, v, \varepsilon, n}^{(\omega)}\left(s_{1}, s_{2}\right)\right|=0
$$

uniformly for $s_{1}, s_{2} \in \mathbb{R}$.

Proof: We observe from (94) and (128)-(129) that

$$
\begin{aligned}
& \left|\mathbf{Y}_{l, v, \varepsilon, 0}^{(\omega)}\left(s_{1}, s_{2}\right)-\mathbf{Y}_{l, v, \varepsilon, n}^{(\omega)}\left(s_{1}, s_{2}\right)\right| \\
\leq & \frac{1}{4\left|\Lambda_{l}\right|} \sum_{j, k \in \mathcal{D}_{n}, j \neq k} \sum_{\mathbf{x} \in \mathfrak{K} \cap\left(l b_{j}\right)^{2}} \sum_{\mathbf{y} \in \mathfrak{K} \cap\left(l b_{k}\right)^{2}}\left|\mathbf{K}_{s_{1}, s_{2}}(\mathbf{x}, \mathbf{y})\right| \\
& +\frac{1}{4\left|\Lambda_{l}\right|} \sum_{j \in \mathcal{D}_{n}} \sum_{\mathbf{x} \in \partial\left(l b_{j}\right)} \sum_{\mathbf{y} \in \mathfrak{K}}\left|\mathbf{K}_{s_{1}, s_{2}}(\mathbf{x}, \mathbf{y})+\mathbf{K}_{s_{1}, s_{2}}(\mathbf{y}, \mathbf{x})\right|,
\end{aligned}
$$

where, for any $\Lambda \in \mathcal{P}_{f}(\mathfrak{L})$ with complement $\Lambda^{c} \subset \mathfrak{L}$,

$$
\partial \Lambda:=\left\{\left(x^{(1)}, x^{(2)}\right) \in \mathfrak{K}:\left\{x^{(1)}, x^{(2)}\right\} \cap \Lambda \neq 0,\left\{x^{(1)}, x^{(2)}\right\} \cap \Lambda^{c} \neq 0\right\}
$$

and

$\mathbf{K}_{s_{1}, s_{2}}(\mathbf{x}, \mathbf{y}):=\int_{v}^{\beta-v} \mathrm{~d} \alpha\left(\mathfrak{B}_{s_{1}-s_{2}+i \alpha, v, \varepsilon}^{(\omega)}(\mathbf{x}, \mathbf{y})-\mathfrak{B}_{i \alpha, v, \varepsilon}^{(\omega)}(\mathbf{x}, \mathbf{y})\right) \mathbf{E}_{s_{1}}^{\mathbf{A}_{l}}(\mathbf{x}) \mathbf{E}_{s_{2}}^{\mathbf{A}_{l}}(\mathbf{y})$. 
Meanwhile, for any $j, k \in \mathcal{D}_{n}, j \neq k$, and every

$$
\mathbf{x}:=\left(x^{(1)}, x^{(2)}\right) \in \mathfrak{K}, \quad \mathbf{y}:=\left(y^{(1)}, y^{(2)}\right) \in \mathfrak{K},
$$

one clearly has the lower bound

$$
\min _{\pi, \pi^{\prime} \in S_{2}}\left|x^{\pi(1)}-y^{\pi^{\prime}(1)}\right| \geq \min \left\{|| x^{(1)}-y^{(1)}|-2|,\left|x^{(1)}-y^{(1)}\right|\right\},
$$

see (104). We use this simple inequality together with (108) and Theorem 5.1 (ii) to obtain from (127) and (130) that, for all $s_{1}, s_{2} \in \mathbb{R}$,

$$
\begin{aligned}
& \left|\mathbf{Y}_{l, v, \varepsilon, 0}^{(\omega)}\left(s_{1}, s_{2}\right)-\mathbf{Y}_{l, v, \varepsilon, n}^{(\omega)}\left(s_{1}, s_{2}\right)\right| \\
\leq & \frac{D}{l^{d}} \sum_{j, k \in \mathcal{D}_{n}, j \neq k} \sum_{x \in \mathfrak{L} \cap\left(l b_{j}\right)} \sum_{y \in \mathfrak{L} \cap\left(l b_{k}\right)} \frac{1}{(1+|x-y|)^{2 d^{2}+2}} \\
& +\frac{D}{l^{d}} \sum_{j \in \mathcal{D}_{n}} \sum_{\left(x^{(1)}, x^{(2)}\right) \in \partial\left(l b_{j}\right)} \sum_{y \in \mathfrak{L}} \frac{1}{\left(1+\left|x^{(1)}-y\right|\right)^{2 d^{2}+2}},
\end{aligned}
$$

where $D \in \mathbb{R}^{+}$is a finite constant only depending on $\varepsilon, \beta, v, d$ and $\mathbf{A} \in \mathbf{C}_{0}^{\infty}$. Note that the second term of the r.h.s. of the above inequality is of order $\mathcal{O}\left(l^{-1}\right)$. For any small $\delta>0$ with $\delta l \geq 1$,

$$
\frac{1}{l^{d}} \sum_{j, k \in \mathcal{D}_{n}, j \neq k} \sum_{x \in \mathfrak{L} \cap\left(l b_{j}\right)} \sum_{y \in \mathfrak{L} \cap\left(l b_{k}\right)} \frac{\mathbf{1}[|x-y| \geq \delta l]}{(1+|x-y|)^{2 d^{2}+2}}=\mathcal{O}\left(\frac{1}{l^{2 d^{2}-d+2} \delta^{2 d^{2}+2}}\right)
$$

and

$$
\frac{1}{l^{d}} \sum_{j, k \in \mathcal{D}_{n}, j \neq k} \sum_{x \in \mathfrak{L} \cap\left(l b_{j}\right)} \sum_{y \in \mathfrak{L} \cap\left(l b_{k}\right)} \frac{\mathbf{1}[|x-y| \leq \delta l]}{(1+|x-y|)^{2 d^{2}+2}}=\mathcal{O}\left(\delta^{d+1} l^{d}\right) .
$$

Then, by choosing $\delta=l^{-\frac{2 d^{2}+2}{2 d^{2}+d+3}}$, the last two sums are both of order $\mathcal{O}\left(l^{-\frac{d^{2}-d+2}{2 d^{2}+d+3}}\right)$ with $d^{2}-d+2 \geq 2$ for all $d \in \mathbb{N}$. Using this estimate to bound the first term of the r.h.s. of (131) we arrive at the assertion.

As already mentioned above, we now consider piecewise-constant approximations of the (smooth) electric field $E_{\mathbf{A}}(111)$. For any $j \in \mathcal{D}_{n}$, let $z^{(j)} \in b_{j}$ be 
any fixed point of the box $b_{j}$. Then, for any $s_{1}, s_{2} \in \mathbb{R}$, define the function

$$
\begin{aligned}
\overline{\mathbf{Y}}_{l, v, \varepsilon, n}^{(\omega)}\left(s_{1}, s_{2}\right):=\frac{1}{4\left|\Lambda_{l}\right|} & \sum_{j \in \mathcal{D}_{n}} \sum_{\mathbf{x}, \mathbf{y} \in \mathfrak{K} \cap\left(l b_{j}\right)^{2}} \int_{v}^{\beta-v} \mathrm{~d} \alpha \\
& \times\left(\mathfrak{B}_{s_{1}-s_{2}+i \alpha, v, \varepsilon}^{(\omega)}(\mathbf{x}, \mathbf{y})-\mathfrak{B}_{i \alpha, v, \varepsilon}^{(\omega)}(\mathbf{x}, \mathbf{y})\right) \\
& \times\left[E_{\mathbf{A}}\left(s_{1}, z^{(j)}\right)\right]\left(x^{(2)}-x^{(1)}\right)\left[E_{\mathbf{A}}\left(s_{2}, z^{(j)}\right)\right]\left(y^{(2)}-y^{(1)}\right) .
\end{aligned}
$$

Recall that $\mathbf{x}:=\left(x^{(1)}, x^{(2)}\right) \in \mathfrak{L}^{2}$ and $\mathbf{y}:=\left(y^{(1)}, y^{(2)}\right) \in \mathfrak{L}^{2}$. See also (104). This new function approximates (129) arbitrarily well, as $l \rightarrow \infty$ and $n \rightarrow \infty$ :

\section{Lemma 5.13 (Approximation IV)}

Let $n \in \mathbb{N}, \varepsilon, l, \beta \in \mathbb{R}^{+}, \omega \in \Omega, \lambda \in \mathbb{R}_{0}^{+}$and $v \in(0, \beta / 2)$. Then,

$$
\lim _{n \rightarrow \infty}\left\{\limsup _{l \rightarrow \infty}\left|\mathbf{Y}_{l, v, \varepsilon, n}^{(\omega)}\left(s_{1}, s_{2}\right)-\overline{\mathbf{Y}}_{l, v, \varepsilon, n}^{(\omega)}\left(s_{1}, s_{2}\right)\right|\right\}=0
$$

uniformly for $s_{1}, s_{2} \in \mathbb{R}$.

Proof: $\quad$ Using (108), (113) and Theorem 5.1 (ii) as in (131), one gets that, for any $s_{1}, s_{2} \in \mathbb{R}$,

$$
\begin{aligned}
& \left|\mathbf{Y}_{l, v, \varepsilon, n}^{(\omega)}\left(s_{1}, s_{2}\right)-\overline{\mathbf{Y}}_{l, v, \varepsilon, n}^{(\omega)}\left(s_{1}, s_{2}\right)\right| \\
\leq & D\left(n^{-1}+l^{-1}\right) \frac{1}{l^{d}} \sum_{j \in \mathcal{D}_{n}} \sum_{x, y \in \mathfrak{L} \cap\left(l b_{j}\right)} \frac{1}{(1+|x-y|)^{2 d^{2}+2}},
\end{aligned}
$$

where $D \in \mathbb{R}^{+}$is a finite constant only depending on $\varepsilon, \beta, v, d$ and $\mathbf{A} \in \mathbf{C}_{0}^{\infty}$. For all $j \in \mathcal{D}_{n}$ and $l>1$, note that

$$
\frac{1}{l^{d}} \sum_{x, y \in \mathfrak{L} \cap\left(l b_{j}\right)} \frac{1}{(1+|x-y|)^{2 d^{2}+2}} \leq \frac{(2 l+1)^{d}}{n^{d} l^{d}} \sum_{x \in \mathfrak{L}} \frac{1}{(1+|x|)^{2 d^{2}+2}} \leq \frac{D}{n^{d}}
$$

for some finite constant $D \in \mathbb{R}^{+}$. Therefore, we arrive at the assertion by combining this last bound with (133).

By taking the canonical orthonormal basis $\left\{e_{k}\right\}_{k=1}^{d}$ of $\mathbb{R}^{d}$ and setting $e_{-k}:=$ $-e_{k}$ for each $k \in\{1, \ldots, d\}$, we rewrite the function (132) as

$$
\begin{aligned}
& \overline{\mathbf{Y}}_{l, v, \varepsilon, n}^{(\omega)}\left(s_{1}, s_{2}\right)=\frac{1}{4 n^{d}} \sum_{j \in \mathcal{D}_{n}} \sum_{k, q \in\{1,-1, \ldots, d,-d\}}\left(\mathbf{Z}_{l, j, k, q}^{(\omega)}\left(s_{1}-s_{2}\right)-\mathbf{Z}_{l, j, k, q}^{(\omega)}(0)\right) \\
& \times\left[E_{\mathbf{A}}\left(s_{1}, z^{(j)}\right)\right]\left(e_{q}\right)\left[E_{\mathbf{A}}\left(s_{2}, z^{(j)}\right)\right]\left(e_{k}\right)
\end{aligned}
$$


for any $s_{1}, s_{2} \in \mathbb{R}$, where, for all $n \in \mathbb{N}, \varepsilon, l, \beta \in \mathbb{R}^{+}, \omega \in \Omega, \lambda \in \mathbb{R}_{0}^{+}, v \in$ $(0, \beta / 2), j \in \mathcal{D}_{n}, k, q \in\{1,-1, \ldots, d,-d\}$ and $t \in \mathbb{R}$,

$$
\mathbf{Z}_{l, j, k, q}^{(\omega)}(t):=\frac{n^{d}}{\left|\Lambda_{l}\right|} \sum_{x, y \in \mathfrak{L} \cap\left(l b_{j}\right)} \int_{v}^{\beta-v} \mathrm{~d} \alpha \mathfrak{B}_{t+i \alpha, v, \varepsilon}^{(\omega)}\left(x, x-e_{q}, y, y-e_{k}\right) .
$$

Notice that we have added terms related to $x, y$ on the boundary of $\mathfrak{L} \cap\left(l b_{j}\right)$, but we use the same notation $\overline{\mathbf{Y}}_{l, v, \varepsilon, n}^{(\omega)}$ for simplicity. These terms are indeed irrelevant in the limit $l \rightarrow \infty$. For $\mathbf{x}:=\left(x^{(1)}, x^{(2)}\right) \in \mathfrak{L}^{2}$ and $\mathbf{y}:=\left(y^{(1)}, y^{(2)}\right) \in \mathfrak{L}^{2}$, we used the notation

$$
\mathfrak{B}_{t+i \alpha, v, \varepsilon}^{(\omega)}\left(x^{(1)}, x^{(2)}, y^{(1)}, y^{(2)}\right) \equiv \mathfrak{B}_{t+i \alpha, v, \varepsilon}^{(\omega)}(\mathbf{x}, \mathbf{y}),
$$

see (127). By Theorem 5.1 (i), (iv) and Lebesgue's dominated convergence theorem, note that, for all $\varepsilon, \beta \in \mathbb{R}^{+}, \lambda \in \mathbb{R}_{0}^{+}, x, y \in \mathfrak{L}, k, q \in\{1,-1, \ldots, d,-d\}$, $t \in \mathbb{R}$ and $v \in(0, \beta / 2)$, the map

$$
\omega \mapsto \int_{0}^{\beta} \mathrm{d} \alpha \mathfrak{B}_{t+i \alpha, v, \varepsilon}^{(\omega)}\left(x, x-e_{q}, y, y-e_{k}\right)
$$

is bounded and measurable w.r.t. the $\sigma$-algebra $\mathfrak{A}_{\Omega}$. In particular, its expectation value $\mathbb{E}[\cdot]$ w.r.t. the probability measure $\mathfrak{a}_{\Omega}(8)$ is well-defined. It now remains to analyze the limit of $\mathbf{Z}_{l, j, k, q}^{(\omega)}$, as $l \rightarrow \infty$.

\section{Lemma 5.14 (Infinite volume limit and ergodicity)}

Let $\varepsilon, l, \beta \in \mathbb{R}^{+}, \lambda \in \mathbb{R}_{0}^{+}, t \in \mathbb{R}$ and $v \in(0, \beta / 2)$. Then, there is a measurable subset $\tilde{\Omega}_{v, \varepsilon}(t) \equiv \tilde{\Omega}_{v, \varepsilon}^{(\beta, \lambda)}(t) \subset \Omega$ of full measure such that, for any $n \in \mathbb{N}, j \in \mathcal{D}_{n}$, $k, q \in\{1,-1, \ldots, d,-d\}$ and any $\omega \in \tilde{\Omega}_{v, \varepsilon}(t)$,

$$
\lim _{l \rightarrow \infty} \mathbf{Z}_{l, j, k, q}^{(\omega)}(t)=\sum_{x \in \mathfrak{L}} \mathbb{E}\left[\int_{v}^{\beta-v} \mathrm{~d} \alpha \mathfrak{B}_{t+i \alpha, v, \varepsilon}^{(\omega)}\left(x, x-e_{q}, 0,-e_{k}\right)\right] \in \mathbb{R} .
$$

Proof: The arguments are similar to those proving Theorems 5.6 or 5.8, but a little bit more complicated. For the reader's convenience, we give the proof in detail. For any $\varepsilon, \beta \in \mathbb{R}^{+}, \omega \in \Omega, \lambda \in \mathbb{R}_{0}^{+}, t \in \mathbb{R}, v \in(0, \beta / 2), k, q \in$ $\{1,-1, \ldots, d,-d\}$ and $y \in \mathfrak{L}$, let

$$
\mathfrak{F}_{t, v, \varepsilon, k, q}^{(\beta, \omega, \lambda)}(\{y\}):=\sum_{x \in \mathfrak{L}} \int_{v}^{\beta-v} \mathrm{~d} \alpha \mathfrak{B}_{t+i \alpha, v, \varepsilon}^{(\omega)}\left(x, x-e_{q}, y, y-e_{k}\right) \in \mathbb{R} .
$$


This infinite sum absolutely converges because of (127) and Theorem 5.1 (ii). We now define an additive process $\left\{\mathfrak{F}_{t, v, \varepsilon, k, q}^{(\beta, \omega, \lambda)}(\Lambda)\right\}_{\Lambda \in \mathcal{P}_{f}(\mathfrak{L})}$ by

$$
\mathfrak{F}_{t, v, \varepsilon, k, q}^{(\beta, \omega, \lambda)}(\Lambda):=\sum_{y \in \Lambda} \mathfrak{F}_{t, v, \varepsilon, k, q}^{(\beta, \omega, \lambda)}(\{y\})
$$

for any finite subset $\Lambda \in \mathcal{P}_{f}(\mathfrak{L})$, see Definition 5.2. Indeed, by Theorem 5.1 (i), (iv) and Lebesgue's dominated convergence theorem, the map $\omega \mapsto \mathfrak{F}_{t, v, \varepsilon, k, q}^{(\beta, \omega, \lambda)}(\Lambda)$ is bounded and measurable (in fact continuous) w.r.t. the $\sigma$-algebra $\mathfrak{A}_{\Omega}$ for all $\Lambda \in \mathcal{P}_{f}(\mathfrak{L})$. Then, for any $\varepsilon, \beta \in \mathbb{R}^{+}, \lambda \in \mathbb{R}_{0}^{+}, t \in \mathbb{R}$ and $v \in(0, \beta / 2)$, we apply Theorem 5.5 on the previous additive process to get the existence of a measurable subset

$$
\tilde{\Omega}_{v, \varepsilon}(t) \equiv \tilde{\Omega}_{v, \varepsilon}^{(\beta, \lambda)}(t) \subset \Omega
$$

of full measure such that, for all $\omega \in \tilde{\Omega}_{v, \varepsilon}(t), n \in \mathbb{N}, j \in \mathcal{D}_{n}$ and $k, q \in$ $\{1,-1, \ldots, d,-d\}$,

$$
\lim _{l \rightarrow \infty}\left\{\frac{n^{d}}{\left|\Lambda_{l}\right|} \mathfrak{F}_{t, v, \varepsilon, k, q}^{(\beta, \omega)}\left(l b_{j}\right)\right\}=\mathbb{E}\left[\mathfrak{F}_{t, v, \varepsilon, k, q}^{(\beta, \omega)}(\{0\})\right] .
$$

Note that to prove this equation we use once again that any countable intersection of measurable sets of full measure has full measure. In the way one proves Lemma 5.12 , one verifies that

$$
\lim _{l \rightarrow \infty}\left\{\frac{n^{d}}{\left|\Lambda_{l}\right|} \sum_{y \in \mathfrak{L} \cap\left(l b_{j}\right)} \sum_{x \in \mathfrak{L} \backslash\left(l b_{j}\right)} \int_{v}^{\beta-v} \mathrm{~d} \alpha \mathfrak{B}_{t+i \alpha, v, \varepsilon}^{(\omega)}\left(x, x-e_{q}, y, y-e_{k}\right)\right\}=0 .
$$

Using this with (136)-(137) and observing that

$$
\mathbb{E}\left[\mathfrak{F}_{t, v, \varepsilon, k, q}^{(\beta, \omega, \lambda)}(\{0\})\right]=\sum_{x \in \mathfrak{L}} \mathbb{E}\left[\int_{v}^{\beta-v} \mathrm{~d} \alpha \mathfrak{B}_{t+i \alpha, v, \varepsilon}^{(\omega)}\left(x, x-e_{q}, 0,-e_{k}\right)\right],
$$

we arrive at the assertion for any realization $\omega \in \tilde{\Omega}_{v, \varepsilon}(t)$.

For all $\varepsilon, \beta \in \mathbb{R}^{+}, \lambda \in \mathbb{R}_{0}^{+}, v \in(0, \beta / 2)$ and $k, q \in\{1,-1, \ldots, d,-d\}$, define the functions

$$
\tilde{\Gamma}_{v, \varepsilon, k, q}(t):=\sum_{x \in \mathfrak{L}} \mathbb{E}\left[\int_{v}^{\beta-v} \mathrm{~d} \alpha \mathfrak{B}_{t+i \alpha, v, \varepsilon}^{(\omega)}\left(x, x-e_{q}, 0,-e_{k}\right)\right]
$$


for any $t \in \mathbb{R}$, and

$$
\begin{aligned}
\mathbf{Y}_{\infty, v, \varepsilon}\left(s_{1}, s_{2}\right):= & \sum_{k, q \in\{1,-1, \ldots, d,-d\}}\left(\tilde{\Gamma}_{v, \varepsilon, k, q}\left(s_{1}-s_{2}\right)-\tilde{\Gamma}_{v, \varepsilon, k, q}(0)\right) \\
& \times \int_{\mathbb{R}^{d}} \mathrm{~d}^{d} x\left[E_{\mathbf{A}}\left(s_{1}, x\right)\right]\left(e_{q}\right)\left[E_{\mathbf{A}}\left(s_{2}, x\right)\right]\left(e_{k}\right)
\end{aligned}
$$

for any $s_{1}, s_{2} \in \mathbb{R}$. We show next that the function $\mathbf{Y}_{l, v, \varepsilon, 0}^{(\omega)}$ defined by (128) almost surely converges to the deterministic function $\mathbf{Y}_{\infty, v, \varepsilon}$, as $l \rightarrow \infty$ :

\section{Proposition 5.15 (Infinite volume limit of the $\mathbf{Y}$-approximation)}

Let $\varepsilon, \beta \in \mathbb{R}^{+}, \lambda \in \mathbb{R}_{0}^{+}, v \in(0, \beta / 2)$ and $s_{1}, s_{2} \in \mathbb{R}$. Then, there is a measurable subset $\tilde{\Omega}_{v, \varepsilon}\left(s_{1}, s_{2}\right) \equiv \tilde{\Omega}_{v, \varepsilon}^{(\beta, \lambda)}\left(s_{1}, s_{2}\right) \subset \Omega$ of full measure such that, for any $\mathbf{A} \in$ $\mathbf{C}_{0}^{\infty}$ and $\omega \in \tilde{\Omega}_{v, \varepsilon}\left(s_{1}, s_{2}\right)$,

$$
\lim _{l \rightarrow \infty} \mathbf{Y}_{l, v, \varepsilon, 0}^{(\omega)}\left(s_{1}, s_{2}\right)=\mathbf{Y}_{\infty, v, \varepsilon}\left(s_{1}, s_{2}\right)
$$

Proof: $\quad$ Let $\varepsilon, \beta \in \mathbb{R}^{+}, \lambda \in \mathbb{R}_{0}^{+}, v \in(0, \beta / 2), \mathbf{A} \in \mathbf{C}_{0}^{\infty}$ and $s_{1}, s_{2} \in \mathbb{R}$. Using Lemmata 5.12-5.14 and (134), we obtain the existence of a measurable subset $\tilde{\Omega}_{v, \varepsilon}\left(s_{1}, s_{2}\right) \equiv \tilde{\Omega}_{v, \varepsilon}^{(\beta, \lambda)}\left(s_{1}, s_{2}\right) \subset \Omega$ of full measure such that, for any $\omega \in$ $\tilde{\Omega}_{v, \varepsilon}\left(s_{1}, s_{2}\right)$,

$$
\begin{aligned}
\lim _{l \rightarrow \infty} \mathbf{Y}_{l, v, \varepsilon, 0}^{(\omega)}\left(s_{1}, s_{2}\right) & =\sum_{k, q \in\{1,-1, \ldots, d,-d\}}\left(\tilde{\Gamma}_{v, \varepsilon, k, q}\left(s_{1}-s_{2}\right)-\tilde{\Gamma}_{v, \varepsilon, k, q}(0)\right) \\
\times & \lim _{n \rightarrow \infty}\left\{\frac{1}{4 n^{d}} \sum_{j \in \mathcal{D}_{n}}\left[E_{\mathbf{A}}\left(s_{1}, z^{(j)}\right)\right]\left(e_{q}\right)\left[E_{\mathbf{A}}\left(s_{2}, z^{(j)}\right)\right]\left(e_{k}\right)\right\} .
\end{aligned}
$$

The latter implies the proposition because the term within the limit $n \rightarrow \infty$ is a Riemann sum and $E_{\mathbf{A}} \in \mathbf{C}_{0}^{\infty}$ for any $\mathbf{A} \in \mathbf{C}_{0}^{\infty}$, see (111).

This last limit depends on the two arbitrary parameters $\varepsilon \in \mathbb{R}^{+}$and $v \in$ $(0, \beta / 2)$, where $\beta \in \mathbb{R}^{+}$. The next step is to remove them by considering the limits $\varepsilon \rightarrow 0^{+}$and $v \rightarrow 0^{+}$.

We first observe that the functions (138) are approximations of the function $\Gamma_{k, q} \equiv \Gamma_{k, q}^{(\beta, \lambda)}$ defined, for any $\beta \in \mathbb{R}^{+}, \lambda \in \mathbb{R}_{0}^{+}, k, q \in\{1,-1, \ldots, d,-d\}$ and $t \in \mathbb{R}$, by

$$
\Gamma_{k, q}(t):=\lim _{l \rightarrow \infty} \frac{1}{\left|\Lambda_{l}\right|} \sum_{x, y \in \Lambda_{l}} \mathbb{E}\left[\int_{0}^{\beta} \mathrm{d} \alpha \mathfrak{C}_{t+i \alpha}^{(\omega)}\left(x, x-e_{q}, y, y-e_{k}\right)\right] .
$$


By Theorem 5.1 (i), (iv) and Lebesgue's dominated convergence theorem, note that the map

$$
\omega \mapsto \int_{0}^{\beta} \mathrm{d} \alpha \mathfrak{C}_{t+i \alpha}^{(\omega)}\left(x, x-e_{q}, y, y-e_{k}\right)
$$

is bounded and measurable w.r.t. the $\sigma$-algebra $\mathfrak{A}_{\Omega}$. Here, we use the same convention for the arguments of $\mathfrak{C}_{t+i \alpha}^{(\omega)}$ as in (135) for $\mathfrak{B}_{t+i \alpha, v, \varepsilon}^{(\omega)}$. This function is well-defined and it is the limit of $\tilde{\Gamma}_{v, \varepsilon, k, q}$, as $\varepsilon \rightarrow 0^{+}$and $v \rightarrow 0^{+}$:

\section{Lemma 5.16 (Approximation on the function $\Gamma$ )}

Let $\varepsilon, \beta \in \mathbb{R}^{+}, \lambda \in \mathbb{R}_{0}^{+}, t \in \mathbb{R}, k, q \in\{1,-1, \ldots, d,-d\}$ and $v \in(0, \beta / 2)$. Then, $\Gamma_{k, q}(t)$ exists and equals

$$
\Gamma_{k, q}(t)=\tilde{\Gamma}_{v, \varepsilon, k, q}(t)+\mathcal{O}(v)+\mathcal{O}_{v}(\varepsilon)
$$

uniformly for times $t$ in compact sets. The term of order $\mathcal{O}_{v}(\varepsilon)$ vanishes when $\varepsilon \rightarrow 0^{+}$for any fixed $v \in(0, \beta / 2)$.

Proof: $\quad$ Let $\varepsilon, \beta \in \mathbb{R}^{+}, \lambda \in \mathbb{R}_{0}^{+}, v \in(0, \beta / 2), t \in \mathbb{R}$ and $k, q \in\{1,-1, \ldots, d,-d\}$. Using similar arguments to the proof of Lemma 5.11, one shows that

$$
\begin{array}{r}
\limsup _{l \rightarrow \infty} \frac{1}{\left|\Lambda_{l}\right|} \sum_{x, y \in \Lambda_{l}} \mathbb{E}\left[\int_{v}^{\beta-v} \mathrm{~d} \alpha \mid \mathfrak{B}_{t+i \alpha, v, \varepsilon}^{(\omega)}\left(x, x-e_{q}, y, y-e_{k}\right)\right. \\
\left.-\mathfrak{C}_{t+i \alpha}^{(\omega)}\left(x, x-e_{q}, y, y-e_{k}\right) \mid\right]=\mathcal{O}(\varepsilon)
\end{array}
$$

uniformly for $t \in \mathbb{R}$. Moreover, by Theorem 5.1 (ii) and translation invariance of $\mathfrak{a}_{\Omega}$ observe that, for $v \in(0, \beta / 2)$,

$$
\begin{aligned}
\lim _{l \rightarrow \infty} & \left\{\frac{1}{\left|\Lambda_{l}\right|} \sum_{x, y \in \Lambda_{l}} \mathbb{E}\left[\int_{v}^{\beta-v} \mathrm{~d} \alpha \mathfrak{B}_{t+i \alpha, v, \varepsilon}^{(\omega)}\left(x, x-e_{q}, y, y-e_{k}\right)\right]\right. \\
& \left.-\sum_{x \in \mathfrak{L}} \mathbb{E}\left[\int_{v}^{\beta-v} \mathrm{~d} \alpha \mathfrak{B}_{t+i \alpha, v, \varepsilon}^{(\omega)}\left(x, x-e_{q}, 0,-e_{k}\right)\right]\right\}=0
\end{aligned}
$$

uniformly for $t$ in compact sets. Then, one uses the same arguments as in Lemma 5.10 to obtain the assertion, see (138) and (140). We omit the details. 
We now consider the limit of the integrand $\mathbf{X}_{l, 0}^{(\omega)}$ in (120), as $l \rightarrow \infty$, and show that it converges almost surely to the deterministic function $\mathbf{X}_{\infty} \equiv \mathbf{X}_{\infty}^{(\beta, \lambda)}$ defined, for any $\beta \in \mathbb{R}^{+}, \lambda \in \mathbb{R}_{0}^{+}$and $s_{1}, s_{2} \in \mathbb{R}$, by

$$
\begin{aligned}
\mathbf{X}_{\infty}\left(s_{1}, s_{2}\right):= & \frac{1}{4} \sum_{k, q \in\{1,-1, \ldots, d,-d\}}\left(\Gamma_{k, q}\left(s_{1}-s_{2}\right)-\Gamma_{k, q}(0)\right) \\
& \times \int_{\mathbb{R}^{d}} \mathrm{~d}^{d} x\left[E_{\mathbf{A}}\left(s_{1}, x\right)\right]\left(e_{q}\right)\left[E_{\mathbf{A}}\left(s_{2}, x\right)\right]\left(e_{k}\right) .
\end{aligned}
$$

Theorem 5.17 (Infinite volume limit of the X-integrands - I)

Let $\beta \in \mathbb{R}^{+}, \lambda \in \mathbb{R}_{0}^{+}$and $s_{1}, s_{2} \in \mathbb{R}$. Then, there is a measurable subset $\tilde{\Omega}\left(s_{1}, s_{2}\right) \equiv \tilde{\Omega}^{(\beta, \lambda)}\left(s_{1}, s_{2}\right) \subset \Omega$ of full measure such that, for any $\mathbf{A} \in \mathbf{C}_{0}^{\infty}$ and $\omega \in \tilde{\Omega}\left(s_{1}, s_{2}\right)$,

$$
\lim _{l \rightarrow \infty} \mathbf{X}_{l, 0}^{(\omega)}\left(s_{1}, s_{2}\right)=\mathbf{X}_{\infty}\left(s_{1}, s_{2}\right)
$$

Proof: $\quad$ Fix $\beta \in \mathbb{R}^{+}, \lambda \in \mathbb{R}_{0}^{+}$and $s_{1}, s_{2} \in \mathbb{R}$. Define also the countable sequences $\left\{v_{n}\right\}_{n \in \mathbb{N}}$ and $\left\{\varepsilon_{m}\right\}_{m \in \mathbb{N}}$ by $v_{n}:=n^{-1}$ and $\varepsilon_{m}:=m^{-1}$ for $n, m \in$ $\mathbb{N}$. Then, by Proposition 5.15, for any $n, m \in \mathbb{N}$, there is a measurable subset $\hat{\Omega}_{n, m}\left(s_{1}, s_{2}\right) \equiv \hat{\Omega}_{n, m}^{(\beta, \lambda)}\left(s_{1}, s_{2}\right) \subset \Omega$ of full measure such that, for any $\mathbf{A} \in \mathbf{C}_{0}^{\infty}$ and $\omega \in \hat{\Omega}_{n, m}\left(s_{1}, s_{2}\right)$,

$$
\lim _{l \rightarrow \infty} \mathbf{Y}_{l, v_{n}, \varepsilon_{m}, 0}^{(\omega)}\left(s_{1}, s_{2}\right)=\mathbf{Y}_{\infty, v_{n}, \varepsilon_{m}}\left(s_{1}, s_{2}\right)
$$

Thus, we define the subset

$$
\tilde{\Omega}\left(s_{1}, s_{2}\right):=\bigcap_{n, m \in \mathbb{N}} \hat{\Omega}_{n, m}\left(s_{1}, s_{2}\right) .
$$

It has full measure, since it is a countable intersection of measurable sets of full measure.

Take $\mathbf{A} \in \mathbf{C}_{0}^{\infty}$ and any strictly positive parameter $\epsilon \in \mathbb{R}^{+}$. Then, by Lemmata 5.10, 5.11 and 5.16, there are $N_{\epsilon}, M_{\epsilon} \in \mathbb{N}$ such that, for all $l \in \mathbb{R}^{+}$and $\omega \in$ $\tilde{\Omega}\left(s_{1}, s_{2}\right)$,

$$
\left|\mathbf{X}_{l, 0}^{(\omega)}\left(s_{1}, s_{2}\right)-\mathbf{X}_{\infty}\left(s_{1}, s_{2}\right)\right| \leq \epsilon+\left|\mathbf{Y}_{l, v_{N_{\epsilon}}, \varepsilon_{M_{\epsilon}}, 0}^{(\omega)}\left(s_{1}, s_{2}\right)-\mathbf{Y}_{\infty, v_{N_{\epsilon}}, \varepsilon_{M_{\epsilon}}}\left(s_{1}, s_{2}\right)\right| .
$$

Therefore, we arrive at the assertion by combining this bound together with (143) for any realization $\omega \in \tilde{\Omega}\left(s_{1}, s_{2}\right)$. 
To find the energy increment $(120)$ in the limit $\left(\eta, l^{-1}\right) \rightarrow(0,0)$, we use below Lebesgue's dominated convergence theorem and it is crucial to remove the dependency of the measurable subset $\tilde{\Omega}\left(s_{1}, s_{2}\right)$ on $s_{1}, s_{2} \in \mathbb{R}$, see Theorem 5.17. To this end we first need to show some uniform boundedness and continuity of the function (121):

\section{Lemma 5.18 (Uniform Boundedness and Equicontinuity of X-integrands)}

Let $\beta \in \mathbb{R}^{+}, \lambda \in \mathbb{R}_{0}^{+}$and $\mathbf{A} \in \mathbf{C}_{0}^{\infty}$. The family

$$
\left\{\left(s_{1}, s_{2}\right) \mapsto \mathbf{X}_{l, 0}^{(\omega)}\left(s_{1}, s_{2}\right)\right\}_{l \in \mathbb{R}^{+}, \omega \in \Omega}
$$

of maps from $\mathbb{R}^{2}$ to $\mathbb{C}$ is uniformly bounded and equicontinuous.

Proof: The uniform boundedness of this collection of maps is an immediate consequence of (126), see (121). To prove its equicontinuity, it suffices, by Lemmata 5.10-5.11, to verify that, for any fixed $\beta \in \mathbb{R}^{+}, \lambda \in \mathbb{R}_{0}^{+}, \mathbf{A} \in \mathbf{C}_{0}^{\infty}, \varepsilon \in \mathbb{R}^{+}$ and $v \in(0, \beta / 2)$, the family

$$
\left\{\left(s_{1}, s_{2}\right) \mapsto \mathbf{Y}_{l, v, \varepsilon, 0}^{(\omega)}\left(s_{1}, s_{2}\right)\right\}_{l \in \mathbb{R}^{+}, \omega \in \Omega}
$$

of maps from $\mathbb{R}^{2}$ to $\mathbb{C}$ is equicontinuous, see (128). This property immediately follows from Theorem 5.1 (iii). We omit the details.

Theorem 5.17 and Lemma 5.18 imply two corollaries: The first one allows us to eliminate the $\left(s_{1}, s_{2}\right)$-dependency of the measurable set $\tilde{\Omega}\left(s_{1}, s_{2}\right)$ of Theorem 5.17. The second one concerns the continuity of the function $\Gamma_{k, q}$, which is in fact related to a matrix-valued conductivity as explained after Theorem 5.21.

\section{Corollary 5.19 (Infinite volume limit of the X-integrand-II)}

Let $\beta \in \mathbb{R}^{+}$and $\lambda \in \mathbb{R}_{0}^{+}$. Then, there is a measurable subset $\tilde{\Omega} \equiv \tilde{\Omega}^{(\beta, \lambda)} \subset \Omega$ of full measure such that, for any $s_{1}, s_{2} \in \mathbb{R}, \mathbf{A} \in \mathbf{C}_{0}^{\infty}$ and $\omega \in \tilde{\Omega}$,

$$
\lim _{l \rightarrow \infty} \mathbf{X}_{l, 0}^{(\omega)}\left(s_{1}, s_{2}\right)=\mathbf{X}_{\infty}\left(s_{1}, s_{2}\right) .
$$

Proof: $\quad$ Fix $\beta \in \mathbb{R}^{+}$and $\lambda \in \mathbb{R}_{0}^{+}$. By Theorem 5.17, for any $s_{1}, s_{2} \in \mathbb{Q}$, there is a measurable subset $\hat{\Omega}\left(s_{1}, s_{2}\right) \subset \Omega$ of full measure such that (145) holds for any $\mathbf{A} \in \mathbf{C}_{0}^{\infty}$ and $\omega \in \hat{\Omega}\left(s_{1}, s_{2}\right)$. Let $\tilde{\Omega}$ be the intersection of all such subsets $\hat{\Omega}\left(s_{1}, s_{2}\right)$. Since this intersection is countable, $\tilde{\Omega}$ is measurable and has full measure. By Lemma 5.18 and the density of $\mathbb{Q}$ in $\mathbb{R}$, it follows that (145) holds true for any $s_{1}, s_{2} \in \mathbb{R}, \mathbf{A} \in \mathbf{C}_{0}^{\infty}$ and $\omega \in \tilde{\Omega}$. 


\section{Corollary 5.20 (Continuity of paramagnetic production coefficients)}

Let $\beta \in \mathbb{R}^{+}, \lambda \in \mathbb{R}_{0}^{+}$and $k, q \in\{1,-1, \ldots, d,-d\}$. Then, the function $\Gamma_{k, q}$ from $\mathbb{R}$ to $\mathbb{C}$ defined by (140) is continuous.

Proof: $\quad$ For each $k, q \in\{1,-1, \ldots, d,-d\}$ and $t \in \mathbb{R}$, choose $\mathbf{A} \in \mathbf{C}_{0}^{\infty}$ such that, in a fixed neighborhood of $t$, the map $s \mapsto E_{\mathbf{A}}(s, x)$ is constant for any $x \in \mathbb{R}^{d}$ and

$$
\int_{\mathbb{R}^{d}} \mathrm{~d}^{d} x\left[E_{\mathbf{A}}(t, x)\right]\left(e_{q}\right)\left[E_{\mathbf{A}}(0, x)\right]\left(e_{k}\right) \neq 0 .
$$

Then, we combine the equicontinuity of the family

$$
\left\{s \mapsto \mathbf{X}_{l, 0}^{(\omega)}(s, 0)\right\}_{l \in \mathbb{R}^{+}, \omega \in \Omega}
$$

of maps from $\mathbb{R}$ to $\mathbb{C}$ given by Lemma 5.18 with Corollary 5.19 to show that the function $\Gamma_{k, q}$ is continuous at $t \in \mathbb{R}$ for each $k, q \in\{1,-1, \ldots, d,-d\}$.

Therefore, we can now use Lebesgue's dominated convergence theorem to get the energy increment (120) in the limit $\left(\eta, l^{-1}\right) \rightarrow(0,0)$ :

\section{Theorem 5.21 (Matrix-valued heat production coefficient)}

Let $\beta \in \mathbb{R}^{+}$and $\lambda \in \mathbb{R}_{0}^{+}$. Then, there is a measurable subset $\tilde{\Omega} \equiv \tilde{\Omega}^{(\beta, \lambda)} \subset \Omega$ of full measure such that, for any $\omega \in \tilde{\Omega}, \mathbf{A} \in \mathbf{C}_{0}^{\infty}$ and $t \geq t_{0}$,

$$
\mathfrak{i}_{\mathrm{p}}(t):=\lim _{\left(\eta, l^{-1}\right) \rightarrow(0,0)}\left\{\left(\eta^{2}\left|\Lambda_{l}\right|\right)^{-1} \mathfrak{I}_{\mathrm{p}}^{\left(\omega, \eta \mathbf{A}_{l}\right)}(t)\right\}=\int_{t_{0}}^{t} \mathrm{~d} s_{1} \int_{t_{0}}^{s_{1}} \mathrm{~d} s_{2} \mathbf{X}_{\infty}\left(s_{1}, s_{2}\right) .
$$

Proof: $\quad$ Recall (120), that is, for any $t \geq t_{0}$,

$$
\left(\eta^{2}\left|\Lambda_{l}\right|\right)^{-1} \mathfrak{I}_{\mathrm{p}}^{\left(\omega, \eta \mathbf{A}_{l}\right)}(t)=\int_{t_{0}}^{t} \mathrm{~d} s_{1} \int_{t_{0}}^{s_{1}} \mathrm{~d} s_{2} \mathbf{X}_{l, 0}^{(\omega)}\left(s_{1}, s_{2}\right)+\mathcal{O}(\eta)
$$

The assertion then follows from Lemma 5.18 and Corollary 5.19 together with Lebesgue's dominated convergence theorem.

Notice at this point that the theorem above together with Equation (142) means that the continuous functions $\Gamma_{k, q}$ define the entries of a matrix-valued heat production coefficient. In fact, (142) can be rewritten by using the deterministic paramagnetic transport coefficient $\boldsymbol{\Xi}_{\mathrm{p}}$ defined by (33): 


\section{Lemma $5.22\left(\Xi_{\mathrm{p}}\right.$ as heat production coefficient)}

For any $\beta \in \mathbb{R}^{+}, \lambda \in \mathbb{R}_{0}^{+}$and $s_{1}, s_{2} \in \mathbb{R}$,

$$
\mathbf{X}_{\infty}\left(s_{1}, s_{2}\right)=\int_{\mathbb{R}^{d}} \mathrm{~d}^{d} x\left\langle E_{\mathbf{A}}\left(s_{1}, x\right), \boldsymbol{\Xi}_{\mathrm{p}}\left(s_{1}-s_{2}\right) E_{\mathbf{A}}\left(s_{2}, x\right)\right\rangle .
$$

Proof: By combining [BPK2, Theorem 3.1, Lemma 5.2] with Equations (26), (29), (31), (33), (140), (142), one gets

$$
\left\{\boldsymbol{\Xi}_{\mathrm{p}}(t)\right\}_{q, k}=\left\{\boldsymbol{\Xi}_{\mathrm{p}}(t)\right\}_{k, q}=\Gamma_{k, q}(t)-\Gamma_{k, q}(0) \in \mathbb{R}
$$

for any $k, q \in\{1, \ldots, d\}$ and $t \in \mathbb{R}$. Hence,

$$
\mathbf{X}_{\infty}\left(s_{1}, s_{2}\right)=\int_{\mathbb{R}^{d}} \mathrm{~d}^{d} x \sum_{q=1}^{d}\left[E_{\mathbf{A}}\left(s_{1}, x\right)\right]\left(e_{q}\right) \sum_{k=1}^{d}\left\{\boldsymbol{\Xi}_{\mathrm{p}}\left(s_{1}-s_{2}\right)\right\}_{q, k}\left[E_{\mathbf{A}}\left(s_{2}, x\right)\right]\left(e_{k}\right) .
$$

Therefore, Theorem 4.1 (p) directly results from Theorem 5.21 and Lemma 5.22 together with Fubini's theorem. In particular, $\boldsymbol{\Xi}_{\mathrm{p}}$ can also be seen as the heat production coefficient. Under the assumption that the random variables are independently and identically distributed (i.i.d.) (Section 2.1), this coefficient becomes a scalar:

\section{Lemma 5.23 (Paramagnetic transport coefficient as a scalar)}

For any $\beta \in \mathbb{R}^{+}, \lambda \in \mathbb{R}_{0}^{+}$, there is a real function $\sigma_{\mathrm{p}} \equiv \boldsymbol{\sigma}_{\mathrm{p}}^{(\beta, \lambda)}$ such that

$$
\boldsymbol{\Xi}_{\mathrm{p}}(t)=\boldsymbol{\sigma}_{\mathrm{p}}(t) \operatorname{Id}_{\mathbb{R}^{d}}, \quad t \in \mathbb{R} .
$$

Proof: Straightforward computations using the invariance of $\mathfrak{a}_{\Omega}$ under translations, reflections and permutations of axes (cf. (8)) show from (31) and (33) that at $t \in \mathbb{R}$, the coefficient $\left\{\boldsymbol{\Xi}_{\mathrm{p}}(t)\right\}_{k, q}$ vanishes for all $k, q \in\{1, \ldots, d\}$ with $k \neq q$, while, for any $k, q \in\{1, \ldots, d\}$ and $t \in \mathbb{R}$,

$$
\left\{\boldsymbol{\Xi}_{\mathrm{p}}(t)\right\}_{k, k}=\left\{\boldsymbol{\Xi}_{\mathrm{p}}(t)\right\}_{q, q}=: \boldsymbol{\sigma}_{\mathrm{p}}(t) \text {. }
$$

It remains to prove Assertions ( $\mathbf{Q})$ and $(\mathbf{P})$ of Theorem 4.1. By Equation (55), it suffices to study the potential energy density difference $\Delta_{\mathbf{P}} \equiv \Delta_{\mathbf{P}}^{(\beta, \omega, \lambda, \mathbf{A})}$ defined by

$$
\Delta_{\mathbf{P}}(t):=\lim _{\left(\eta, l^{-1}\right) \rightarrow(0,0)}\left\{\left(\eta^{2}\left|\Lambda_{l}\right|\right)^{-1}\left(\mathbf{P}^{\left(\omega, \eta \mathbf{A}_{l}\right)}(t)-\mathfrak{I}_{\mathrm{d}}^{\left(\omega, \eta \mathbf{A}_{l}\right)}(t)\right)\right\}
$$


for any $\beta \in \mathbb{R}^{+}, \omega \in \Omega, \lambda \in \mathbb{R}_{0}^{+}, \mathbf{A} \in \mathbf{C}_{0}^{\infty}$ and $t \geq t_{0}$. This analysis is done in the following theorem:

\section{Theorem 5.24 (Potential energy density difference)}

Let $\beta \in \mathbb{R}^{+}$and $\lambda \in \mathbb{R}_{0}^{+}$. Then, there is a measurable subset $\tilde{\Omega} \equiv \tilde{\Omega}^{(\beta, \lambda)} \subset \Omega$ of full measure such that, for any $\omega \in \tilde{\Omega}, \mathbf{A} \in \mathbf{C}_{0}^{\infty}$ and $t \geq t_{0}$,

$$
\Delta_{\mathbf{P}}(t)=\int_{\mathbb{R}^{d}} \mathrm{~d}^{d} x \int_{t_{0}}^{t} \mathrm{~d} s_{1} \int_{t_{0}}^{t} \mathrm{~d} s_{2}\left\langle E_{\mathbf{A}}\left(s_{1}, x\right), \boldsymbol{\Xi}_{\mathrm{p}}\left(t-s_{2}\right) E_{\mathbf{A}}\left(s_{2}, x\right)\right\rangle .
$$

Proof: $\quad$ By [BPK2, Lemmata 5.2 and 5.13], for any $\mathbf{A} \in \mathbf{C}_{0}^{\infty}$, there is $\eta_{0} \in \mathbb{R}^{+}$ such that, for all $|\eta| \in\left(0, \eta_{0}\right]$ and $l \in \mathbb{R}^{+}$,

$$
\mathbf{P}^{(\omega, \mathbf{A})}(t)-\mathfrak{I}_{\mathrm{d}}^{(\omega, \mathbf{A})}(t)=\eta^{2}\left|\Lambda_{l}\right| \int_{t_{0}}^{t} \mathrm{~d} s_{1} \int_{t_{0}}^{t} \mathrm{~d} s_{2} \hat{\mathbf{X}}_{l}^{(\omega)}\left(s_{1}, s_{2}\right)+\mathcal{O}\left(\eta^{3} l^{d}\right)
$$

uniformly for $\beta \in \mathbb{R}^{+}, \omega \in \Omega, \lambda \in \mathbb{R}_{0}^{+}$and $t \geq t_{0}$, where

$\hat{\mathbf{X}}_{l}^{(\omega)}\left(s_{1}, s_{2}\right):=\frac{1}{4\left|\Lambda_{l}\right|} \sum_{\mathbf{x}, \mathbf{y} \in \mathfrak{K}} \int_{0}^{\beta} \mathrm{d} \alpha\left(\mathfrak{C}_{t-s_{2}+i \alpha}^{(\omega)}(\mathbf{x}, \mathbf{y})-\mathfrak{C}_{i \alpha}^{(\omega)}(\mathbf{x}, \mathbf{y})\right) \mathbf{E}_{s_{1}}^{\mathbf{A}_{l}}(\mathbf{x}) \mathbf{E}_{s_{2}}^{\mathbf{A}_{l}}(\mathbf{y})$

for any $s_{1}, s_{2} \in \mathbb{R}$. The function $\hat{\mathbf{X}}_{l}^{(\omega)}$ is very similar to $\mathbf{X}_{l, 0}^{(\omega)}$. Compare indeed the last equation with (121). As a consequence, one gets the assertion exactly in the same way one proves Theorem 5.21 and Lemma 5.22. We omit the details.

\subsection{Hilbert Space of Current Fluctuations}

\subsubsection{Positive Sesquilinear Forms}

As explained in Section 3.3 the linear subspace

$$
\mathcal{I}:=\operatorname{lin}\left\{\operatorname{Im}\left(a^{*}\left(\psi_{1}\right) a\left(\psi_{2}\right)\right): \psi_{1}, \psi_{2} \in \ell^{1}(\mathfrak{L}) \subset \ell^{2}(\mathfrak{L})\right\} \subset \mathcal{U}
$$

is an invariant space of the one-parameter (Bogoliubov) group $\tau^{(\omega, \lambda)}$ for any $\omega \in$ $\Omega$ and $\lambda \in \mathbb{R}_{0}^{+}$. by

Let the random positive sesquilinear form $\langle\cdot, \cdot\rangle_{\mathcal{I}, l}^{(\omega)} \equiv\langle\cdot, \cdot\rangle_{\mathcal{I}, l}^{(\beta, \omega)}$ in $\mathcal{I}$ be defined

$$
\left\langle I, I^{\prime}\right\rangle_{\mathcal{I}, l}^{(\omega)}:=\varrho^{(\beta, \omega, \lambda)}\left(\mathbb{F}^{(l)}(I)^{*} \mathbb{F}^{(l)}\left(I^{\prime}\right)\right), \quad I, I^{\prime} \in \mathcal{I}
$$


for any $l, \beta \in \mathbb{R}^{+}, \omega \in \Omega$ and $\lambda \in \mathbb{R}_{0}^{+}$. Here, $\mathbb{F}^{(l)}$ is the fluctuation observable defined by (40), that is,

$$
\mathbb{F}^{(l)}(I):=\frac{1}{\left|\Lambda_{l}\right|^{1 / 2}} \sum_{x \in \Lambda_{l}}\left\{\chi_{x}(I)-\varrho^{(\beta, \omega, \lambda)}\left(\chi_{x}(I)\right) \mathbf{1}\right\}, \quad I \in \mathcal{I},
$$

for each $l \in \mathbb{R}^{+}$. Recall that $\chi_{x}, x \in \mathfrak{L}$, are the (space) translation automorphisms.

In the following we aim to prove Theorem 3.5. The latter says that for $\omega$ in a subset $\tilde{\Omega} \equiv \tilde{\Omega}^{(\beta, \lambda)} \subset \Omega$ of full measure, the limit $l \rightarrow \infty$ of $\langle\cdot, \cdot\rangle_{\mathcal{I}, l}^{(\omega)}$ is a positive sesquilinear form $\langle\cdot, \cdot\rangle_{\mathcal{I}} \equiv\langle\cdot, \cdot\rangle_{\mathcal{I}}^{(\beta, \lambda)}$ on $\mathcal{I}$ which does not depend on $\omega \in \tilde{\Omega}$. To prove this, it suffices to consider elements $I, I^{\prime} \in \mathcal{I}$ of the form

$$
I=\operatorname{Im}\left(a^{*}\left(\psi_{1}\right) a\left(\psi_{2}\right)\right), \quad I^{\prime}=\operatorname{Im}\left(a^{*}\left(\psi_{1}^{\prime}\right) a\left(\psi_{2}^{\prime}\right)\right),
$$

with $\psi_{1}, \psi_{2}, \psi_{1}^{\prime}, \psi_{2}^{\prime} \in \ell^{1}(\mathfrak{L})$. In this case, we have a uniform estimate given by [BPK2, Lemma 5.10]: There is a constant $D \in \mathbb{R}^{+}$such that, for any $l, \beta \in \mathbb{R}^{+}$, $\omega \in \Omega, \lambda \in \mathbb{R}_{0}^{+}$and all $\psi_{1}, \psi_{2}, \psi_{1}^{\prime}, \psi_{2}^{\prime} \in \ell^{1}(\mathfrak{L})$,

$$
\left|\left\langle\operatorname{Im}\left(a^{*}\left(\psi_{1}\right) a\left(\psi_{2}\right)\right), \operatorname{Im}\left(a^{*}\left(\psi_{1}^{\prime}\right) a\left(\psi_{2}^{\prime}\right)\right)\right\rangle_{\mathcal{I}, l}^{(\omega)}\right| \leq D\left\|\psi_{1}\right\|_{1}\left\|\psi_{2}\right\|_{1}\left\|\psi_{1}^{\prime}\right\|_{1}\left\|\psi_{2}^{\prime}\right\|_{1} .
$$

Using this we can restrict the choice of $\psi_{1}, \psi_{2}, \psi_{1}^{\prime}, \psi_{2}^{\prime}$ to some convenient dense subset of $\ell^{1}(\mathfrak{L})$ : Let

$$
\ell_{0}^{\mathbb{Q}}:=\left\{\psi \in \ell^{1}(\mathfrak{L}): \psi \text { is a }(\mathbb{Q}+i \mathbb{Q}) \text {-valued function with finite support }\right\}
$$

and observe that it is a countable and dense subset of $\ell^{1}(\mathfrak{L})$.

By countability of $\ell_{0}^{\mathbb{Q}}$, it suffices to prove, for each $\psi_{1}, \psi_{2}, \psi_{1}^{\prime}, \psi_{2}^{\prime} \in \ell_{0}^{\mathbb{Q}}$, the existence of a subset $\tilde{\Omega}_{\psi_{1}, \psi_{2}, \psi_{1}^{\prime}, \psi_{2}^{\prime}} \subset \Omega$ of full measure such that the limit

$$
\lim _{l \rightarrow \infty}\left\langle\operatorname{Im}\left(a^{*}\left(\psi_{1}\right) a\left(\psi_{2}\right)\right), \operatorname{Im}\left(a^{*}\left(\psi_{1}^{\prime}\right) a\left(\psi_{2}^{\prime}\right)\right)\right\rangle_{\mathcal{I}, l}^{(\omega)}
$$

exists and does not depend on $\omega \in \tilde{\Omega}_{\psi_{1}, \psi_{2}, \psi_{1}^{\prime}, \psi_{2}^{\prime}}$ in order to obtain a subset

$$
\tilde{\Omega}:=\bigcap_{\psi_{1}, \psi_{2}, \psi_{1}^{\prime}, \psi_{2}^{\prime} \in l_{0}^{\mathbb{Q}}} \tilde{\Omega}_{\psi_{1}, \psi_{2}, \psi_{1}^{\prime}, \psi_{2}^{\prime}} \subset \Omega
$$

of full measure with the required properties for all $\psi_{1}, \psi_{2}, \psi_{1}^{\prime}, \psi_{2}^{\prime} \in \ell_{0}^{\mathbb{Q}}$. This is performed in the following lemma: 
Lemma 5.25 (Well-definiteness of $\langle\cdot, \cdot\rangle_{\mathcal{I}}$ )

For any $\beta \in \mathbb{R}^{+}, \lambda \in \mathbb{R}_{0}^{+}$and $\psi_{1}, \psi_{2}, \psi_{1}^{\prime}, \psi_{2}^{\prime} \in \ell_{0}^{\mathbb{Q}}$, there is a measurable subset $\tilde{\Omega}_{\psi_{1}, \psi_{2}, \psi_{1}^{\prime}, \psi_{2}^{\prime}} \subset \Omega$ of full measure such that, for any $\omega \in \tilde{\Omega}_{\psi_{1}, \psi_{2}, \psi_{1}^{\prime}, \psi_{2}^{\prime}}$,

$$
\begin{aligned}
& \lim _{l \rightarrow \infty}\left\langle\operatorname{Im}\left(a^{*}\left(\psi_{1}\right) a\left(\psi_{2}\right)\right), \operatorname{Im}\left(a^{*}\left(\psi_{1}^{\prime}\right) a\left(\psi_{2}^{\prime}\right)\right)\right\rangle_{\mathcal{I}, l}^{(\omega)} \\
= & \lim _{l \rightarrow \infty} \mathbb{E}\left[\left\langle\operatorname{Im}\left(a^{*}\left(\psi_{1}\right) a\left(\psi_{2}\right)\right), \operatorname{Im}\left(a^{*}\left(\psi_{1}^{\prime}\right) a\left(\psi_{2}^{\prime}\right)\right)\right\rangle_{\mathcal{I}, l}^{(\omega)}\right] \in \mathbb{R} .
\end{aligned}
$$

Proof: $\quad$ Let $\beta \in \mathbb{R}^{+}, \lambda \in \mathbb{R}_{0}^{+}$and $\psi_{1}, \psi_{2}, \psi_{1}^{\prime}, \psi_{2}^{\prime} \in \ell_{0}^{\mathbb{Q}}$. One uses the first equation of the proof of [BPK2, Lemma 5.10] as well as [BPK2, Eq. (134)], that is all together,

$$
\begin{array}{ll}
= & \left\langle\sum_{\mathbf{x}:=\left(x^{(1)}, x^{(2)}\right), \mathbf{y}:=\left(y^{(1)}, y^{(2)}\right) \in \mathfrak{L}^{2}} \psi_{1}\left(y^{(1)}\right) \psi_{2}\left(y^{(2)}\right) \psi_{1}^{\prime}\left(x^{(1)}\right) \psi_{2}^{\prime}\left(x^{(2)}\right)\right. \\
& \times\left[\frac{1}{4\left|\Lambda_{l}\right|} \sum_{z_{1}, z_{2} \in \Lambda_{l}} \mathfrak{C}_{0}^{(\omega)}\left(\mathbf{x}+\left(z_{1}, z_{1}\right), \mathbf{y}+\left(z_{2}, z_{2}\right)\right)\right] .
\end{array}
$$

See (122) for the definition of the map $\mathfrak{C}_{t+i \alpha}^{(\omega)}$. Then, one approximates $\mathfrak{C}_{0}^{(\omega)}$ by $\mathfrak{C}_{i \alpha}^{(\omega)}$ with $\alpha \ll \beta$ in (152) by using the bounds

$$
\begin{gathered}
\left|\frac{1}{\left|\Lambda_{l}\right|} \sum_{z_{1}, z_{2} \in \Lambda_{l}} \mathfrak{C}_{0}^{(\omega)}\left(\mathbf{x}+\left(z_{1}, z_{1}\right), \mathbf{y}+\left(z_{2}, z_{2}\right)\right)-\mathfrak{C}_{i \alpha}^{(\omega)}\left(\mathbf{x}+\left(z_{1}, z_{1}\right), \mathbf{y}+\left(z_{2}, z_{2}\right)\right)\right| \\
\leq\left\|\mathfrak{C}_{i \alpha}^{(\omega)}-\mathfrak{C}_{0}^{(\omega)}\right\|_{\mathrm{op}} \leq D \alpha
\end{gathered}
$$

for sufficiently small $\alpha \in[0, \beta]$. Here, $D \in \mathbb{R}^{+}$is a finite constant only depending on $\lambda \in \mathbb{R}_{0}^{+}$. For more details, see [BPK2, Lemma 5.3, Eq. (102)]. This allows us to use Theorems 5.1 and 5.4 in order to prove the assertion. We omit the details as it is a simpler version of results proven in this paper. See for instance Theorem 5.21 .

Therefore, we define the deterministic positive sesquilinear form $\langle\cdot, \cdot\rangle_{\mathcal{I}} \equiv$ $\langle\cdot, \cdot\rangle_{\mathcal{I}}^{(\beta, \lambda)}$ in $\mathcal{I}$ to be

$$
\left\langle I, I^{\prime}\right\rangle_{\mathcal{I}}:=\lim _{l \rightarrow \infty} \mathbb{E}\left[\left\langle I, I^{\prime}\right\rangle_{\mathcal{I}, l}^{(\omega)}\right] \in \mathbb{R}, \quad I, I^{\prime} \in \mathcal{I} .
$$

By combining (150) and Lemma 5.25 we deduce the existence of this limit for all $I, I^{\prime} \in \mathcal{I}$ as well as Theorem 3.5: 


\section{Theorem 5.26 (Sesquilinear form from current fluctuations)}

Let $\beta \in \mathbb{R}^{+}$and $\lambda \in \mathbb{R}_{0}^{+}$. Then, one has:

(i) The positive sesquilinear form $\langle\cdot, \cdot\rangle_{\mathcal{I}}$ is well-defined, i.e.,

$$
\left\langle I, I^{\prime}\right\rangle_{\mathcal{I}}:=\lim _{l \rightarrow \infty} \mathbb{E}\left[\left\langle I, I^{\prime}\right\rangle_{\mathcal{I}, l}^{(\omega)}\right] \in \mathbb{R}, \quad I, I^{\prime} \in \mathcal{I} .
$$

(ii) There is a measurable subset $\tilde{\Omega} \equiv \tilde{\Omega}^{(\beta, \lambda)} \subset \Omega$ of full measure such that, for any $\omega \in \tilde{\Omega}$,

$$
\left\langle I, I^{\prime}\right\rangle_{\mathcal{I}}=\lim _{l \rightarrow \infty}\left\langle I, I^{\prime}\right\rangle_{\mathcal{I}, l}^{(\beta, \omega, \lambda)}, \quad I, I^{\prime} \in \mathcal{I}
$$

We are now in position to introduce next the Hilbert space of current fluctuations.

\subsubsection{Hilbert Space and Dynamics}

As explained in Section 3.3, the quotient $\check{\mathcal{H}}_{\mathrm{fl}}:=\mathcal{I} / \mathcal{I}_{0}$ is a pre-Hilbert space w.r.t. to the (well-defined) scalar product

$$
\left\langle[I],\left[I^{\prime}\right]\right\rangle_{\check{\mathcal{H}}_{\mathrm{fl}}}:=\left\langle I, I^{\prime}\right\rangle_{\mathcal{I}}, \quad[I],\left[I^{\prime}\right] \in \check{\mathcal{H}}_{\mathrm{fl}},
$$

where

$$
\mathcal{I}_{0}:=\left\{I \in \mathcal{I}:\langle I, I\rangle_{\mathcal{I}}=0\right\} .
$$

The completion of $\check{\mathcal{H}}_{\mathrm{fl}}$ w.r.t. the scalar product $\langle\cdot, \cdot\rangle_{\check{\mathcal{H}}_{\mathrm{f}}}$ is the Hilbert space of current fluctuations denoted by

$$
\left(\mathcal{H}_{\mathrm{fl}},\langle\cdot, \cdot\rangle_{\mathcal{H}_{\mathrm{f}}}\right)
$$

The random dynamics defined by $\tau^{(\omega, \lambda)}$ on $\mathcal{U}$ induces a unitary time evolution on $\mathcal{H}_{\mathrm{fl}}$ :

\section{Theorem 5.27 (Dynamics of current fluctuations)}

Let $\beta \in \mathbb{R}^{+}$and $\lambda \in \mathbb{R}_{0}^{+}$. Then, there is a measurable subset $\tilde{\Omega} \equiv \tilde{\Omega}^{(\beta, \lambda)} \subset \Omega$ of full measure such that, for any $\omega \in \tilde{\Omega}$, there is a unique, strongly continuous one-parameter unitary group $\left\{\mathrm{V}_{t}^{(\omega, \lambda)}\right\}_{t \in \mathbb{R}}$ on the Hilbert space $\mathcal{H}_{\mathrm{fl}}$ obeying, for any $t \in \mathbb{R}$,

$$
\mathrm{V}_{t}^{(\omega, \lambda)}([I])=\left[\tau_{t}^{(\omega, \lambda)}(I)\right], \quad[I] \in \check{\mathcal{H}}_{\mathrm{fl}} .
$$


Proof: $\quad$ By (150), Theorem 5.26 and the stationarity of the KMS state $\varrho^{(\beta, \omega, \lambda)}$ (cf. (21)), for any $\beta \in \mathbb{R}^{+}, \omega \in \Omega$ and $\lambda \in \mathbb{R}_{0}^{+}$, the one-parameter group $\tau^{(\omega, \lambda)}$ defines a unitary group $\left\{\mathrm{V}_{t}^{(\omega, \lambda)}\right\}_{t \in \mathbb{R}}$ on the Hilbert space $\left(\mathcal{H}_{\mathrm{f}},\langle\cdot, \cdot\rangle_{\mathcal{H}_{\mathrm{f}}}\right)$ as explained in the theorem: $\tau_{t}^{(\omega, \lambda)}(\mathcal{I}) \subset \mathcal{I}$ while the stationarity of $\varrho^{(\beta, \omega, \lambda)}$ implies

$$
\left\|\left[\tau_{t}^{(\omega, \lambda)}(I)\right]\right\|_{\mathcal{H}_{\mathrm{fl}}}=\|[I]\|_{\mathcal{H}_{\mathrm{fl}}}, \quad[I] \in \check{\mathcal{H}}_{\mathrm{fl}},
$$

for all $t \in \mathbb{R}$. In particular, $\tau_{t}^{(\omega, \lambda)}\left(\mathcal{I}_{0}\right) \subset \mathcal{I}_{0}$ and hence, $\left[\tau_{t}^{(\omega, \lambda)}(I)\right] \in \check{\mathcal{H}}_{\mathrm{fl}}$ depends only on the equivalence class $[I] \in \check{\mathcal{H}}_{\mathrm{fl}}$ for all $I \in \mathcal{I}$ and $t \in \mathbb{R}$. It remains to show that, for any $\beta \in \mathbb{R}^{+}, \omega \in \Omega$ and $\lambda \in \mathbb{R}_{0}^{+}, \mathrm{V}_{t}^{(\omega, \lambda)}$ is strongly continuous at $t=0$ on a dense subset of $\mathcal{H}_{\mathrm{fl}}$.

To this end, observe that, for any $[I]$ in the dense subspace $\check{\mathcal{H}}_{\mathrm{fl}}$ of $\mathcal{H}_{\mathrm{fl}}$ and any fixed $\omega \in \Omega$,

$$
\begin{aligned}
\left\|\mathrm{V}_{t}^{(\omega, \lambda)}([I])-[I]\right\|_{\mathcal{H}_{\mathrm{f}}}^{2}= & \lim _{l \rightarrow \infty} \varrho^{(\beta, \omega, \lambda)}\left(\mathbb{F}^{(l)}\left(I-\tau_{t}^{(\omega, \lambda)}(I)\right)^{*} \mathbb{F}^{(l)}(I)\right) \\
& +\lim _{l \rightarrow \infty} \varrho^{(\beta, \omega, \lambda)}\left(\mathbb{F}^{(l)}\left(I-\tau_{-t}^{(\omega, \lambda)}(I)\right)^{*} \mathbb{F}^{(l)}(I)\right) .
\end{aligned}
$$

We assume w.l.o.g. that $I=a^{*}\left(\psi_{1}\right) a\left(\psi_{2}\right)$ for some $\psi_{1}, \psi_{2} \in \ell^{1}(\mathfrak{L})$. Then, explicit computations starting from the last equality lead to

$$
\begin{aligned}
\left\|\mathrm{V}_{t}^{(\omega, \lambda)}([I])-[I]\right\|_{\mathcal{H}_{\mathrm{fl}}}^{2}= & \lim _{l \rightarrow \infty}\left\langle I_{1}^{(\omega)}(t), I\right\rangle_{\mathcal{I}, l}^{(\omega)}+\lim _{l \rightarrow \infty}\left\langle I_{2}^{(\omega)}(t), I\right\rangle_{\mathcal{I}, l}^{(\omega)} \\
& +\lim _{l \rightarrow \infty}\left\langle I_{1}^{(\omega)}(-t), I\right\rangle_{\mathcal{I}, l}^{(\omega)}+\lim _{l \rightarrow \infty}\left\langle I_{2}^{(\omega)}(-t), I\right\rangle_{\mathcal{I}, l}^{(\omega)}
\end{aligned}
$$

where, for any $\psi_{1}, \psi_{2} \in \ell^{1}(\mathfrak{L})$,

$I_{1}^{(\omega)}(t):=a^{*}\left(\psi_{1}-\mathrm{U}_{t}^{(\omega, \lambda)} \psi_{1}\right) a\left(\psi_{2}\right) \quad$ and $\quad I_{2}^{(\omega)}(t):=a^{*}\left(\mathrm{U}_{t}^{(\omega, \lambda)} \psi_{1}\right) a\left(\psi_{2}-\mathrm{U}_{t}^{(\omega, \lambda)} \psi_{2}\right)$.

Then, by using (150) together with

$$
\lim _{t \rightarrow 0}\left\|\psi_{1}-\mathrm{U}_{t}^{(\omega, \lambda)} \psi_{1}\right\|_{1}=\lim _{t \rightarrow 0}\left\|\psi_{2}-\mathrm{U}_{t}^{(\omega, \lambda)} \psi_{2}\right\|_{1}=0, \lim _{t \rightarrow 0}\left\|\mathrm{U}_{t}^{(\omega, \lambda)} \psi_{1}\right\|_{1}=\left\|\psi_{1}\right\|_{1},
$$

we infer from (155) that

$$
\lim _{t \rightarrow 0}\left\|\mathrm{~V}_{t}^{(\omega, \lambda)}([I])-[I]\right\|_{\mathcal{H}_{\mathrm{fl}}}=0
$$

for any $\beta \in \mathbb{R}^{+}, \omega \in \Omega$ and $\lambda \in \mathbb{R}_{0}^{+}$. 
Note that the strongly continuous one-parameter unitary group $\left\{\mathrm{V}_{t}^{(\omega, \lambda)}\right\}_{t \in \mathbb{R}}$ on the Hilbert space $\left(\mathcal{H}_{\mathrm{fl}},\langle\cdot, \cdot\rangle_{\mathcal{H}_{\mathrm{f}}}\right)$ is a priori depending on the parameter $\omega \in \tilde{\Omega}$, even if Equation (48) does not depend on $\omega \in \tilde{\Omega}$. In fact, one can also construct a direct integral Hilbert space to get a deterministic, strongly continuous one-parameter unitary group $\left\{\overline{\mathrm{V}}_{t}^{(\lambda)}\right\}_{t \in \mathbb{R}}$. For the interested reader, we sketch this construction in the next subsection:

\subsubsection{Averaged Initial State and Dynamics}

Note that the map $\omega \mapsto \varrho^{(\beta, \omega, \lambda)}$ is continuous w.r.t. the topology on $\Omega$ of which $\mathfrak{A}_{\Omega}$ is the Borel $\sigma$-algebra and the weak ${ }^{*}-$ topology for states. It is a consequence of a result similar to [BR2, Proposition 5.3.25.] together with the uniqueness of $\left(\tau^{(\omega, \lambda)}, \beta\right)-$ KMS states. Then, define, for any $\beta \in \mathbb{R}^{+}$and $\lambda \in \mathbb{R}_{0}^{+}$, the averaged state $\bar{\varrho}^{(\beta, \lambda)} \in \mathcal{U}^{*}$ by

$$
\bar{\varrho}^{(\beta, \lambda)}(B):=\mathbb{E}\left[\varrho^{(\beta, \omega, \lambda)}(B)\right], \quad B \in \mathcal{U} .
$$

For any $\beta \in \mathbb{R}^{+}, \lambda \in \mathbb{R}_{0}^{+}$and $\omega \in \Omega$, let $\left(\mathcal{H}^{(\omega)}, \pi^{(\omega)}, \Psi^{(\omega)}\right)$ be the GNS representation of the $\left(\tau^{(\omega, \lambda)}, \beta\right)-\mathrm{KMS}$ state $\varrho^{(\beta, \omega, \lambda)}$. The vector $\Psi^{(\omega)}$ is cyclic and the CAR $C^{*}$-algebra $\mathcal{U}$ is separable. Therefore, there is a sequence $\left\{B_{n}\right\}_{n \in \mathbb{N}} \subset \mathcal{U}$ such that the subset

$$
\left\{\pi^{(\omega)}\left(B_{n}\right) \Psi^{(\omega)}\right\}_{n \in \mathbb{N}} \subset \mathcal{H}^{(\omega)}, \quad \omega \in \Omega,
$$

is dense in $\mathcal{H}^{(\omega)}$. Moreover, the map

$$
\omega \mapsto\left\langle\pi^{(\omega)}\left(B_{n}\right) \Psi^{(\omega)}, \pi^{(\omega)}\left(B_{m}\right) \Psi^{(\omega)}\right\rangle_{\mathcal{H}^{(\omega)}}=\varrho^{(\beta, \omega, \lambda)}\left(B_{n}^{*} B_{m}\right)
$$

is bounded and measurable w.r.t. the $\sigma$-algebra $\mathfrak{A}_{\Omega}$ for all $n, m \in \mathbb{N}$. It follows that $\left\{\mathcal{H}^{(\omega)}\right\}_{\omega \in \Omega}$ is a measurable family, see [BR1, Definition 4.4.1B.]. In particular, as the probability measure $\mathfrak{a}_{\mathbf{0}}$ is a standard measure, there is a direct integral Hilbert space

$$
\overline{\mathcal{H}}:=\int_{\Omega}^{\oplus} \mathcal{H}^{(\omega)} \mathrm{d} \mathfrak{a}_{\mathbf{0}}(\omega)
$$

with scalar product

$$
\left\langle b_{1}, b_{2}\right\rangle_{\overline{\mathcal{H}}}:=\int_{\Omega}\left\langle b_{1}^{(\omega)}, b_{2}^{(\omega)}\right\rangle_{\mathcal{H}^{(\omega)}} \mathrm{d} \mathfrak{a}_{\mathbf{0}}(\omega) .
$$

Note that $\mathcal{U}$ is the inductive limit of (finite dimensional) simple $C^{*}$-algebras $\left\{\mathcal{U}_{\Lambda}\right\}_{\Lambda \in \mathcal{P}_{f}(\mathfrak{L})}$, see [S, Lemma IV.1.2]. By [BR1, Corollary 2.6.19.], $\mathcal{U}$ is thus 
simple and hence, the $\left(\tau^{(\omega, \lambda)}, \beta\right)$-KMS state $\varrho^{(\beta, \omega, \lambda)}$ is faithful. In particular, $\pi^{(\omega)}$ is injective for any $\omega \in \Omega$. We define a separating vector

$$
\bar{\Psi}:=\int_{\Omega}^{\oplus} \Psi^{(\omega)} \mathrm{d} \mathfrak{a}_{\mathbf{0}}(\omega) \in \overline{\mathcal{H}}
$$

and a non-degenerate and injective representation

$$
\bar{\pi}:=\int_{\Omega}^{\oplus} \pi^{(\omega)} \mathrm{d} \mathfrak{a}_{\mathbf{0}}(\omega)
$$

of the $C^{*}$-algebra $\mathcal{U}$ into the space $\mathcal{B}(\overline{\mathcal{H}})$. Then we have

$$
\bar{\varrho}^{(\beta, \lambda)}(B)=\langle\bar{\Psi}, \bar{\pi}(B) \bar{\Psi}\rangle_{\overline{\mathcal{H}}}, \quad B \in \mathcal{U} .
$$

In other words, $(\overline{\mathcal{H}}, \bar{\pi})$ is a faithful representation of the $C^{*}$-algebra $\mathcal{U}$ and $\bar{\Psi}$ is a separating vector representing the state $\bar{\varrho}^{(\beta, \lambda)}$.

Observe that the one-parameter group $\tau^{(\omega, \lambda)}$ has a unique unitary representation $\left\{\mathrm{e}^{i t \mathcal{L}^{(\omega)}}\right\}_{t \in \mathbb{R}} \subset \pi^{(\omega)}(\mathcal{U})^{\prime \prime}$ with $\mathcal{L}^{(\omega)}$ being a self-adjoint operator acting on the Hilbert space $\mathcal{H}^{(\omega)}$ such that $\Psi^{(\omega)} \in \operatorname{Dom}\left(\mathcal{L}^{(\omega)}\right)$ and $\mathcal{L}^{(\omega)} \Psi^{(\omega)}=0$. The family $\left\{\mathrm{e}^{i t \mathcal{L}^{(\omega)}}\right\}_{t \in \mathbb{R}, \omega \in \Omega}$ defines a strongly continuous one-parameter unitary group $\left\{\bar{U}_{t}\right\}_{t \in \mathbb{R}}$ on $\overline{\mathcal{H}}$ by

$$
\bar{U}_{t}:=\int_{\Omega}^{\oplus} \mathrm{e}^{i t \mathcal{L}^{(\omega)}} \mathrm{d} \mathfrak{a}_{0}(\omega) .
$$

It defines an averaged unitary dynamics on $\overline{\mathcal{H}}$ which satisfies $\bar{U}_{t} \bar{\Psi}=0$. In particular we can define a deterministic one-parameter group $\bar{\tau}^{(\lambda)} \equiv\left\{\bar{\tau}_{t}^{(\lambda)}\right\}_{t \in \mathbb{R}}$ of automorphisms of $\mathcal{B}(\overline{\mathcal{H}})$ by

$$
\forall t \in \mathbb{R}, B \in \mathcal{B}(\overline{\mathcal{H}}): \quad \bar{\tau}_{t}(B):=\bar{U}_{t} B \bar{U}_{t}^{*} \in \mathcal{B}(\overline{\mathcal{H}})
$$

Using these constructions, one can perform all the arguments of Sections 5.5.1-5.5.2 by taking the invariant space

$$
\overline{\mathcal{I}}:=\int_{\Omega}^{\oplus} \pi^{(\omega)}(\mathcal{I}) \mathrm{d} \mathfrak{a}_{0}(\omega) \subset \mathcal{B}(\overline{\mathcal{H}})
$$

(cf. (147)) of the group $\bar{\tau}^{(\lambda)}$. See, e.g., Theorem 5.26 (i). Then, for any $\beta \in \mathbb{R}^{+}$ and $\lambda \in \mathbb{R}_{0}^{+}$, one obtains the existence of a unique, strongly continuous oneparameter deterministic unitary group $\left\{\overline{\mathrm{V}}_{t}^{(\lambda)}\right\}_{t \in \mathbb{R}}$ on the Hilbert space constructed 
from the space of equivalence classes $\overline{\mathcal{I}} / \overline{\mathcal{I}}_{0}$ and denoted again by $\left(\mathcal{H}_{\mathrm{f}},\langle\cdot, \cdot\rangle_{\mathcal{H}_{\mathrm{fl}}}\right)$. The unitary group $\left\{\overline{\mathrm{V}}_{t}^{(\lambda)}\right\}_{t \in \mathbb{R}}$ obeys, for any $t \in \mathbb{R}$,

$$
\overline{\mathrm{V}}_{t}^{(\lambda)}([\bar{\pi}(I)])=\left[\bar{\tau}_{t}(\bar{\pi}(I))\right], \quad I \in \mathcal{I} .
$$

Moreover, by Theorems 3.1 (p) and 5.26 (i),

$$
\left\{\boldsymbol{\Xi}_{\mathrm{p}}(t)\right\}_{k, q}=2 \operatorname{Im}\left\langle\left[\bar{\pi}\left(I_{e_{k}, 0}\right)\right], \int_{0}^{t} \overline{\mathrm{V}}_{s}^{(\lambda)}\left(\left[\bar{\pi}\left(I_{e_{q}, 0}\right)\right]\right) \mathrm{d} s\right\rangle_{\mathcal{H}_{\mathrm{fl}}}
$$

for any $\beta \in \mathbb{R}^{+}, \lambda \in \mathbb{R}_{0}^{+}, t \in \mathbb{R}$ and $k, q \in\{1, \ldots, d\}$.

Acknowledgments: We would like to thank Volker Bach, Horia Cornean, Abel Klein and Peter Müller for relevant references and interesting discussions as well as important hints. JBB and WdSP are also very grateful to the organizers of the Hausdorff Trimester Program entitled "Mathematical challenges of materials science and condensed matter physics" for the opportunity to work together on this project at the Hausdorff Research Institute for Mathematics in Bonn. This work has also been supported by the grant MTM2010-16843 of the Spanish "Ministerio de Ciencia e Innovación”as well as the FAPESP grant 2013/13215-5.

\section{References}

[BGKS] J.-M. Bouclet, F. Germinet, A. Klein And J.H. Schenker, Linear response theory for magnetic Schrödinger operators in disordered media, Journal of Functional Analysis 226 (2005) 301-372.

[BR1] O. BRATTELI AND D.W. RoBINSON, Operator Algebras and Quantum Statistical Mechanics, Vol. I, 2nd ed. Springer-Verlag, New York, 1996.

[BR2] O. BRATTELI AND D.W. RoBinson, Operator Algebras and Quantum Statistical Mechanics, Vol. II, 2nd ed. Springer-Verlag, New York, 1996.

[BPK1] J.-B. Bru, W. De Siqueira Pedra and C. Kurig, Heat Production of Non-Interacting Fermions Subjected to Electric Fields, to appear in Comm. Pure Appl. Math. (2014), 48 pages.

[BPK2] J.-B. Bru, W. De Siqueira Pedra and C. Kurig, Microscopic Conductivity Distributions of Non-Interacting Fermions, Preprint (2013). 
[CL] R. CARMona AND J. LACROIX, Spectral Theory of Random Schrödinger Operators, Birkhäuser, 1990.

[GV] G.F. Giuliani And G. Vignale, Quantum Theory of the Electron Liquid, Cambrigde Univ. Press. 2005.

[GVV] D. Goderis, A. Verbeure, And P. Vets, Non-commutative Central Limits, Probab. Th. Re1. Fields. 82 (1989), 527-544.

[KLM] A. Klein, O. Lenoble, And P. Müller, On Mott's formula for the ac-conductivity in the Anderson model, Annals of Mathematics 166 (2007) 549-577.

[KM] A. Klein And P. Müller, The Conductivity Measure for the Anderson Model, Journal of Mathematical Physics, Analysis, Geometry 4 (2008) 128-150.

[AJP] C.-A. PILlet, Quantum Dynamical Systems, in Open Quantum Systems I: The Hamiltonian Approach, Volume 1880 of Lecture Notes in Mathematics, editors: S. Attal, A. Joye, C.-A. Pillet. Springer-Verlag, 2006.

[PW] W. Pusz And S. L. Woronowicz, Passive States and KMS States for General Quantum Systems, Commun. math. Phys. 58 (1978) 273-290.

[RS1] M. REed AND B. Simon, Methods of Modern Mathematical Physics, Vol. I: Functional Analysis, Academic Press, New York-London, 1980.

[RS2] M. REED AND B. Simon, Methods of Modern Mathematical Physics, Vol. II: Fourier Analysis, Self-Adjointness, Academic Press, New YorkLondon, 1975.

[S] B. Simon, The Statistical Mechanics of Lattice Gases, Vol I, Princeton University Press, Princeton, New Jersey, 1993. 